\title{
NEUMANN DOMAINS ON QUANTUM GRAPHS
}

\author{
LIOR ALON AND RAM BAND
}

\begin{abstract}
The Neumann points of an eigenfunction $f$ on a quantum (metric) graph are the interior zeros of $f^{\prime}$. The Neumann domains of $f$ are the sub-graphs bounded by the Neumann points. Neumann points and Neumann domains are the counterparts of the well-studied nodal points and nodal domains.

We prove some foundational results in this field: bounds on the number of Neumann points and properties of the probability distribution of this number. Two basic properties of Neumann domains are presented: the wavelength capacity and the spectral position. We state and prove bounds on those as well as key features of their probability distributions.

To rigorously investigate those probabilities, we establish the notion of random variables for quantum graphs. In particular, we provide conditions for considering spectral functions of quantum graphs as random variables with respect to the natural density on $\mathbb{N}$.
\end{abstract}

[2000]35Pxx, 57M20

\section{INTRODUCTION}

Nodal domains of Laplacian eigenfunctions form a central research area within spectral geometry. Historically, the first rigorous results in the field are by Sturm [46], Courant [26] and Pleijel [42]. Many works appeared since then, treating nodal domains on manifolds, metric graphs and discrete graphs. The nodal domain study on quantum (metric) graphs is a relatively modern topic, starting ${ }^{1}$ with [32] which provides an analogue of Courant's bound for graphs and initial results on the statistics of the nodal count. Further results came afterwards, including proofs of bounds on the nodal count [45, 1, 43, 18, 10, study of nodal statistics [5, 4], solutions of nodal inverse problems [7, 16, 34] and variational characterizations of the nodal count [22, 8].

The current paper is devoted to a closely related notion, called Neumann domains. On a metric graph, nodal domains are sub-graphs bounded by the zeros of the eigenfunction. Similarly, Neumann domains are the sub-graphs bounded by the zeros of the eigenfunction's derivative. To the best of our knowledge, this is the first work on Neumann domains on graphs ${ }^{2}$. Even on manifolds, Neumann domains are a very recent topic of research within spectral theory and is currently mentioned only in [47, 39, 14, 13, 6, 11. Partial results of the current paper were already announced in [6] which reviews the Neumann domain research on manifolds and on graphs.

The paper is structured as follows. The rest of this section provides the required preliminary definitions. Our main results are stated in Section 2. The proofs are then split between a few sections: Section 3 provides the proofs for the bounds and Sections 6 and 7 contain the proofs of the probabilistic statements. In between, there are two sections which

\footnotetext{
${ }^{1}$ Noting that the the work of Sturm [46] on the interval may also be considered as a result on the simplest metric graph.

${ }^{2}$ It is worthwhile to mention the interesting recent work on the related topic of Neumann partitions on graphs [35, 33].
} 
present and develop the tools needed for proving the probabilistic statements. Section 4 presents existing methods from the literature, whereas in Section 5 we state and prove the additional required lemmas. In particular, section 5 provides tools which ought to be useful for anyone considering random variables in the context of quantum graphs. The paper is concluded with a summary section (Section 8). Appendix A describes in detail calculations of Neumann and nodal counts of some particular graph families. Appendices $\mathrm{B}$ and $\mathrm{C}$ contain proofs to some lemmas.

1.1. Basic graph definitions and notations. Throughout this paper, the graphs we consider are connected and have finite number of edges and vertices. We denote a graph by $\Gamma$ and denote by $\mathcal{V}$ and $\mathcal{E}$ its sets of vertices and edges, correspondingly. We will always assume that these sets are non-empty and denote their cardinalities by $V:=|\mathcal{V}|>0$ and $E:=|\mathcal{E}|>0$. The graph is not necessarily simple. Namely, two vertices may be connected by more than one edge and it is also possible for an edge to connect a vertex to itself. An edge connecting a vertex to itself is called a loop.

Given a vertex $v \in \mathcal{V}$ we denote the multi-set of edges connected to $v$ by $\mathcal{E}_{v}$. We note that every loop connected to $v$ will appear twice in $\mathcal{E}_{v}$. The degree of a vertex is denoted by $\operatorname{deg}(v):=\left|\mathcal{E}_{v}\right|$. The boundary of a graph is defined to be $\partial \Gamma:=\{v \in \mathcal{V} \mid \operatorname{deg}(v)=1\}$. The rest of the vertices, $\mathcal{V} \backslash \partial \Gamma$, are called interior vertices. We denote the first Betti number of a graph by

$$
\beta:=E-V+1
$$

Formally, $\beta$ is the rank of the graph's first homology. Intuitively, $\beta$ is the number of "independent" simple closed paths in the graph. A graph with $\beta=0$ is called a tree graph.

We may further identify each edge $e_{j} \in \mathcal{E}$ with a real interval of finite length $l_{j}>0$. Such a graph whose edges are supplied with lengths is called a metric graph. We commonly put all of the graph edge lengths into a vector, $\vec{l}=\left(l_{1}, l_{2} \ldots l_{E}\right)$ and denote the sum of all its entries by $|\Gamma|:=\sum_{j=1}^{E} l_{j}$. This is also called the total length of the graph. A common assumption in this paper is that the set of edge lengths form a linear independent set over $\mathbb{Q}$. We abbreviate and call such a set rationally independent.

1.2. Standard quantum graphs . It is convenient to describe a function $f$ on a metric graph $\Gamma$ in terms of its restrictions to edges, $\left.f\right|_{e}:\left[0, l_{e}\right] \rightarrow \mathbb{C}$, for $e \in \mathcal{E}$. The following function spaces are also defined in this manner:

$$
L^{2}(\Gamma):=\oplus_{e \in \mathcal{E}} L^{2}\left(\left[0, l_{e}\right]\right), \quad H^{2}(\Gamma):=\oplus_{e \in \mathcal{E}} H^{2}\left(\left[0, l_{e}\right]\right),
$$

where $H^{2}$ denotes a Sobolev space of order two. The Laplace operator $\Delta: H^{2}(\Gamma) \rightarrow L^{2}(\Gamma)$ is defined edgewise by

$$
\Delta:\left.f\right|_{e} \mapsto-\left.\frac{d^{2}}{d x_{e}^{2}} f\right|_{e}
$$

where $x_{e} \in\left[0, l_{e}\right]$ is a coordinate chosen along the edge $e$. In order for the Laplacian to be self-adjoint, its domain is restricted to functions in $H^{2}(\Gamma)$ that satisfy certain vertex conditions. A description of all vertex conditions for which the Laplacian is self-adjoint can be found for example in [20]. Throughout this paper we only consider the Neumann vertex conditions (for which the Laplacian is indeed self-adjoint). A function $f \in H^{2}(\Gamma)$ is said to satisfy Neumann vertex conditions (also known as Kirchhoff or standard conditions) at a vertex $v \in \mathcal{V}$ if 
(1) The function $f$ is continuous at $v \in \mathcal{V}$, i.e.,

$$
\forall e_{1},\left.e_{2} \in \mathcal{E}_{v} \quad f\right|_{e_{1}}(v)=\left.f\right|_{e_{2}}(v) .
$$

(2) The outgoing derivatives of $f$ at $v$, denoted by $\partial_{e} f(v)$ for every $e \in \mathcal{E}_{v}$, satisfy

$$
\sum_{e \in \mathcal{E}_{v}} \partial_{e} f(v)=0
$$

A degree two vertex with Neumann conditions may be eliminated without changing the graph's spectral properties (see [19, ex. 2.2],[15, q. 2,(e)]). This allows to assume that the graph has no vertices of degree two, which we indeed assume throughout this paper.

Definition 1.1. A standard quantum graph $\Gamma$ is a connected metric graph, with finitely many vertices and edges, and no vertices of degree two. This metric graph is equipped with the Laplace operator and Neumann vertex conditions at all vertices.

Remark 1.2. This definition excludes the case of a single loop graph. In such case all eigenvalues are degenerate and so non of the results in this paper applies to the loop graph.

If $\Gamma$ is a standard quantum graph then the corresponding Laplacian is self-adjoint with real discrete spectrum, which we order increasingly, as follows

$$
0=\lambda_{0}<\lambda_{1} \leq \lambda_{2} \nearrow \infty
$$

noting that each eigenvalue in this sequence appears as many times as its multiplicity. There exists a choice of a real orthonormal $L^{2}(\Gamma)$ basis of eigenfunctions $\left\{f_{n}\right\}_{n=0}^{\infty}$ corresponding to the eigenvalues sequence [20]. The choice of this basis may not be unique (if there are non-simple eigenvalues) but the results in this paper hold for any choice of basis. Note that the first index is zero, so that $f_{0}$ is the constant eigenfunction which corresponds to the eigenvalue $\lambda_{0}=0$ (which is a simple eigenvalue, as we assume $\Gamma$ is connected). For convenience, instead of the eigenvalues themselves, we consider their square roots, $k_{n}:=\sqrt{\lambda_{n}}$. Further information on the fundamental theory of quantum graphs may be found in [20, 30].

1.3. Loop-eigenfunctions and generic eigenfunctions. Let $\Gamma$ be a standard graph. An eigenfunction which is supported on a single loop and vanishes elsewhere on the graph is called a loop-eigenfunction. Explicitly, a function $f$ is a loop-eigenfunction supported on the loop $e$ if and only if

$$
k \in \frac{2 \pi}{l_{e}} \mathbb{N} \quad \text { and }\left.\quad f\right|_{e}(x)=A \sin (k x),\left.\quad f\right|_{\Gamma \backslash e} \equiv 0,
$$

for some $A \in \mathbb{C}$ and arc-length parametrization $x \in\left[0, l_{e}\right]$. In particular if a graph has loops then each of the loops has infinitely many loop-eigenfunctions supported on it.

Definition 1.3. Let $\Gamma$ be a standard graph. Let $f$ be an eigenfunction of $\Gamma$. We call $f$ a generic eigenfunction if it satisfies all of the following.

(1) It corresponds to a simple eigenvalue.

(2) It does not vanish at vertices, $\forall v \in \mathcal{V} f(v) \neq 0$.

(3) None of the outgoing derivatives vanish at interior vertices, $\forall v \in \mathcal{V} \backslash \partial \Gamma, \forall e \in \mathcal{E}_{v} \quad \partial_{e} f(v) \neq 0$. 
Remark 1.4. It is shown in [20, Corollary 3.1.9] that if $\Gamma$ is a tree, then any eigenfunction $f$ satisfying condition (2) must correspond to a simple eigenvalue. Hence for trees its enough for an eigenfunction to satisfy both conditions (2) and (3) to be generic.

Once a certain basis of eigenfunctions, $\left\{f_{n}\right\}_{n=0}^{\infty}$ is chosen we may define the following subsets of $\mathbb{N}$ :

$$
\begin{aligned}
& \mathcal{G}:=\left\{n \in \mathbb{N}: f_{n} \text { is generic }\right\} \\
& \mathcal{L}:=\left\{n \in \mathbb{N}: f_{n} \text { is a loop-eigenfunction }\right\} .
\end{aligned}
$$

Observe that a loop-eigenfunction is not generic, so that $\mathcal{G} \cap \mathcal{L}=\emptyset$. In order to quantify how many of the eigenfunctions belong to those sets, we introduce.

\section{Definition 1.5.}

(1) Let $A \subset \mathbb{N}$ and denote $A(N):=A \cap\{1,2, \ldots N\}$ for some $N \in \mathbb{N}$. We say that $A$ has natural density $d(A)$ if the following limit exists

$$
d(A):=\lim _{N \rightarrow \infty} \frac{|A(N)|}{N} .
$$

(2) If $\mathcal{G}$ has a positive density, $d(\mathcal{G})>0$ and $A \subset \mathcal{G}$ such that $A$ has density, we define the relative density of $A$ in $\mathcal{G}$ by

$$
d_{\mathcal{G}}(A):=\lim _{N \rightarrow \infty} \frac{|A(N)|}{|\mathcal{G}(N)|}=\frac{d(A)}{d(\mathcal{G})} .
$$

The densities of $\mathcal{L}$ and $\mathcal{G}$ are given in the following theorem ${ }^{3}$.

Theorem 1.6. 2, 5] Let $\Gamma$ be a standard graph with rationally independent edge lengths. Then both $\mathcal{L}$ and $\mathcal{G}$ have natural densities, given by

$$
d(\mathcal{L})=\frac{L_{\text {loops }}}{2|\Gamma|}, \quad d(\mathcal{G})=1-d(\mathcal{L}),
$$

where $L_{\mathrm{loops}}$ is the total length of the loops of the graph.

In particular $d(\mathcal{G}) \geq \frac{1}{2}$ and almost all non-loop-eigenfunctions are generic.

The results of this paper are stated for generic eigenfunctions. Accordingly, the probabilistic statements in the paper are stated using $d_{\mathcal{G}}$, rather than the natural density, $d$ (see Section 2). The last theorem shows that $d_{\mathcal{G}}$ and $d$ differ only if the graph has loops.

\subsection{Neumann domains and Neumann count.}

Definition 1.7. Let $f$ be a generic eigenfunction of a standard graph $\Gamma$. An interior point $x \in \Gamma \backslash \mathcal{V}$ is called a nodal point if $f(x)=0$ and is called a Neumann point if $f^{\prime}(x)=0$.

Removing the nodal points of $f$ from $\Gamma$ disconnects the graph. The connected components of this new graph are called the nodal domains of $f$. Similarly, the connected components of $\Gamma$ without $f$ 's Neumann points, are called the Neumann domains of $\Gamma$ (see Figure 1.1) . If a Neumann domain is a single interval we call it a trivial Neumann domain. Hence, a Neumann domain is non-trivial if it contains some vertex of degree at least three.

The names nodal domains and Neumann domains are adopted from similar definitions for manifolds. For graphs these names might be deceiving, as a nodal domain is actually a graph (which may be considered as a sub-graph of $\Gamma$ ), and similarly for a Neumann domain.

\footnotetext{
${ }^{3}$ This Theorem generalizes [5, Proposition A.1] and [21, Theorem 3.6], as is proven and discussed in [2].
} 


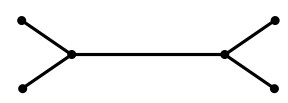

(i)

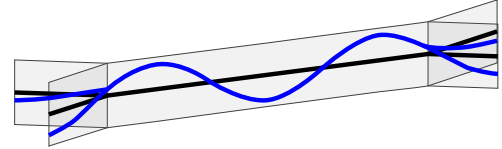

(ii)

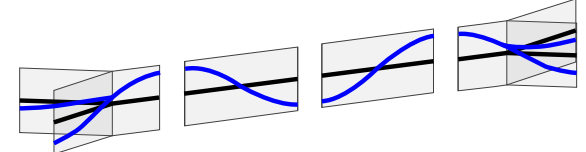

(iii)

Figure 1.1. (i) A graph $\Gamma$ (ii) An eigenfunction $f$ of $\Gamma$ (iii) A decomposition of $\Gamma$ into four Neumann domains according to $f$. The middle two Neumann domain are trivial Neumann domains.

If $f$ is a generic eigenfunction then it has a finite number of nodal points and a finite number of Neumann points and we denote those numbers by

$$
\begin{aligned}
& \phi(f):=|\{x \in \Gamma \backslash \mathcal{V} \mid f(x)=0\}|, \\
& \xi(f):=\left|\left\{x \in \Gamma \backslash \mathcal{V} \mid f^{\prime}(x)=0\right\}\right|,
\end{aligned}
$$

and correspondingly name them the nodal count and the Neumann count of $f$ ( $\phi$ stands for sign $\boldsymbol{f}$ lips of $f$, and $\xi$ stands for extremal points of $f$ ). The nodal count is well-studied both for graphs and manifolds. A fundamental result $\left(\operatorname{see}^{4}[32\right.$, 18, 9]) is the following bounds for the nodal count of the $n^{\text {th }}$ eigenfunction, $f_{n}$,

$$
0 \leq \phi\left(f_{n}\right)-n \leq \beta,
$$

where $\beta$ is the graph's first Betti number, (1.1). It is common to denote $\sigma(n):=\phi\left(f_{n}\right)-n$ and name this by nodal surplus. Analogously, denote

$$
\omega(n):=\xi\left(f_{n}\right)-n,
$$

and call $\omega(n)$ the Neumann surplus. Note that the name surplus might be misleading in this case, as possibly $\xi\left(f_{n}\right)<n$ (see Theorem 2.2).

We note that the functions $\sigma$ and $\omega$ are defined not for every natural number, but only for every $n \in \mathcal{G}$.

\subsection{Spectral position and wavelength capacity.}

Spectral position. The restriction of an eigenfunction to one of its nodal domains is an eigenfunction of that domain with Dirichlet boundary conditions. Furthermore, a well known (and commonly used) observation is that the restricted eigenfunction is the first Dirichlet eigenfunction (ground state) of that domain. This statement holds for manifolds, as well as for graphs.

It is natural to inquire whether an analogous result holds for Neumann domains. Namely, whether the restriction of an eigenfunction to a Neumann domain is a Neumann eigenfunction of that domain; and whether it is the first non-trivial (i.e., non constant) Neumann eigenfunction. The quick (and somewhat superficial) answer is that for manifolds, generically, the restricted eigenfunction is indeed a Neumann eigenfunction, but it is not necessarily the first non-trivial Neumann eigenfunction [14, 13, 11, 12]. In the current paper we treat this problem for quantum graphs. Given a generic eigenfunction $f$ with eigenvalue $k^{2}$ and a Neumann domain $\Omega$ of $f$, it is easy to show that $\left.f\right|_{\Omega}$ is an eigenfunction with eigenvalue $k^{2}$ of $\Omega$, considered as a standard graph (just observe that the vertex

\footnotetext{
${ }^{4}$ To avoid confusion when comparing to those works, recall that we start indexing the eigenvalues from $n=0$.
} 
conditions are satisfied). The interesting question would be what is the position of $k^{2}$ in the spectrum of $\Omega$. To fix terminology, we introduce

\section{Definition 1.8.}

Let $f$ be a generic eigenfunction with eigenvalue $k^{2}$ and let $\Omega$ be a Neumann domain of $f$. We define the spectral position of $\Omega$ as

$$
N(\Omega):=\mid\left\{0 \leq \lambda<k^{2} \mid \lambda \text { is an eigenvalue of } \Omega\right\} \mid,
$$

where $\Omega$ is considered as a standard graph. This notation does not explicitly include $k$, which is assumed to be understood from the context.

Wavelength capacity.

\section{Definition 1.9.}

Let $f$ be a generic eigenfunction with eigenvalue $k^{2}$ and let $\Omega$ be a Neumann domain of $f$. We define the wavelength capacity of $\Omega$ as

$$
\rho(\Omega):=\frac{|\Omega| k}{\pi}
$$

where $|\Omega|$ is the sum of edge lengths of $\Omega$. This notation of the wavelength capacity does not explicitly include $k$. It is assumed that the value of $k$ is understood from the context.

The meaning of the definition is easily demonstrated when $\Omega$ is an interval. Then, $\rho(\Omega)$ counts the number of oscillations of an eigenfunction $f$ of $\Omega$ which corresponds to the eigenvalue $k^{2}$. In other words, $\rho(\Omega)$ counts the number of 'half-wavelengths' of $f$ within $\Omega$; hence, its name.

We further note that the wavelength capacity is the one-dimensional analogue of a similar parameter for Neumann domains on manifolds. The normalized area to perimeter

ratio of a Neumann domain $\Omega$ on a two-dimensional manifold is defined to be $\frac{|\Omega| \sqrt{\lambda}}{|\partial \Omega|}$, where $|\Omega|$ is the area of the Neumann domain and $|\partial \Omega|$ is its perimeter length, [6, 28].

\section{MAin RESULTS}

Our main results concern the properties mentioned in the previous section: Neumann count, nodal count, spectral position and wavelength capacity. The Neumann count and the nodal count are properties of an eigenfunction on the whole graph, so we call those global observables. The spectral position and wavelength capacity are attributes of individual Neumann domains and they are called local observables. For each of those observables we prove bounds and basic features of their probability distributions. Denote $\mathcal{G}(N):=\mathcal{G} \cap\{0,1, \ldots N\}$. The probability distribution we assign to an observable $h: \mathcal{G} \rightarrow \mathbb{R}$ is characterized by

$$
d_{\mathcal{G}}\left(h^{-1}(B)\right):=\lim _{N \rightarrow \infty} \frac{|\{n \in \mathcal{G}(N): h(n) \in B\}|}{|\mathcal{G}(N)|} ; B \subset \mathbb{R}
$$

if such a limit exists. Indeed, it is natural to define the probability distributions of those observables in terms of the density, $d_{\mathcal{G}}$. However, $d_{\mathcal{G}}$ is not necessarily a probability measure on $\mathcal{G}$. Hence, we are required to determine whether each of the observables is a random variable with respect to $d_{\mathcal{G}}$ (the answer is not always obvious, as can be seen in the rest of this section).

Definition 2.1. A function $h: \mathcal{G} \rightarrow \mathbb{R}$ is called a random variable with respect to $d_{\mathcal{G}}$ if for every $B \subset \mathbb{R}$ Borel set, $d_{\mathcal{G}}\left(h^{-1}(B)\right)$ exists and $d_{\mathcal{G}}$ is a probability measure on the $\sigma$-algebra generated by $h$. 


\subsection{Global observables.}

Theorem 2.2. Let $\Gamma$ be a standard graph whose first Betti number is $\beta$ and boundary size is $|\partial \Gamma|$.

(1) Let $f_{n}$ be the $n$ 's eigenfunction and assume it is generic. The Neumann surplus $\omega(n):=\mu(f)-n$ is bounded by

$$
1-\beta-|\partial \Gamma| \leq \omega(n) \leq 2 \beta-1 .
$$

(2) Further assume that $\Gamma$ has rationally independent edge lengths. Then the following holds

(a) The Neumann surplus, $\omega$, is a finite random variable with respect to $d_{\mathcal{G}}$. In particular, the probability distribution of $\omega$ is

$$
\mathbb{P}(\omega=j):=d_{\mathcal{G}}\left(\omega^{-1}(j)\right) .
$$

(b) If $\omega^{-1}(j) \neq \emptyset$ then $\mathbb{P}(\omega=j)>0$.

In particular, any value which $\omega$ attains is obtained infinitely often.

(c) The probability distribution of $\omega$ is symmetric around $\frac{1}{2}(\beta-\partial \Gamma)$. Namely,

$$
\mathbb{P}(\omega=j)=\mathbb{P}(\omega=\beta-|\partial \Gamma|-j) .
$$

An interesting (and simple) observation is that the symmetry of the random variables $\omega$ and $\sigma$ implies their expected values. Namely, from the last part of Theorem 2.2 together with [5, Theorem 2.1] we deduce

Corollary 2.3. Denoting $\mathcal{G}(N):=\mathcal{G} \cap\{1, \ldots, N\}$, we have

$$
\begin{aligned}
\mathbb{E}(\sigma) & =\lim _{N \rightarrow \infty} \frac{1}{|\mathcal{G}(N)|} \sum_{n \in \mathcal{G}(N)} \sigma(n)=\frac{\beta}{2} \\
\mathbb{E}(\omega) & =\lim _{N \rightarrow \infty} \frac{1}{|\mathcal{G}(N)|} \sum_{n \in \mathcal{G}(N)} \omega(n)=\frac{\beta-|\partial \Gamma|}{2} .
\end{aligned}
$$

Thus, the expected values of the nodal surplus and of the Neumann surplus store two topological properties of the graph. We discuss the importance of this result in the context of inverse problems in Section 8.

The first part of Theorem 2.2 provides the bounds $(2.2)$ on the Neumann surplus. We conjecture that for large $\beta$ values, better bounds hold.

Conjecture 2.4. Let $f$ be a generic eigenfunction whose spectral position is $n$. The Neumann surplus $\omega(n):=\mu(f)-n$ is bounded by

$$
-1-|\partial \Gamma| \leq \omega(n) \leq \beta+1 .
$$

We note that for $\beta>2$ the bounds in (2.5) are stricter than those in (2.2). This conjecture is supported by a numerical investigation as well as analytic study of particular graph families; See Appendix A.

Theorem 2.2 sums up our knowledge on the probability distribution of the Neumann surplus for general graphs. No explicit expression for this distribution is known in general. Nevertheless, for particular graph families we do have a concrete expression.

Definition 2.5. Let $d_{1}, d_{2} \in \mathbb{N}$. A graph, each of whose vertices is either of degree $d_{1}$ or of degree $d_{2}$ is called a $\left(d_{1}, d_{2}\right)$-regular graph. 
Theorem 2.6. Let $\Gamma$ be a standard graph which is a $(3,1)$-regular tree. If $\Gamma$ has rationally independent edge lengths, then the probability distribution of the random variable $-\omega-1$ is binomial, Bin $\left(|\partial \Gamma|-2, \frac{1}{2}\right)$. Explicitly, for every integer $-|\partial \Gamma|+1 \leq j \leq-1$,

$$
\mathbb{P}(\omega=j)=\left(\begin{array}{c}
|\partial \Gamma|-2 \\
-j-1
\end{array}\right) 2^{2-|\partial \Gamma|} .
$$

This last theorem may be perceived as the Neumann domain analogue of [5, Theorem 2.3], though each of those theorems applies to completely different families of graphs. A further discussion appears in Section 8 .

\subsection{Local observables.}

Proposition 2.7. Let $\Gamma$ be a standard graph with minimal edge length $L_{\text {min }}$. Let $f_{n}$ be a generic eigenfunction which corresponds to $k_{n}>\frac{\pi}{L_{\min }}$ and let $\Omega$ be a Neumann domain of $f_{n}$. The bounds on the spectral position of $\Omega$ and its wavelength capacity are

$$
\begin{aligned}
1 & \leq N(\Omega) \leq|\partial \Omega|-1 \\
1 \leq \frac{1}{2}(N(\Omega)+1) & \leq \rho(\Omega) \leq \frac{1}{2}(N(\Omega)+|\partial \Omega|-1) \leq|\partial \Omega|-1
\end{aligned}
$$

Remark 2.8. The condition $k_{n}>\frac{\pi}{L_{\min }}$ is satisfied for almost all eigenvalues. Indeed, there

are at most $2 \frac{|\Gamma|}{L_{\min }}$ eigenvalues which do not satisfy this condition [29, Theorem 1]. This condition is needed in the proposition above in order to guarantee that $\Omega$ is a star graph (for more details, see Lemma 3.1). We also note that the lower bounds in 2.7) and (2.8) hold even without conditioning on the value of $k$ (see also the proof of the proposition).

Remark 2.9. In Appendix C we prove an analogous proposition for nodal domains.

Next, we discuss the probability distributions of the local observables $N$ and $\rho$. As implied by Proposition 2.7 those observables have non-trivial values only if the corresponding Neumann domain is non-trivial, i.e., has $|\partial \Omega|>2$, or equivalently if the corresponding Neumann domain contains an interior vertex $v \in \mathcal{V} \backslash \partial \Gamma$.

Definition 2.10. Let $f_{n}$ be a generic eigenfunction of a standard graph $\Gamma$ and let $v \in \mathcal{V} \backslash \partial \Gamma$ be an interior vertex. We denote by $\Omega_{n}^{(v)}$ the unique Neumann domain of $f_{n}$ which contains $v$. We denote the spectral position of this Neumann domain by $N^{(v)}(n):=N\left(\Omega_{n}^{(v)}\right)$ and its wavelength capacity by $\rho^{(v)}(n):=\rho\left(\Omega_{n}^{(v)}\right)$.

Proposition 2.11. Let $\Gamma$ be a standard graph with rationally independent edge lengths. Let $v \in \mathcal{V} \backslash \partial \Gamma$. Then the following hold.

(1) The spectral position, $N^{(v)}$, is a finite random variable with respect to $d_{\mathcal{G}}$. In particular, the probability distribution of $N^{(v)}$ is

$$
\mathbb{P}\left(N^{(v)}=j\right):=d_{\mathcal{G}}\left(\left(N^{(v)}\right)^{-1}(j)\right) .
$$

(2) The probability distribution of $N^{(v)}$ is symmetric around $\frac{1}{2} \operatorname{deg}(v)$. Namely,

$$
\mathbb{P}\left(N^{(v)}=j\right)=\mathbb{P}\left(N^{(v)}=\operatorname{deg}(v)-j\right) .
$$

Proposition 2.12. Let $\Gamma$ be a standard graph with rationally independent edge lengths. Let $v \in \mathcal{V} \backslash \partial \Gamma$. 
(1) There exists a discrete measure $p^{(v)}$ supported on a finite set $\left\{x_{j}\right\}_{j=1}^{m}$, and a density function $\pi^{(v)}$, such that for every interval $(a, b) \subset \mathbb{R}$

$$
d_{\mathcal{G}}\left(\left(\rho^{(v)}\right)^{-1}((a, b))\right)=\int_{a}^{b} \pi^{(v)}(x) d x+\sum_{x_{j} \in(a, b)} p^{(v)}\left(x_{j}\right) .
$$

(2) Both $\pi^{(v)}$ and $p^{(v)}$ are symmetric around $\frac{1}{2} \operatorname{deg}(v)$.

By the proposition above the pre-image $\left(\rho^{(v)}\right)^{-1}((a, b))$ has density for every interval $(a, b) \subset \mathbb{R}$. Yet, if $\pi^{(v)} \not \equiv 0$ then we prove that there exist Borel sets, $B \subset \mathbb{R}$, whose preimages $\left(\rho^{(v)}\right)^{-1}(B)$ do not have density ${ }^{5}$. In this case, $\rho^{(v)}$ is not a random variable with respect to $d_{\mathcal{G}}$. See Lemma 5.4 and Remark 5.5. Furthermore, we conjecture that $\pi^{(v)} \not \equiv 0$ and $p^{(v)} \equiv 0$, i.e., the distribution on the RHS of $(2.11)$ is absolutely continuous with no atoms and so $\rho^{(v)}$ is never a random variable with respect to $d_{\mathcal{G}}$. See further discussion after the proof of this proposition, in Remark 7.2 .

2.3. Local-global connections. We end the results section by presenting some connections between the values of local observables and those of global ones.

Proposition 2.13. Let $\Gamma$ be a standard graph with minimal edge length $L_{\text {min }}$. Let $f_{n}$ be a generic eigenfunction which corresponds to $k_{n}>\frac{\pi}{L_{\min }}$.

(1) The sum of the spectral positions of all non-trivial Neumann domains is

$$
\sum_{v \in \mathcal{V} \backslash \partial \Gamma} N\left(\Omega_{n}^{(v)}\right)=\sigma(n)-\omega(n)+(E-|\partial \Gamma|) .
$$

(2) The sum of the wavelength capacities of all non-trivial Neumann domains is

$$
\sum_{v \in \mathcal{V} \backslash \partial \Gamma} \rho\left(\Omega_{n}^{(v)}\right)=\frac{|\Gamma| k_{n}}{\pi}-(\omega(n)+n)+(E-|\partial \Gamma|) .
$$

(3) The difference of the two expressions above is

$$
\sum_{v \in \mathcal{V} \backslash \partial \Gamma}\left(N\left(\Omega_{n}^{(v)}\right)-\rho\left(\Omega_{n}^{(v)}\right)\right)=\left(n-\frac{|\Gamma| k_{n}}{\pi}\right)+\sigma(n) .
$$

Note that in the second part of the proposition, the term $\frac{|\Gamma| k_{n}}{\pi}$ may be perceived as the wavelength capacity for the whole graph (and so it is another global observable). This is also the value of the well known Weyl term, which approximates the spectral position of the eigenvalue $k_{n}$.

The last part of the proposition is an immediate implication of the first two parts. We mention it explicitly thanks to its insightful spectral meaning. The term $n-\frac{|\Gamma| k_{n}}{\pi}$ in the RHS is the difference between the actual spectral position of an eigenvalue $k_{n}$ and the Weyl term. This difference appears in the so called trace formula for quantum graphs, where it is expressed as an infinite sum of oscillatory terms corresponding to periodic orbits on the graph $([37,36])$. In the LHS of (2.14) we have a sum over the local analogues of a similar quantity, $N\left(\Omega_{n}^{(v)}\right)-\rho\left(\Omega_{n}^{(v)}\right)$. It is interesting that the deviation between the global observable $n-\frac{|\Gamma| k_{n}}{\pi}$ and the sum of the local observables, $N\left(\Omega_{n}^{(v)}\right)-\rho\left(\Omega_{n}^{(v)}\right)$, is given by the nodal surplus.

$\overline{{ }^{5} \text { Note that } \pi^{(v)}} \not \equiv 0$ is equivalent to Image $\left(\rho^{(v)}\right)$ being infinite. 


\section{Proofs of Bounds AND BASIC IDENTIties}

Proof of Theorem 2.2, (1). The main step in the proof is to show that the difference between the nodal count and the Neumann count of any generic eigenfunction $f$ on $\Gamma$ satisfies the bounds

$$
1-\beta \leq \phi(f)-\xi(f) \leq \beta-1+|\partial \Gamma| .
$$

Once we have this, the bounds $(2.2)$ follow by

(1) Observing that if $f$ is the $n$-th eigenfunction then $\phi(f)-\xi(f)=\sigma(n)-\omega(n)$.

(2) Applying the bounds for $\sigma(n)$ when $f_{n}$ is generic (see [18, Thm 2.6], [8, (1.16)]):

$$
0 \leq \sigma(n) \leq \beta
$$

Now, to prove (3.1) we start with the following decomposition

$$
\phi(f)-\xi(f)=\sum_{e \in \mathcal{E}} \phi\left(\left.f\right|_{e}\right)-\xi\left(\left.f\right|_{e}\right)
$$

where $\phi\left(\left.f\right|_{e}\right), \xi\left(\left.f\right|_{e}\right)$ are the nodal and Neumann counts on the edge $e$.

Denoting the vertices of $e$ by $u, v$ and the outgoing derivatives of $f$ at those vertices by $\partial_{e} f(v), \partial_{e} f(u)$, we show next that

$$
\phi\left(\left.f\right|_{e}\right)-\xi\left(\left.f\right|_{e}\right)=-\frac{\operatorname{sgn}\left(f(v) \partial_{e} f(v)\right)+\operatorname{sgn}\left(f(u) \partial_{e} f(u)\right)}{2},
$$

where

$$
\operatorname{sgn}(x):=\left\{\begin{array}{ll}
1 & x>0 \\
-1 & x \leq 0
\end{array} .\right.
$$

Clearly, for each $e \in \mathcal{E}$ we have $\left.f\right|_{e}(x)=A_{e} \cos \left(\varphi_{e}+k x\right)$, for some $A_{e}, \varphi_{e} \in \mathbb{R}$ and arclength parametrization $x \in\left[0, l_{e}\right]$. We continue by assuming that all edge lengths satisfy $l_{e}>\frac{2 \pi}{k}$. This assumption is justified by two observations: (a) extending the interval $\left[0, l_{e}\right]$ by $\frac{2 \pi}{k}$, while keeping $\left.f\right|_{e}(x)=A_{e} \cos \left(\varphi_{e}+k x\right)$ does not change the values and derivatives of $f$ at the endpoints of the interval (i.e., $f(u), f(v), \partial_{e} f(u), \partial_{e} f(v)$ are not changed by such an extension); (b) this extension does not change the value of $\phi\left(\left.f\right|_{e}\right)-\xi\left(\left.f\right|_{e}\right)$.

The assumption $l_{e}>\frac{2 \pi}{k}$ guarantees that there are at least two Neumann points and two nodal points on each edge. Now, examine the sets of nodal and Neumann points along the edge $e$. As the locations of these points interlace, the difference in their count can be either 0 or \pm 1 . To determine the value of this difference we only need to know whether a nodal point is the nearest to the vertex $v$ or is it a Neumann point (and similarly for the vertex $u)$.

If $v \in \mathcal{V} \backslash \partial \Gamma$ then $f(v) \partial_{e} f(v) \neq 0$ since $f$ is assumed to be generic. If $f(v) \partial_{e} f(v)>0$ then a Neumann point is closer to $v$ than any nodal point and if $f(v) \partial_{e} f(v)<0$ it is the other way around. If $v \in \partial \Gamma$ then the nearest point to $v$ is always a nodal point and by the vertex conditions which $f$ satisfies we have $f(v) \partial_{e} f(v)=0$. The arguments above yield (3.4).

Substituting (3.4) into (3.3) and changing summation to be over vertices gives

$$
\begin{aligned}
\phi(f)-\xi(f) & =-\frac{1}{2} \sum_{v \in \mathcal{V}} \sum_{e \in \mathcal{E}_{v}} \operatorname{sgn}\left(f(v) \partial_{e} f(v)\right) \\
& =\frac{|\partial \Gamma|}{2}-\frac{1}{2} \sum_{v \in \mathcal{V} \backslash \partial \Gamma} \sum_{e \in \mathcal{E}_{v}} \operatorname{sgn}\left(f(v) \partial_{e} f(v)\right),
\end{aligned}
$$


where moving to the last line, we used that $\operatorname{sgn}\left(f(v) \partial_{e} f(v)\right)=-1$ for all $v \in \partial \Gamma$. Recalling that $f$ is assumed to be generic we get that if $v \in \mathcal{V} \backslash \partial \Gamma$ then $f(v) \partial_{e} f(v) \neq 0$ for every $e \in \mathcal{E}_{v}$. But since by Neumann conditions we have $\sum_{e \in \mathcal{E}_{v}} f(v) \partial_{e} f(v)=0$, we conclude that this sum must include at least one positive term and at least one negative term, so that

$$
\forall v \in \mathcal{V} \backslash \partial \Gamma, \quad\left|\sum_{e \in \mathcal{E}_{v}} \operatorname{sgn}\left(f(v) \partial_{e} f(v)\right)\right| \leq \operatorname{deg}(v)-2 .
$$

Substituting (3.7) in (3.6) and using the identity

$$
2 E=\sum_{v \in \mathcal{V}} \operatorname{deg}(v)=|\partial \Gamma|+\sum_{v \in \mathcal{V} \backslash \partial \Gamma} \operatorname{deg}(v)
$$

gives

$$
\begin{aligned}
\left|\phi(f)-\xi(f)-\frac{|\partial \Gamma|}{2}\right| & \leq \frac{1}{2} \sum_{v \in \mathcal{V} \backslash \partial \Gamma}(\operatorname{deg}(v)-2) \\
= & \frac{1}{2}(2 E-|\partial \Gamma|)-(V-|\partial \Gamma|) \\
& =E-V+\frac{|\partial \Gamma|}{2}=\beta-1+\frac{|\partial \Gamma|}{2},
\end{aligned}
$$

which are exactly the bounds (3.1).

Before proceeding to prove Propositions 2.7 and 2.13, we bring a lemma which is used in the proofs of both propositions (and in other proofs as well).

Lemma 3.1. Let $\Gamma$ be a standard graph with minimal edge length $L_{\text {min }}$. Let $f$ be a generic eigenfunction with eigenvalue $k>\frac{\pi}{L_{\min }}$, and let $\Omega$ be a Neumann domain of $f$. Then

(1) $\Omega$ is either a star graph or an interval.

(2) $\left.f\right|_{\Omega}$ is a generic eigenfunction of $\Omega$, considered as a standard graph.

(3) The spectral position of $\Omega$ equals the nodal count of $\left.f\right|_{\Omega}$, i.e.,

$$
N(\Omega)=\phi\left(\left.f\right|_{\Omega}\right) .
$$

Proof of Proposition 3.1. The first statement of the lemma follows quite straightforwardly from the assumption $k>\frac{\pi}{L_{\min }}$. Indeed, for each edge $e \in \mathcal{E}$ we have $l_{e}>L_{\min }>\frac{\pi}{k}$ which implies that the eigenfunction $f$ has at least one Neumann point at each edge. Hence, no Neumann domain contains an entire edge of the original graph. It follows that a Neumann domain is either an interval (included in one of the graph edges) or a star graph (which contains one of the graph's interior vertices).

Next, we show that $\left.f\right|_{\Omega}$ is a generic eigenfunction of $\Omega$, where $\Omega$ is considered as a standard graph. It is clear that $\left.f\right|_{\Omega}$ is an eigenfunction of $\Omega$. In addition, $f$ does not vanish nor have vanishing derivatives at interior vertices of $\Gamma$. Hence, the same holds for $\left.f\right|_{\Omega}$, which means that $\left.f\right|_{\Omega}$ satisfies conditions (2) and (3) in the genericity definition (Definition 1.3). As mentioned in Remark 1.4, it is enough to conclude that $\left.f\right|_{\Omega}$ is generic since $\Omega$ is a tree graph.

Finally, we prove (3.11). The genericity of $\left.f\right|_{\Omega}$ together with $\Omega$ being a tree graph $(\beta=0)$ allows to apply (3.2) and conclude that the spectral position of $\left.f\right|_{\Omega}$ (which is by definition the spectral position of $\Omega$ ) equals the nodal count of $\left.f\right|_{\Omega}$, i.e., $N(\Omega)=\phi\left(\left.f\right|_{\Omega}\right)$. 
Proof of Proposition 2.13.

In the current proof we omit everywhere for brevity the subscripts ' $n$ ', using $k, f$ and $\Omega^{(v)}$ instead of $k_{n}, f_{n}$ and $\Omega_{n}^{(v)}$ as in the statement of the Proposition.

Proof of (2.12) Let $u \in \mathcal{V} \backslash \partial \Gamma$. We start by applying equation (3.6) from the proof of Theorem 2.2,(1). We apply 3.6 for the graph $\Omega^{(u)}$ and its eigenfunction $\left.f\right|_{\Omega^{(u)}}$ to get

$$
\phi\left(\left.f\right|_{\Omega^{(u)}}\right)-\xi\left(\left.f\right|_{\Omega^{(u)}}\right)=\frac{\left|\partial \Omega^{(u)}\right|}{2}-\frac{1}{2} \sum_{e \in \mathcal{E}_{u}} \operatorname{sgn}\left(f(u) \partial_{e} f(u)\right) .
$$

By Lemma 3.1 we get that $\Omega^{(u)}$ is a star graph, so that $\left|\partial \Omega^{(u)}\right|=\operatorname{deg}(u)$. Lemma 3.1 also gives $\phi\left(\left.f\right|_{\Omega^{(u)}}\right)=N\left(\Omega^{(u)}\right)$. Furthermore, $\xi\left(\left.f\right|_{\Omega^{(u)}}\right)=0$, since $\Omega^{(u)}$ is a Neumann domain and does not contain interior Neumann points. Substituting all that in 3.12 gives

$$
N\left(\Omega^{(u)}\right)=\frac{\operatorname{deg}(u)}{2}-\frac{1}{2} \sum_{e \in \mathcal{E}_{u}} \operatorname{sgn}\left(f(u) \partial_{e} f(u)\right) .
$$

Summing (3.13) over all $u \in \mathcal{V} \backslash \partial \Gamma$ and using (3.6) again yields

$$
\sum_{u \in \mathcal{V} \backslash \partial \Gamma} N\left(\Omega^{(u)}\right)=\sum_{u \in \mathcal{V} \backslash \partial \Gamma} \frac{\operatorname{deg}(u)}{2}+\phi(f)-\xi(f)-\frac{|\partial \Gamma|}{2} .
$$

Applying identity (3.8) gives

$$
\sum_{u \in \mathcal{V} \backslash \partial \Gamma} N\left(\Omega^{(u)}\right)=\phi(f)-\xi(f)+E-|\partial \Gamma|,
$$

which proves 2.12 since $\sigma(n)-\omega(n)=\phi(f)-\xi(f)$.

Proof of $(2.13)$

We denote by $\mathcal{W}$ the set of all trivial Neumann domains of $f$. Those are the Neumann domains which are intervals and do not contain any interior vertex of the graph. We have that

$$
\begin{aligned}
\frac{|\Gamma| k}{\pi} & =\sum_{v \in \mathcal{V} \backslash \partial \Gamma} \rho\left(\Omega^{(u)}\right)+\sum_{\Omega \in \mathcal{W}} \rho(\Omega) \\
& =\sum_{v \in \mathcal{V} \backslash \partial \Gamma} \rho\left(\Omega^{(u)}\right)+|\mathcal{W}|,
\end{aligned}
$$

where the first equality follows since the Neumann domains form a partition of the graph $\Gamma$ and the second equality follows since for all $\Omega \in \mathcal{W}, \rho(\Omega)=\frac{|\Omega|}{\pi} k=1$. To complete the proof we express $|\mathcal{W}|$ by using the following counting argument. Each Neumann domain in $\mathcal{W}$ has two boundary points. Each non-trivial Neumann domain is a star graph (Lemma 3.1) with $\operatorname{deg}(v)$ boundary points ( $v$ being the central vertex of the star). So, counting the boundary points of all Neumann domains of $\Gamma$ (either trivial or non-trivial) gives $2|\mathcal{W}|+\sum_{v \in \mathcal{V} \backslash \partial \Gamma} \operatorname{deg}(v)$. On the other hand, in this sum each Neumann point appears twice and each boundary point of the graph appears once,

$$
2|\mathcal{W}|+\sum_{v \in \mathcal{V} \backslash \partial \Gamma} \operatorname{deg}(v)=2 \xi(f)+|\partial \Gamma|
$$


Substituting (3.17), (3.8) and $\omega(n)=\xi(f)-n$ in (3.16) gives the required (2.13).

Proof of Proposition 2.7. Employing Lemma 3.1 the bounds we need to prove in (2.7) are equivalent to

$$
1 \leq \phi\left(\left.f\right|_{\Omega}\right) \leq|\partial \Omega|-1
$$

These bounds follow immediately from 3.1 , when taking $\Gamma=\Omega$ and noting that $\xi\left(\left.f\right|_{\Omega}\right)=$ 0 (no Neumann points within a Neumann domain) and that $\Omega$ is a star graph, so its first Betti number is $\beta=0$. Let us only remark that the lower bound in $(2.7)$ is trivial since $N(\Omega) \geq 1$ by definition. Hence, the lower bound holds even without the condition $k>\frac{\pi}{L_{\min }}$ (as is also mentioned in Remark 2.8).

Next we prove the bounds in (2.8). Clearly, the external bounds follow immediately from (2.7) and we only need to prove the internal bounds,

$$
\frac{1}{2}(N(\Omega)+1) \leq \rho(\Omega) \leq \frac{1}{2}(N(\Omega)+|\partial \Omega|-1) .
$$

The lower bound follows by applying [29, Theorem 1]. With our notations, the statement of [29, Theorem 1] is $k \geq \frac{\pi}{2|\Omega|}(N+1)$. From here, the required lower bound in $(3.19)$ follows, as $\rho=\frac{|\Omega| k}{\pi}$. We note just as above that this lower bound holds without assuming $k>\frac{\pi}{L_{\min }}$ (as is also mentioned in Remark 2.8).

We proceed to prove the upper bound in (3.19). It follows from the next lemma, whose proof is given after the proof of the proposition.

Lemma 3.2. Let $\Omega$ be a standard star graph and $f$ be a generic eigenfunction of $\Omega$ with eigenvalue $k$. Assume that $f$ has no Neumann points. Then there exists a dual standard star graph, $\widetilde{\Omega}$, which satisfies

(1) Both star graphs have the same number of edges, i.e., $|\partial \Omega|=|\partial \widetilde{\Omega}|$.

(2) There exists a generic eigenfunction $\tilde{f}$ of $\widetilde{\Omega}$ with eigenvalue $k$.

(3) The eigenfunction $\tilde{f}$ has no Neumann points.

Hence, it has a single Neumann domain, which is the whole of $\widetilde{\Omega}$.

(4) The spectral positions and the wavelength capacities of both graphs obey

$$
N(\Omega)+N(\widetilde{\Omega})=|\partial \Omega| \text { and } \rho(\Omega)+\rho(\widetilde{\Omega})=|\partial \Omega|
$$

Employing Lemma 3.1, we have that $\Omega$ is star graph (or an interval, which is a particular case of a star graph) and that $\left.f\right|_{\Omega}$ is a generic eigenfunction of $\Omega$ with eigenvalue $k$. Furthermore, as $\Omega$ is a Neumann domain, $\left.f\right|_{\Omega}$ has no Neumann points. Hence, we may apply Lemma 3.2 and write

$$
\begin{aligned}
\rho(\Omega) & =|\partial \Omega|-\rho(\widetilde{\Omega}) \\
& \leq|\partial \Omega|-\frac{1}{2}(N(\widetilde{\Omega})+1) \\
& =|\partial \Omega|-\frac{1}{2}(|\partial \Omega|-N(\Omega)+1) \\
& =\frac{1}{2}(N(\Omega)+|\partial \Omega|-1),
\end{aligned}
$$


where in the first and the third lines we have used 3.20 from Lemma 3.2; and in the second line we have applied the lower bound in 3.19 for $\Omega$. As a result we get the upper bound in (3.19).

This concludes the proof of the proposition and it is left to provide a proof for Lemma 3.2, which we do next.

Proof of Lemma 3.2. The lemma is proved by providing an explicit construction of the mentioned dual star, $\widetilde{\Omega}$. We describe this construction below and it is also demonstrated in Figure 3.1 .

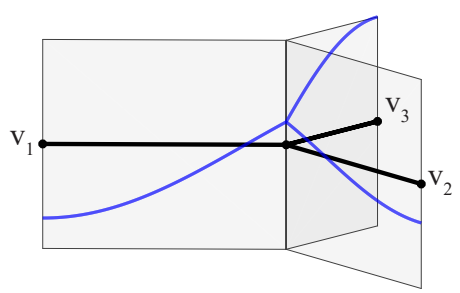

(i)

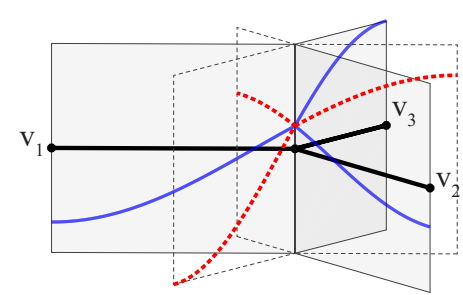

(ii)

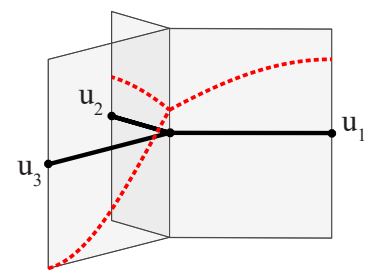

(iii)

Figure 3.1. (i) A star graph $\Omega$ with three edges (in black) and an eigenfunction $f$ (in blue) whose derivative does not vanish in the interior of $\Omega$, so that $\Omega$ is a single Neumann domain of $f$. (ii) Adding the continuation of each of the restrictions $\left.f\right|_{e_{j}}$ (in dashed red), which corresponds to $\left.\tilde{f}\right|_{\tilde{e}_{j}}$ (in the proof of Lemma 3.2 . (iii) The star graph $\widetilde{\Omega}$ (in black) which is the dual of $\Omega$ and the corresponding eigenfunction $\tilde{f}$ (in dashed red) of $\widetilde{\Omega}$.

Denote by $\left\{l_{j}\right\}_{j=1}^{|\partial \Omega|}$ the edge lengths of the star graph $\Omega$. We take $\widetilde{\Omega}$ to be a star graph with the same number of edges, $|\partial \Omega|$, and such that the edge lengths are taken to be $\tilde{l}_{j}:=\frac{\pi}{k}-l_{j}$. So, every edge of $\Omega$ has a dual edge in $\widetilde{\Omega}$. As usual, we consider $\widetilde{\Omega}$ to be a standard graph. First, to justify that the construction above defines a valid graph we need to show that $\tilde{l}_{j}>0$ for all $j$. This is equivalent to showing that $l_{j}<\frac{\pi}{k}$ for all $j$. But this is clear, since otherwise the derivative of $f$ would vanish somewhere within the edge $e_{j}$, which violates the assumption of the lemma.

Note that by construction, statement (1) of the Lemma is satisfied and we need to verify that all other statements (2), (3), (4) hold as well. To do so, note that $f$ may be written as

$$
\forall 1 \leq j \leq|\partial \Omega|,\left.\quad f\right|_{e_{j}}(x)=A_{j} \cos \left(k\left(l_{j}-x\right)\right),
$$

where $x=0$ at the central vertex, and the coefficients $A_{j}$ need to satisfy certain relations to ensure that $f$ satisfies Neumann conditions at the central vertex. Define a function $\tilde{f}$ on $\widetilde{\Omega}$ by

$$
\forall 1 \leq j \leq|\partial \Omega|,\left.\quad \tilde{f}\right|_{\tilde{e}_{j}}(x)=-A_{j} \cos \left(k\left(\tilde{l}_{j}-x\right)\right),
$$

where here as well $x=0$ at the central vertex. It is easy to see that $\tilde{f}$ satisfies Neumann condition at all the boundary vertices. At the central vertex we have

$$
\begin{aligned}
& \left.\tilde{f}\right|_{\tilde{e}_{j}}(0)=-A_{j} \cos \left(k \tilde{l}_{j}\right)=A_{j} \cos \left(k l_{j}\right)=\left.f\right|_{e_{j}}(0) \\
& \left.\tilde{f}^{\prime}\right|_{\tilde{e}_{j}}(0)=-k A_{j} \sin \left(k \tilde{l}_{j}\right)=-k A_{j} \sin \left(k l_{j}\right)=-\left.f^{\prime}\right|_{e_{j}}(0) .
\end{aligned}
$$


Since $f$ satisfies Neumann boundary conditions at the central vertex of $\Omega$ and we have the two relations above, we conclude that $\tilde{f}$ satisfies Neumann vertex conditions at the central vertex of $\widetilde{\Omega}$. Hence, $\tilde{f}$ satisfies Neumann vertex conditions at all vertices of $\widetilde{\Omega}$ and it is therefore an eigenfunction of the standard graph $\widetilde{\Omega}$ with an eigenvalue $k$. Moreover, the relations 3.23 together with the genericity of $f$ implies that $\tilde{f}$ satisfies conditions (2) and (3) of Definition 1.3 which is enough to conclude that $\tilde{f}$ is generic since $\widetilde{\Omega}$ is a tree (see Remark 1.4). This proves that statement (2) of the lemma holds.

By construction, the edge lengths of $\widetilde{\Omega}$ are bounded, $\tilde{l}_{j}<\frac{\pi}{k}$. This together with 3.22 shows that $\tilde{f}^{\prime}$ vanishes only at the graph boundary vertices, so that statement (3) of the lemma holds as well.

Finally, to show statement (4) of the lemma, we compute

$$
\rho(\widetilde{\Omega})=\sum_{j=1}^{|\partial \Omega|} \frac{k}{\pi} \tilde{l}_{j}=\sum_{j=1}^{|\partial \Omega|} \frac{k}{\pi}\left(\frac{\pi}{k}-l_{j}\right)=|\partial \Omega|-\rho(\Omega) .
$$

To compute the spectral position $N(\widetilde{\Omega})$ we observe that since $\tilde{f}$ was shown to be generic eigenfunction and since $\widetilde{\Omega}$ is a star graph with first Betti number $\beta=0$, it follows (e.g., from 3.2 ) that the spectral position $N(\widetilde{\Omega})$ equals the nodal count of $\tilde{f}$. Now, since $\tilde{l}_{j}<\frac{\pi}{k}$ and from 3.22 the number of nodal points on each edge $\tilde{e}_{j}$ is either zero (if $k \tilde{l}_{j}<\frac{\pi}{2}$ ) or one (if $k \tilde{l}_{j}>\frac{\pi}{2}$ ). The genericity of $\tilde{f}$ implies $k \tilde{l}_{j} \neq \frac{\pi}{2}$ (as otherwise $f$ would vanish at the central vertex). With those observations, we get

$$
\begin{aligned}
N(\widetilde{\Omega}) & =\left|\left\{1 \leq j \leq|\partial \Omega|: k \tilde{l}_{j}>\frac{\pi}{2}\right\}\right| \\
& =\left|\left\{1 \leq j \leq|\partial \Omega|: k l_{j}<\frac{\pi}{2}\right\}\right| \\
& =|\partial \Omega|-N(\Omega) .
\end{aligned}
$$

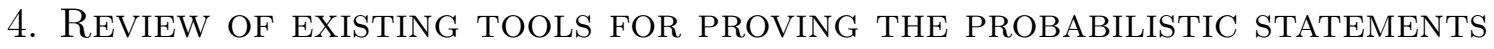

4.1. The characteristic torus and the secular manifold. Let $\Gamma$ be a standard graph with $E=|\mathcal{E}|$ edges. We will use the notation $\Gamma_{\vec{l}}$ to emphasize the dependence of $\Gamma$ on its edge lengths, $\vec{l} \in(0, \infty)^{E}$. In particular, we consider the standard graphs $\left\{\Gamma_{\vec{\kappa}}\right\}_{\vec{\kappa} \in(0,2 \pi]^{E}}$. Each with the discrete graph structure of $\Gamma$ but with edge lengths $\vec{\kappa} \in(0,2 \pi]^{E}$.

Definition 4.1. Let $\Gamma$ be a graph with $E$ edges.

(1) The flat torus $\mathbb{T}^{E}:=(\mathbb{R} / 2 \pi \mathbb{Z})^{E}$ is called the characteristic torus of $\Gamma$. We consider the coordinates of $\mathbb{T}^{E}$ as taking values in $(0,2 \pi]^{E}$. We also denote by []$: \mathbb{R}^{E} \rightarrow$ $(0,2 \pi]^{E}$ the projection to the torus by taking modulus $2 \pi$ (with a slight abuse of the usual modulus operator, as $2 \pi$ is in its image here, rather than 0 ).

(2) The following subsets of $\mathbb{T}^{E}$,

$$
\begin{gathered}
\Sigma:=\left\{\vec{\kappa} \in \mathbb{T}^{E}: 1 \text { is an eigenvalue of } \Gamma_{\vec{\kappa}}\right\} \text { and } \\
\Sigma^{\text {reg }}:=\left\{\vec{\kappa} \in \mathbb{T}^{E}: 1 \text { is a simple eigenvalue of } \Gamma_{\vec{\kappa}}\right\},
\end{gathered}
$$

are called the secular manifold and its regular part. 
We note that despite its suggestive name, $\Sigma$ is not necessarily a smooth manifold, and it may have singularities. Nevertheless, the set of its regular points is exactly $\Sigma^{\text {reg }}$, which is a real analytic manifold of dimension $E-1$, see [25, 5, 3].

The following Lemma summarizes some results from [25, 5, 3]. The proofs of all sections of this Lemma appear in [25, 5, 3] either explicitly or between the lines. Nevertheless, for completeness and didactic purpose we provide in Appendix $\mathrm{B}$ a concise proof of the Lemma.

Lemma 4.2. [25, 5, 3] Let $\Gamma$ be a standard graph.

(1) $k>0$ is a simple eigenvalue of $\Gamma_{\vec{l}}$ if and only if $[k \vec{l}] \in \Sigma^{\mathrm{reg}}$.

(2) There exists a family of functions $\left\{f_{\vec{\kappa}}\right\}_{\vec{\kappa} \in \Sigma^{\text {reg }}}$ such that

(a) For every $\vec{\kappa} \in \Sigma^{\mathrm{reg}}, f_{\vec{\kappa}}$ is an eigenfunction of $\Gamma_{\vec{\kappa}}$ corresponding to the eigenvalue 1 .

(b) For every $v, u \in \mathcal{V}$ and $e \in \mathcal{E}_{\mathcal{V}}$, there exist two real trigonometric polynomials $p_{u, v}$ and $q_{u, v, e}$ such that for every $\vec{\kappa} \in \Sigma^{\mathrm{reg}}$ :

$$
\begin{aligned}
& p_{u, v}(\vec{\kappa})=f_{\vec{\kappa}}(u) \overline{f_{\vec{\kappa}}(v)}, \text { and } \\
& q_{u, v, e}(\vec{\kappa})=f_{\vec{\kappa}}(u) \overline{\left(\partial_{e} f_{\vec{\kappa}}(v)\right)} .
\end{aligned}
$$

(3) Let $k>0$ and $\vec{l}$ such that $\vec{\kappa}:=[k \vec{l}] \in \Sigma^{\mathrm{reg}}$. Denote by $f$ the real eigenfunction of $\Gamma_{\vec{l}}$ which corresponds to the eigenvalue $k$. There exists $c \in \mathbb{C} \backslash\{0\}$ such that for every $v \in \mathcal{V}$ and $e \in \mathcal{E}_{v}$,

$$
\begin{aligned}
f(v) & =c f_{\vec{\kappa}}(v) \\
\frac{1}{k} \partial_{e} f(v) & =c \partial_{e} f_{\vec{\kappa}}(v) .
\end{aligned}
$$

Note that c may depend on $\vec{\kappa}$.

We follow the terminology of [5] and call $\left\{f_{\vec{\kappa}}\right\}_{\vec{\kappa} \in \Sigma^{\text {reg }}}$ the canonical eigenfunctions of the graph $\Gamma$.

The Lemma above emphasizes the importance of the subset $\Sigma^{\text {reg }}$ when dealing with simple eigenvalues. To specialize our discussion for generic eigenfunctions we need to define the generic manifold,

$$
\Sigma^{\text {gen }}:=\left\{\vec{\kappa} \in \Sigma^{\text {reg }} \mid f_{\vec{\kappa}} \text { is generic }\right\},
$$

the properties of which are described in the following lemma.

Lemma 4.3. [5, Thm 3.9], [2] [3, Section 5]

(1) $\Sigma^{\text {gen }}$ is a real analytic sub-manifold of $\Sigma^{\text {reg }}$ of dimension $|\mathcal{E}|-1$ and has finitely many connected components.

(2) Let $f$ be an eigenfunction of $\Gamma_{\vec{l}}$ with eigenvalue $k$. Then $f$ is generic $\Leftrightarrow \vec{\kappa}:=[k \vec{l}] \in \Sigma_{\mathcal{G}} \quad \Leftrightarrow \quad f_{\vec{\kappa}}$ is generic.

The proof of the first part of the Lemma can be found in [5, Thm 3.9], [3, Section 5] (note that our set $\Sigma^{\text {gen }}$ is different than the one in [5, Thm 3.9]; yet the proof there carries over to our case). The second part of the Lemma is a straightforward implication of (4.4), (4.5) and 4.6). 
4.2. The Barra-Gaspard measure. We start by introducing the following general framework.

Definition 4.4. [27, Definition 4.19] Let $X$ be a compact metric space and let $\mu$ be a Borel measure on $X$. A sequence $\left\{x_{n}\right\}_{n \in \mathbb{N}}$ of points in $X$ is equidistributed according to $\mu$ if for any continuous function $f \in C(X)$,

$$
\lim _{N \rightarrow \infty} \frac{1}{N} \sum_{n=1}^{N} f\left(x_{n}\right)=\int_{X} f \mathrm{~d} \mu .
$$

Lemma 4.5. [27, Exercise 4.4.2] In Definition 4.4, the continuous function $f$ may be replaced by a Riemann integrable function (i.e., a function whose discontinuity set is of $\mu$-measure zero).

Next, we specialize the discussion to graphs and their secular manifolds.

Definition 4.6. Let $\Gamma$ be a standard graph with edge lengths $\vec{l}$. Let $\left\{k_{n}\right\}_{n=1}^{\infty}$ be the multi-set of the (square root of) eigenvalues of $\Gamma$, where multiple eigenvalues appear more than once in this multi-set. The map $\varphi_{\vec{l}}: \mathcal{G} \rightarrow \mathbb{T}^{E}$ of the graph is defined as

$$
\varphi_{\vec{l}}(n):=\left[k_{n} \vec{l}\right] \text {. }
$$

By Lemma 4.3 we have that $\varphi_{\vec{l}}: \mathcal{G} \rightarrow \Sigma^{\text {gen }}$.

Definition 4.7. Define a measure $\mu_{\vec{l}}$ on the closure of the generic manifold, $\overline{\Sigma^{\text {gen }}}$ by

$$
\mathrm{d} \mu_{\vec{l}}(\vec{\kappa})= \begin{cases}C|\vec{l} \cdot \hat{n}| \mathrm{d} s & \vec{\kappa} \in \Sigma^{\text {gen }} \\ 0 & \vec{\kappa} \in \partial \Sigma^{\text {gen }},\end{cases}
$$

where $\mathrm{d} s$ is the volume element on $\Sigma^{\text {gen }}$ (which is an $E-1$ Riemannian manifold), $\hat{n}$ is the normal to $\Sigma^{\text {gen }}$, and $C=\left(\int_{\Sigma^{\text {gen }}}|\vec{l} \cdot \hat{n}| \mathrm{d} s\right)^{-1}$ is a normalization constant ${ }^{6}$.

Following [25] we call $\mu_{\vec{l}}$, the Barra-Gaspard measure.

Theorem 4.8. [17] [23, Prop. 4.4][25, Lem. 3.1] Let $\Gamma$ be a standard graph with rationally independent edge lengths $\vec{l}$. Then

(1) The sequence $\left\{\varphi_{\vec{l}}(n)\right\}_{n \in \mathcal{G}}$ is equidistributed on $\overline{\Sigma^{\mathrm{gen}}}$ with respect to the measure $\mu_{\vec{l}}$.

(2) The measure $\mu_{\vec{l}}$ is an $\vec{l}$ dependent smooth strictly positive regular Borel probability measure on $\Sigma^{\text {gen }}$.

Remark 4.9. In the references, [17] [23, Prop. 4.4][25, Lem. 3.1] similar statements to the above appear for the manifolds $\Sigma$ and $\Sigma^{\text {reg }}$, with the measure extended to the larger manifold (and normalized appropriately). Here, we restrict to $\overline{\sum^{g e n}}$ as all statements of the current paper concern generic eigenfunctions and those form a large enough venue for our explorations (see Theorem 1.6. We further note that as the sequence $\left\{\varphi_{\vec{l}}(n)\right\}_{n \in \mathcal{G}}$ is contained in $\Sigma^{\text {gen }}$ and the measure $\mu_{\vec{l}}$ is supported on $\Sigma^{\text {gen }}$, we employ the above theorem directly for $\Sigma^{\text {gen }}$ and not for its closure $\overline{\Sigma^{\text {gen }}}$.

\footnotetext{
${ }^{6}$ The normalization constant is computed explicitly in [2, 3, 5, as part of the proof of Theorem 1.6. It is given by $\frac{1}{C}=\frac{\pi}{L} \frac{1}{(2 \pi)^{E}}\left(1-\frac{L_{\text {loops }}}{2 L}\right)$ and if $\vec{l}$ is rationally independent then $\frac{1}{C}=\frac{\pi}{L} \frac{1}{(2 \pi)^{E}} d(\mathcal{G})$.
} 
Definition 4.10. We say that a set $\mathcal{A} \subset \overline{\sum^{\text {gen }}}$ has measure zero if it has zero BarraGaspard measure, $\mu_{\vec{l}}(\mathcal{A})=0$. We say that $\mathcal{A}$ is Jordan if its boundary $\partial \mathcal{A} \subset \overline{\Sigma^{\text {gen }}}$ is of measure zero.

We do not specify for which $\vec{l}$ as the above definitions are $\vec{l}$ independent. To see that observe that for any $\vec{l}, \mu_{\vec{l}}$ has a strictly positive density on $\Sigma^{\text {gen }}$ (see 4.9 ). Hence, for any measurable $\mathcal{A} \subset \overline{\bar{\Sigma}^{\text {gen }}}$,

$$
\mu_{\vec{l}}(\mathcal{A})=0 \Longleftrightarrow \int_{\mathcal{A}} \mathrm{d} s=0 .
$$

The observation that the indicator function $\chi_{\mathcal{A}}$ is Riemann integrable if and only if $\mathcal{A}$ is Jordan gives:

Corollary 4.11. Let $\Gamma$ be a standard graph with rationally independent edge lengths $\vec{l}$. Let $\mathcal{A} \subset \Sigma^{\text {gen }}$ be a Jordan set, then

$$
d_{\mathcal{G}}\left(\left\{n \in \mathcal{G}: \varphi_{\vec{l}}(n) \in \mathcal{A}\right\}\right)=\mu_{\vec{l}}(\mathcal{A}) .
$$

It is easy to demonstrate that the corollary above does not hold for sets whose boundary is not of measure zero. Take for example $\mathcal{A}=\cup_{n \in \mathcal{G}} \varphi_{l}(n)$ which violates (4.11), since $d_{\mathcal{G}}\left(\left\{n \in \mathcal{G}: \varphi_{\vec{l}}(n) \in \mathcal{A}\right\}\right)=1$, but $\mu_{\vec{l}}(\mathcal{A})=0$.

4.3. Inversion map on the secular manifold. The following lemma is useful for proving that some probability distributions are symmetric (Theorem 2.2 and Propositions 2.11 and 2.12). A proof of a similar lemma is found in [5]. Nevertheless, we provide a concise proof of the lemma in Appendix B.

Lemma 4.12. Let $\Gamma$ be a standard graph with edge lengths $\vec{l}$. Let $\mathcal{I}: \mathbb{T}^{E} \rightarrow \mathbb{T}^{E}$ be the inversion map of the torus, defined by $\mathcal{I}(\vec{\kappa})=[-\vec{\kappa}]$.

(1) Each of the manifolds, $\Sigma, \Sigma^{\mathrm{reg}}$ and $\Sigma^{\mathrm{gen}}$ is invariant under the inversion map.

(2) The restriction of the inversion to the generic part of the secular manifold, $\left.\mathcal{I}\right|_{\Sigma_{\text {gen }}}$, preserves the Barra-Gaspard measure, $\mu_{\vec{l}}$.

(3) For any $v, u \in \mathcal{V}$ and $e \in \mathcal{E}$, there are $p_{u, v}$ and $q_{u, v, e}$ which satisfy the requirements of Lemma 4.2 (2) and has the following symmetry $\backslash$ anti-symmetry relations:

$$
\begin{aligned}
p_{u, v} & \circ \mathcal{I}=p_{u, v} \\
q_{u, v, e} & \circ \mathcal{I}=-q_{u, v, e} .
\end{aligned}
$$

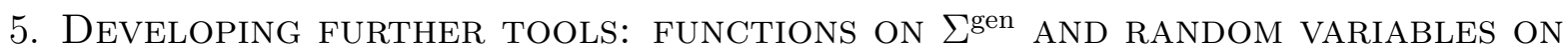
$\mathcal{G}$

This section provides the needed tools for the proofs related to the probability distributions of the observables discussed in the paper (Neumann count, spectral position, wavelength capacity). In order to do so we first relate those observables to functions on the secular manifold (Lemma 5.2). Then, we provide lemmas which aid in determining whether those observables may be considered as random variables (Lemma 5.3) or not (Lemma 5.4).

5.1. Functions on the secular manifold. The next theorem is an essential ingredient in the proofs of the main results of [5, 7].

Theorem 5.1. [5] Let $\Gamma$ be a graph with first Betti number $\beta$. There exists a function $\boldsymbol{\sigma}$ on $\Sigma^{\text {gen }}$ with the following properties: 
(1) For any choice of edge lengths $\vec{l} \in(0, \infty)^{E}$, let $\Gamma_{\vec{l}}$ be the corresponding standard graph with generic index set $\mathcal{G}$. Then for any $n \in \mathcal{G}$,

$$
\forall n \in \mathcal{G}, \quad \sigma(n)=\boldsymbol{\sigma}\left(\varphi_{\vec{l}}(n)\right) .
$$

(2) $\boldsymbol{\sigma}$ is constant on each connected component of $\Sigma^{\mathrm{gen}}$

(3) $\boldsymbol{\sigma}$ is anti-symmetric with respect to the inversion $\mathcal{I}$ in the following sense

$$
\boldsymbol{\sigma}(\mathcal{I}(\vec{\kappa}))=\beta-\boldsymbol{\sigma}(\vec{\kappa}) .
$$

The next lemma is similar in spirit to Theorem 5.1 .

Lemma 5.2. Let $\Gamma$ be a graph with first Betti number $\beta$ and let $v \in \mathcal{V} \backslash \partial \Gamma$ be an interior vertex of degree $\operatorname{deg}(v)$. There exist functions $\boldsymbol{\omega}, \boldsymbol{N}^{(\boldsymbol{v})}$ and $\boldsymbol{\rho}^{(\boldsymbol{v})}$ on $\Sigma^{\text {gen }}$ with the following properties:

(1) For any choice of edge lengths $\vec{l} \in(0, \infty)^{E}$, let $\Gamma_{\vec{l}}$ be the corresponding standard graph with generic index set $\mathcal{G}$. Then for any $n \in \mathcal{G}$,

$$
\omega(n)=\boldsymbol{\omega}\left(\varphi_{\vec{l}}(n)\right) .
$$

Moreover, denote the minimal edge length by $L_{\text {min }}$ and the sum of edge lengths by $\left|\Gamma_{\vec{l}}\right|$, and let $\left\{\Omega_{n}^{(v)}\right\}_{n \in \mathcal{G}}$ be the sequence of Neumann domains containing $v$ (see Definition 2.10). Then for any $n \in \mathcal{G}$ such that $n \geq 2 \frac{|\Gamma|}{L_{m i n}}$,

$$
\begin{gathered}
N\left(\Omega_{n}^{(v)}\right)=\boldsymbol{N}^{(\boldsymbol{v})}\left(\varphi_{\vec{l}}(n)\right), \quad \text { and } \\
\rho\left(\Omega_{n}^{(v)}\right)=\boldsymbol{\rho}^{(\boldsymbol{v})}\left(\varphi_{\vec{l}}(n)\right) .
\end{gathered}
$$

(2) The functions $\boldsymbol{\omega}$ and $\boldsymbol{N}^{(\boldsymbol{v})}$ are constant on each connected component of $\Sigma^{\text {gen }}$.

(3) $\boldsymbol{\rho}^{(\boldsymbol{v})}$ is real analytic on $\Sigma^{\text {gen }}$.

(4) $\boldsymbol{\omega}, \boldsymbol{N}^{(\boldsymbol{v})}$ and $\boldsymbol{\rho}^{(\boldsymbol{v})}$ are anti-symmetric with respect to the inversion $\mathcal{I}$ in the following sense

$$
\begin{gathered}
\boldsymbol{\omega}(\mathcal{I}(\vec{\kappa}))=\beta-|\partial \Gamma|-\boldsymbol{\omega}(\vec{\kappa}), \\
\boldsymbol{N}^{(\boldsymbol{v})}(\mathcal{I}(\vec{\kappa}))=\operatorname{deg}(v)-\boldsymbol{N}^{(\boldsymbol{v})}(\vec{\kappa}), \text { and } \\
\boldsymbol{\rho}^{(\boldsymbol{v})}(\mathcal{I}(\vec{\kappa}))=\operatorname{deg}(v)-\boldsymbol{\rho}^{(\boldsymbol{v})}(\vec{\kappa}) .
\end{gathered}
$$

Proof. The functions $p_{v, v}$ and $q_{v, v, e}$ (defined in Lemmas 4.2 and 4.12 ) are used for expressing the functions $\boldsymbol{\omega}, \boldsymbol{N}^{(\boldsymbol{v})}$ and $\boldsymbol{\rho}^{(\boldsymbol{v})}$ stated in the Lemma. Let us therefore recall some of their properties that are stated in Lemmas 4.2 and 4.12. For each $v \in \mathcal{V} \backslash \partial \Gamma$ and $e \in \mathcal{E}_{v}$, the functions $p_{v, v}$ and $q_{v, v, e}$ are real trigonometric polynomials in $\vec{\kappa}$ that do not vanish on $\Sigma^{\text {gen }}$. Their signs, $\operatorname{sgn}\left(p_{v, v}\right)$ and $\operatorname{sgn}\left(q_{v, v, e}\right)$, are therefore constant on each connected component of $\Sigma^{\text {gen }}$. Moreover, $p_{v, v}$ and $q_{v, v, e}$ are symmetric $\backslash$ anti-symmetric with respect to $\mathcal{I}$. That is,

$$
\begin{aligned}
p_{v, v} & \circ \mathcal{I}=p_{v, v}, \text { and } \\
q_{v, v, e} & \circ \mathcal{I}=-q_{v, v, e} .
\end{aligned}
$$

In addition, for any $\vec{\kappa} \in \Sigma^{\text {gen }}$, the canonical eigenfunction $f_{\vec{\kappa}}$ (i.e., the eigenfunction of $\Gamma_{\vec{\kappa}}$ with eigenvalue $k=1$ ) is generic, and satisfies:

$$
\begin{aligned}
p_{v, v}(\vec{\kappa}) & =\left|f_{\vec{\kappa}}(v)\right|^{2} \neq 0, \text { and } \\
q_{v, v, e}(\vec{\kappa}) & =f_{\vec{\kappa}}(v) \overline{\partial_{e} f_{\vec{\kappa}}(v)} \neq 0 .
\end{aligned}
$$

Another useful observation is that since both $p_{v, v}$ and $q_{v, v, e}$ are real, continuous and non vanishing on $\Sigma^{\text {gen }}$. 
The rest of the proof is divided into three parts, each treating one of the functions $\boldsymbol{\omega}$, $N^{(v)}$ and $\rho^{(v)}$.

Proof for the Neumann surplus, $\boldsymbol{\omega}$

We refer to the proof of the Theorem 2.2, (11) and employ equation (3.6) to write

$$
\begin{aligned}
\omega(n) & =\sigma(n)-\left(\phi\left(f_{n}\right)-\mu\left(f_{n}\right)\right) \\
& =\sigma(n)-\frac{|\partial \Gamma|}{2}+\frac{1}{2} \sum_{v \in \mathcal{V} \backslash \partial \Gamma} \sum_{e \in \mathcal{E}_{v}} \operatorname{sgn}\left(f_{n}(v) \overline{\partial_{e} f_{n}(v)}\right) .
\end{aligned}
$$

Now, we express all terms in the RHS of (5.13) as functions on $\Sigma^{\text {gen }}$. By Theorem 5.1 there exists a function $\boldsymbol{\sigma}$ on $\Sigma^{\text {gen }}$ such that $\sigma(n)=\boldsymbol{\sigma}\left(\varphi_{\vec{l}}(n)\right)$ for all $n \in \mathcal{G}$ and the function $\sigma$ is constant on each connected component of $\Sigma^{\text {gen }}$. In addition, by (4.3), 4.4) and 4.5) in Lemma 4.2

$$
\begin{aligned}
\forall n \in \mathcal{G}, \forall v \in \mathcal{V}, \forall e \in \mathcal{E}_{v}, \quad \frac{1}{k_{n}} f_{n}(v) \overline{\partial_{e} f_{n}(v)} & =|c|^{2} f_{\varphi_{\vec{l}}(n)}(v) \overline{\partial_{e} f_{\varphi_{\vec{l}}(n)}(v)} \\
& =|c|^{2} q_{v, v, e}\left(\varphi_{\vec{l}}(n)\right),
\end{aligned}
$$

for some $c \in \mathbb{C} \backslash\{0\}$. Hence defining

$$
\boldsymbol{\omega}(\vec{\kappa}):=\boldsymbol{\sigma}(\vec{\kappa})-\frac{|\partial \Gamma|}{2}+\frac{1}{2} \sum_{v \in \mathcal{V} \backslash \partial \Gamma} \sum_{e \in \mathcal{E}_{v}} \operatorname{sgn}\left(q_{v, v, e}(\vec{\kappa})\right),
$$

we get that for all $n \in \mathcal{G}, \omega(n)=\boldsymbol{\omega}\left(\varphi_{i}(n)\right)$, as required. Parts (2) and (4) of the Lemma (for $\boldsymbol{\omega}$ ) follow easily. Indeed, $\boldsymbol{\omega}$ is constant on each connected component of $\Sigma^{\text {gen }}$, as both $\boldsymbol{\sigma}$ and $\operatorname{sgn}\left(q_{v, v, e}\right)$ have this property. That $\boldsymbol{\omega}$ attains only finitely many values follows from (5.16) together with the statement that $\boldsymbol{\sigma}$ attains finitely many values by Theorem 5.1. In addition, the anti-symmetric property of $\boldsymbol{\omega}, 5.6$, follows immediately from the anti-symmetry of $q_{v, v, e}, 4.13$ and of $\boldsymbol{\sigma}$ (5.2.

Proof for the spectral position, $\boldsymbol{N}^{(v)}$

The $n^{\text {th }}$ eigenvalue is bounded, $k_{n} \geq \frac{\pi}{2|\Gamma|}(n+1)$, [29, Theorem 1]. Hence, assuming $n \geq 2 \frac{|\Gamma|}{L_{\min }}$ as in 5.4, we get that $k_{n}>\frac{\pi}{L_{\min }}$. This, together with $n \in \mathcal{G}$ are the required assumptions in Lemma 3.1 . That lemma guarantees that $\Omega_{n}^{(v)}$ is a star graph, that $\left.f_{n}\right|_{\Omega_{n}^{(v)}}$ is a generic eigenfunction and that $N\left(\Omega_{n}^{(v)}\right)=\phi\left(\left.f_{n}\right|_{\Omega_{n}^{(v)}}\right)$.

To do so, we employ equation 3.6 , taking the graph to be $\Omega_{n}^{(v)}$, and getting

$$
\begin{aligned}
\phi\left(\left.f_{n}\right|_{\Omega_{n}^{(v)}}\right) & =\frac{1}{2} \operatorname{deg}(v)-\frac{1}{2} \sum_{e \in \mathcal{E}_{v}} \operatorname{sgn}\left(f_{n}(v) \overline{\partial_{e} f_{n}(v)}\right) \\
& =\frac{1}{2} \operatorname{deg}(v)-\frac{1}{2} \sum_{e \in \mathcal{E}_{v}} \operatorname{sgn}\left(f_{\varphi_{\bar{l}}(n)}(v) \overline{\partial_{e} f_{\varphi_{\bar{l}}(n)}(v)}\right) \\
& =\frac{1}{2} \operatorname{deg}(v)-\frac{1}{2} \sum_{e \in \mathcal{E}_{v}} \operatorname{sgn}\left(q_{v, v, e}\left(\varphi_{\bar{l}}(n)\right)\right),
\end{aligned}
$$


where moving to the second line we used (4.4) and (4.5) in Lemma 4.2 and the last line follows from 4.3 . This motivates us to define $\boldsymbol{N}^{(\boldsymbol{v})}: \Sigma^{\mathrm{gen}} \rightarrow \mathbb{N}$ by

$$
\boldsymbol{N}^{(\boldsymbol{v})}(\vec{\kappa}):=\frac{1}{2} \operatorname{deg}(v)-\frac{1}{2} \sum_{e \in \mathcal{E}_{v}} \operatorname{sgn}\left(q_{v, v, e}(\vec{\kappa})\right) .
$$

From all the arguments above, we obtain that $N\left(\Omega_{n}^{(v)}\right)=\phi\left(\left.f_{n}\right|_{\Omega_{n}^{(v)}}\right)=\boldsymbol{N}^{(v)}\left(\varphi_{\vec{l}}(n)\right)$, for any $n \in \mathcal{G}$ such that $n \geq 2 \frac{|\Gamma|}{L_{\min }}$. Parts (2) and 4 of the Lemma (for $\boldsymbol{N}^{(\boldsymbol{v})}$ ) now follow easily. Indeed, $\boldsymbol{N}^{(\boldsymbol{v})}$ is constant on connected components of $\Sigma^{\text {gen }}$, as $\operatorname{sgn}\left(q_{v, v, e}\right)$ have this property. That $\boldsymbol{N}^{(\boldsymbol{v})}$ attains only finitely many values follows immediately from (5.20). In addition, (5.7), the anti-symmetric property of $\boldsymbol{N}^{(\boldsymbol{v})}$ follows from the anti-symmetry of $q_{v, v, e}, 4.13$.

Proof for the wavelength capacity, $\boldsymbol{\rho}^{(\boldsymbol{v})}$

Similarly to the previous part of the proof, we get that the assumption $n \in \mathcal{G}$ and $n \geq$ $2 \frac{|\Gamma|}{L_{\min }}$ in 5.5 implies that $\Omega_{n}^{(v)}$ is a star graph and that $\left.f_{n}\right|_{\Omega_{n}^{(v)}}$ is a generic eigenfunction. We denote the edges of $\Omega_{n}^{(v)}$ by $\left\{\tilde{e}_{j}\right\}_{j=1}^{\operatorname{deg}(v)}$ and the corresponding edge lengths by $\left\{\tilde{l}_{j}\right\}_{j=1}^{\operatorname{deg}(v)}$. With this notation we may write the eigenfunction $\left.f_{n}\right|_{\Omega_{n}^{(v)}}$ as

$$
\left.f_{n}\right|_{\tilde{e}_{j}}(x)=C_{j} \cos \left(k_{n}\left(\tilde{l}_{j}-x\right)\right)
$$

for some real coefficients $C_{j}$ and using the arc-length parametrization $x \in\left[0, \tilde{l}_{j}\right]$ with $x=0$ at the central vertex. The genericity of $\left.f_{n}\right|_{\Omega_{n}^{(v)}}$ implies that neither its value nor its derivative vanish at the central vertex. Since $\Omega_{n}^{(v)}$ is a Neumann domain, the derivative of $\left.f_{n}\right|_{\Omega_{n}^{(v)}}$ does not vanish at the interior of the edges, $\tilde{e}_{j}$. It vanishes only at the boundary vertices. These restrictions on values and derivatives of $\left.f_{n}\right|_{\tilde{e}_{j}}$ together with (5.21) imply that for all $j, k_{n} \tilde{l}_{j} \in\left(0, \frac{\pi}{2}\right) \cup\left(\frac{\pi}{2}, \pi\right)$. Hence, using (5.21) we write

$$
\begin{aligned}
\tan \left(k_{n} \tilde{l}_{j}\right) & =\left.\frac{\left.\left.f_{n}\right|_{\tilde{e}_{j}} \cdot \frac{1}{k_{n}} \partial_{\tilde{e}_{j}} f_{n}\right|_{\tilde{e}_{j}}}{\left.f_{n}^{2}\right|_{\tilde{e}_{j}}}\right|_{x=0} \\
& =\frac{f_{\varphi_{\vec{l}}(n)}(v) \cdot \overline{\partial_{e_{j}} f_{\varphi_{\vec{l}}(n)}(v)}}{f_{\varphi_{\vec{l}}(n)}(v) \overline{f_{\varphi_{\vec{l}}(n)}(v)}} \\
& =\frac{q_{v, v, e_{j}}\left(\varphi_{\vec{l}}(n)\right)}{p_{v, v}\left(\varphi_{\vec{l}}(n)\right)}
\end{aligned}
$$

where moving to the second line we used (4.4) and (4.5) in Lemma 4.2, and also identified the edge $\tilde{e}_{j}$ of $\Omega_{n}^{(v)}$ as a subset of an edge $e_{j}$ on the whole graph, so that $\partial_{\tilde{e}_{j}}=\partial_{e_{j}}$. The the last line in 5.22 follows from $4.2,44.3$. Next, to express $k_{n} \tilde{l}_{j}$, we use the following branch of the inverse tangent, $\tan ^{-1}: \mathbb{R} \backslash\{0\} \rightarrow\left(0, \frac{\pi}{2}\right) \cup\left(\frac{\pi}{2}, \pi\right)$, which is valid since we have shown above that $k_{n} \tilde{l}_{j} \in\left(0, \frac{\pi}{2}\right) \cup\left(\frac{\pi}{2}, \pi\right)$. Summing over all edges of the star graph 
$\Omega_{n}^{(v)}$ gives

$$
\begin{aligned}
\rho\left(\Omega_{n}^{(v)}\right) & =\frac{1}{\pi} \sum_{j=1}^{\operatorname{deg}(v)} k_{n} \tilde{l}_{j} \\
& =\frac{1}{\pi} \sum_{j=1}^{\operatorname{deg}(v)} \tan ^{-1}\left(\frac{q_{v, v, e_{j}}\left(\varphi_{\vec{l}}(n)\right)}{p_{v, v}\left(\varphi_{\vec{l}}(n)\right)}\right) .
\end{aligned}
$$

Hence, to obtain the required relation (5.5), we define the wavelength capacity function $\boldsymbol{\rho}^{(\boldsymbol{v})}: \Sigma^{\text {gen }} \rightarrow \mathbb{R}$ as

$$
\boldsymbol{\rho}^{(v)}(\vec{\kappa}):=\frac{1}{\pi} \sum_{j=1}^{\operatorname{deg}(v)} \tan ^{-1}\left(\frac{q_{v, v, e_{j}}(\vec{\kappa})}{p_{v, v}(\vec{\kappa})}\right) .
$$

Parts (3) and (4) of the Lemma (for $\rho^{(v)}$ ) now follow. To show that $\boldsymbol{\rho}^{(\boldsymbol{v})}$ is real analytic, we first notice that $q_{v, v, e_{j}}$ and $p_{v, v}$ are real trigonometric polynomials (on $\mathbb{T}^{E}$ ) and as such are real analytic on $\mathbb{T}^{E}$. With the aid of the real analytic version of the implicit function theorem [38] we get that the restrictions of $q_{v, v, e_{j}}$ and $p_{v, v}$ to the real analytic manifold $\Sigma^{\text {gen }}$ are real analytic, and so does $\frac{q_{v, v, e_{j}}}{p_{v, v}}$, as $p_{v, v}$ does not vanish on $\Sigma^{\text {gen }}$. Since $\tan ^{-1}$ is also real analytic, we get that $\boldsymbol{\rho}^{(\boldsymbol{v})}$ is real analytic on $\Sigma^{\mathrm{reg}}$.

In addition, the anti-symmetric property of $\rho^{(v)},(5.8)$, follows straightforwardly by combining the anti-symmetry of $q_{v, v, e},(5.10)$, the symmetry of $p_{v, v},(5.9)$, and the antisymmetry of the branch of $\tan ^{-1}$ we have chosen, $\tan ^{-1}(-x)=\pi-\tan ^{-1}(x)$.

5.2. Observables as random variables. This paper concerns various functions (observables) such as the nodal surplus, $\sigma$, the Neumann surplus, $\omega$, the spectral position, $N^{(v)}$ and the wavelength capacity, $\rho^{(v)}$. All those functions are defined on the generic index set, $\mathcal{G}$. In this section we provide the tools needed to show whether such a function may be considered as a random variable with respect to $d_{\mathcal{G}}$ (see Definition 2.1). To this end, we need to achieve two tasks; first present a well-defined probability space on $\mathcal{G}$ with the probability measure $d_{\mathcal{G}}$; and second, to show that the considered function is measurable. Even the first task is non-trivial, as the density $d_{\mathcal{G}}$ is not countably additive on the power set of $\mathcal{G}$ and we should carefully choose our $\sigma$-algebra on $\mathcal{G}$ to ensure this.

Lemma 5.3. Let $\Gamma$ be a standard graph with rationally independent edge lengths $\vec{l}$. Let $\boldsymbol{\alpha}: \Sigma^{\text {gen }} \rightarrow \mathbb{R}$ and $\alpha: \mathcal{G} \rightarrow \mathbb{R}$ such that

$$
\forall n \in \mathcal{G}, \quad \alpha(n):=\boldsymbol{\alpha}\left(\varphi_{\vec{l}}(n)\right) .
$$

If each element of the $\sigma$-algebra generated by $\boldsymbol{\alpha}$ is a Jordan set (i.e., having boundary of measure zero) then $\alpha$ is a random variable with respect to $d_{\mathcal{G}}$. Moreover the probability distribution of $\alpha$ is given by

$$
\forall j \in \operatorname{Image}(\alpha) d_{\mathcal{G}}\left(\alpha^{-1}(j)\right)=\mu_{\vec{l}}\left(\boldsymbol{\alpha}^{-1}(j)\right),
$$

where $\mu_{\vec{l}}$ is the Barra-Gaspard measure (4.9).

Proof. Let $\mathcal{F}_{\alpha}$ be the $\sigma$-algebra on $\mathcal{G}$ generated by $\alpha$, and let $\mathcal{F}_{\boldsymbol{\alpha}}$ be the $\sigma$-algebra on $\Sigma^{\text {gen }}$ generated by $\boldsymbol{\alpha}$. These can be described by $\mathcal{F}_{\alpha}=\left\{\alpha^{-1}(B): B \subset \mathbb{R}\right.$ is Borel $\}$ and similarly for $\mathcal{F}_{\boldsymbol{\alpha}}$. Let $B \subset \mathbb{R}$ be a Borel set and consider the two sets, $\alpha^{-1}(B) \subset \mathcal{G}$ and $\boldsymbol{\alpha}^{-1}(B) \subset \Sigma^{\text {gen }}$, so that $\alpha^{-1}(B)=\left\{n \in \mathcal{G}: \varphi_{l}(n) \in \boldsymbol{\alpha}^{-1}(B)\right\}$. By the assumption, $\boldsymbol{\alpha}^{-1}(B)$ is Jordan since it is an element in $\mathcal{F}_{\boldsymbol{\alpha}}$. Hence, by Corollary 4.11 . 


$$
\mu_{\vec{l}}\left(\boldsymbol{\alpha}^{-1}(B)\right)=d_{\mathcal{G}}\left(\alpha^{-1}(B)\right) .
$$

This proves (5.25) and that every element in $\mathcal{F}_{\alpha}$ has density. In order to conclude that $d_{\mathcal{G}}$ is a probability measure on $\left(\mathcal{G}, \mathcal{F}_{\alpha}\right)$ we are left with showing that $d_{\mathcal{G}}$ is $\sigma$-additive on $\mathcal{F}_{\alpha}$ and $d_{\mathcal{G}}(\mathcal{G})=1$. Since the image of $\alpha$ is countable, then $d_{\mathcal{G}}$ is $\sigma$-additive on $\mathcal{F}_{\alpha}$ if for any subset $J \subset$ Image $(\alpha)$,

$$
d_{\mathcal{G}}\left(\cup_{j \in J} \alpha^{-1}(j)\right)=\sum_{j \in J} d_{\mathcal{G}}\left(\alpha^{-1}(j)\right) .
$$

This follows from 5.26$)$, using $\alpha^{-1}(J)=\cup_{j \in J} \alpha^{-1}(j)$ and the fact that $\mu_{\vec{l}}$ is a measure (hence $\sigma$-additive):

$$
d_{\mathcal{G}}\left(\alpha^{-1}(J)\right)=\mu_{\vec{l}}\left(\boldsymbol{\alpha}^{-1}(J)\right)=\sum_{j \in J} \mu_{\vec{l}}\left(\boldsymbol{\alpha}^{-1}(j)\right)=\sum_{j \in J} d_{\mathcal{G}}\left(\alpha^{-1}(j)\right) .
$$

Applying $(5.26)$ to $B=\mathbb{R}$ gives:

$$
d_{\mathcal{G}}(\mathcal{G})=d_{\mathcal{G}}\left(\alpha^{-1}(\mathbb{R})\right)=\mu_{\vec{l}}\left(\boldsymbol{\alpha}^{-1}(\mathbb{R})\right)=\mu_{\vec{l}}\left(\Sigma^{\text {gen }}\right)=1 .
$$

Therefore, $d_{\mathcal{G}}$ is a probability measure on $\left(\mathcal{G}, \mathcal{F}_{\alpha}\right)$, and so $\alpha$ is a random variable with respect to $d_{\mathcal{G}}$ (see Definition 2.1).

The Lemma above is used in order to show that all the observables discussed in the paper, with the exception of $\rho^{(v)}$, are random variables (see proofs for Theorem 2.2 and Proposition 2.11). In the other direction, the next lemma aids in showing that there are observables (we believe that $\rho^{(v)}$ is such) which cannot be considered as random variables.

Lemma 5.4. Let $\Gamma$ be a standard graph with rationally independent edge lengths $\vec{l}$. Let $\boldsymbol{\alpha}: \Sigma^{\mathrm{gen}} \rightarrow \mathbb{R}$ be a Riemann integrable function (i.e., having discontinuity set of measure zero), and define $\alpha: \mathcal{G} \rightarrow \mathbb{R}$ by

$$
\forall n \in \mathcal{G}, \quad \alpha(n):=\boldsymbol{\alpha}\left(\varphi_{\vec{l}}(n)\right) .
$$

If $\alpha$ is a random variable with respect to $d_{\mathcal{G}}$ then there exists $X \subset \Sigma^{\text {gen }}$ of full measure, $\mu_{\vec{l}}(X)=1$, such that Image $\left(\left.\boldsymbol{\alpha}\right|_{X}\right)$ is countable.

Remark 5.5. As an immediate corollary from the Lemma above we deduce that if $\boldsymbol{\alpha}$ : $\Sigma^{\text {gen }} \rightarrow \mathbb{R}$ is continuous and non-constant on some connected open set, then $\alpha$ is not a random variable. Due to this we cannot generally regard the wavelength capacity, $\rho^{(v)}$, as a random variable. For the wavelength capacity to be considered as a random variable, we need that the corresponding function on the secular manifold, $\boldsymbol{\rho}^{(\boldsymbol{v})}$ be constant on each connected component of $\Sigma^{\text {gen }}$. This is unlikely by (5.23) (see also numerical findings in Section 8) and we even make a stronger conjecture that there is no open set of $\Sigma^{\text {gen }}$ at which $\boldsymbol{\rho}^{(\boldsymbol{v})}$ is constant (see Remark 7.2).

Proof of Lemma 5.4. Observe that the image of $\alpha$ is countable and that for any value $j \in$ Image $(\alpha)$, the set $\boldsymbol{\alpha}^{-1}(j) \subset \Sigma^{\text {reg }}$ is measurable since $\boldsymbol{\alpha}$ is a measurable function (it is even Riemann integrable). Define $X:=\sqcup_{j \in \operatorname{Image}(\alpha)} \boldsymbol{\alpha}^{-1}(j)$. We will show that for all $j \in \operatorname{Image}(\alpha), \mu_{\vec{l}}\left(\boldsymbol{\alpha}^{-1}(j)\right) \geq d_{\mathcal{G}}\left(\alpha^{-1}(j)\right)$ and conclude

$$
\begin{aligned}
\mu_{\vec{l}}(X)=\sum_{j \in \operatorname{Image}(\alpha)} \mu_{\vec{l}}\left(\boldsymbol{\alpha}^{-1}(j)\right) & \geq \sum_{j \in \operatorname{Image}(\alpha)} d_{\mathcal{G}}\left(\alpha^{-1}(j)\right) \\
& =d_{\mathcal{G}}\left(\sqcup_{j \in \operatorname{Image}(\alpha)} \alpha^{-1}(j)\right)=d_{\mathcal{G}}(\mathcal{G})=1,
\end{aligned}
$$


where the first equality follows since $\mu_{\vec{l}}$ is a measure and $X=\sqcup_{j \in \operatorname{Image}(\alpha)} \boldsymbol{\alpha}^{-1}(j)$ is a disjoint countable union; and all equalities on the second lines are by assumption of the lemma that $d_{\mathcal{G}}$ is a probability measure. The statement of the lemma now follows from (5.31) and $\mu_{\vec{l}}(X) \leq \mu_{\vec{l}}\left(\Sigma^{\text {gen }}\right)=1$. It is left to finish the proof by showing that for all $j \in$ Image $(\alpha)$, $\mu_{\vec{l}}\left(\boldsymbol{\alpha}^{-1}(j)\right) \geq d_{\mathcal{G}}\left(\alpha^{-1}(j)\right)$, which we do next.

Let $j \in \operatorname{Image}(\alpha)$ and denote the closure of $\boldsymbol{\alpha}^{-1}(j)$ by $\mathcal{A}=\overline{\boldsymbol{\alpha}^{-1}(j)}$. Notice that the points in $\mathcal{A} \backslash \boldsymbol{\alpha}^{-1}(j)$ are discontinuity points of $\boldsymbol{\alpha}$. Since $\boldsymbol{\alpha}$ was assumed to be Riemann integrable then $\boldsymbol{A} \backslash \boldsymbol{\alpha}^{-1}(j)$ is of measure zero and so

$$
\mu_{\vec{l}}\left(\boldsymbol{\alpha}^{-1}(j)\right)=\mu_{\vec{l}}(\boldsymbol{\mathcal { A }}) .
$$

Using that $\mu_{\vec{l}}$ is a regular measure (Theorem 4.8) we have that for every $\varepsilon>0$, there exist an open set $U_{\varepsilon}$ such that $\mathcal{A} \subset U_{\varepsilon}$ and $\mu_{\vec{l}}\left(U_{\varepsilon} \backslash \mathcal{A}\right)<\varepsilon$. By Urysohn's Lemma there exist a continuous function $f_{\varepsilon}: \Sigma^{\text {gen }} \rightarrow[0,1]$ supported inside $U_{\varepsilon}$ and such that $\left.f_{\varepsilon}\right|_{\mathcal{A}} \equiv 1$. By this construction,

$$
\forall n \in \alpha^{-1}(j), \quad f_{\varepsilon}\left(\varphi_{\vec{l}}(n)\right)=1 .
$$

Denoting $\mathcal{G}(N):=\{n \in \mathcal{G}: n \leq N\}$, we get

$$
\begin{aligned}
d_{\mathcal{G}}\left(\alpha^{-1}(j)\right) & =\lim _{N \rightarrow \infty} \frac{\left|\left\{n \in \mathcal{G}(N): n \in \alpha^{-1}(j)\right\}\right|}{|\mathcal{G}(N)|} \\
& \leq \lim _{N \rightarrow \infty} \frac{1}{|\mathcal{G}(N)|} \sum_{n \in \mathcal{G}(N)} f_{\varepsilon}\left(\varphi_{\vec{l}}(n)\right) \\
& =\int_{\Sigma \text { gen }} f_{\varepsilon} \mathrm{d} \mu_{\vec{l}} \\
& =\int_{\mathcal{A}} f_{\varepsilon} \mathrm{d} \mu_{\vec{l}}+\int_{U_{\varepsilon} \backslash \mathcal{A}} f_{\varepsilon} \mathrm{d} \mu_{\vec{l}} \\
& <\mu_{\vec{l}}(\mathcal{A})+\varepsilon=\mu_{\vec{l}}\left(\boldsymbol{\alpha}^{-1}(j)\right)+\varepsilon,
\end{aligned}
$$

where moving to the third line we use that $\left\{\varphi_{\vec{l}}(n)\right\}_{n \in \mathcal{G}}$ is an equidistributed sequence and $f_{\varepsilon}$ is continuous, and the last equality is due to (5.32). As 5.34 holds for every $\varepsilon>0$, we get the required inequality $\mu_{\vec{l}}\left(\boldsymbol{\alpha}^{-1}(j)\right) \geq d_{\mathcal{G}}\left(\alpha^{-1}(j)\right)$, which finishes the proof.

Remark. We note that the results of the current subsection (Lemmata 5.3 and 5.4) may be similarly proved for a general compact metric space, a measure on it and a corresponding equidistributed sequence. Also, the random variables discussed above are scalars, but the same statements hold for random vector variables whose range of definition is $\mathbb{R}^{n}$ (for any $n \in \mathbb{N})$.

\section{Probability distributions of the Neumann count and the spectral POSITION \\ (proofs of Theorem 2.2, 2, Theorem 2.6 and Proposition 2.11).}

\subsection{Proving the existence and symmetry of the probability distributions of $\omega$ and $N^{(v)}$}

(Theorem 2.2, (2) and Proposition 2.11).

Proof of Theorem 2.2, (2). We wish to apply Lemma 5.3 in order to show that the Neumann surplus, $\omega: \mathcal{G} \rightarrow \mathbb{Z}$, is a random variable with respect to $d_{\mathcal{G}}$. For this purpose, we use the function $\boldsymbol{\omega}: \Sigma^{\text {gen }} \rightarrow \mathbb{Z}$, whose existence and properties were established in Lemma 
5.2 and in particular, $\omega(n)=\boldsymbol{\omega}\left(\varphi_{\vec{l}}(n)\right)$ for all $n \in \mathcal{G}$. By Lemma 5.2 , the function $\boldsymbol{\omega}$ is constant on connected components of $\Sigma^{\text {gen }}$. Let $\mathcal{F}_{\boldsymbol{\omega}}$ be the $\sigma$-algebra generated by $\boldsymbol{\omega}$. Then each element in $\mathcal{F}_{\boldsymbol{\omega}}$ is a union of connected components of $\Sigma^{\text {gen }}$. $\Sigma^{\text {gen }}$ has a finite number of connected components, by Lemma 4.3 , and each of them is both open and close since $\Sigma^{\text {gen }}$ is locally connected. Hence, each element in $\mathcal{F}_{\boldsymbol{\omega}}$ is also open and closed and as such has no boundary and is Jordan. This is exactly the condition in Lemma 5.3, by which we get that $\omega$ is a random variable with respect to $d_{\mathcal{G}}$. Furthermore, $\omega$ is a finite random variable. Indeed, by the above $\operatorname{Image}(\boldsymbol{\omega})$ is finite and so Image $(\omega)$ is finite as well ${ }^{7}$. This completes the proof of part 2a of the theorem.

To prove the next two parts of the theorem note that another implication of Lemma 5.3 is

$$
\forall j \in \operatorname{Image}(\boldsymbol{\omega}), \quad d_{\mathcal{G}}\left(\omega^{-1}(j)\right)=\mu_{\vec{l}}\left(\boldsymbol{\omega}^{-1}(j)\right) .
$$

Let $j$ be such that $\omega^{-1}(j) \neq \emptyset$, so $\boldsymbol{\omega}^{-1}(j) \neq \emptyset$. As argued above, $\boldsymbol{\omega}^{-1}(j)$ is an open set. This together with $\mu_{\vec{\imath}}$ being strictly positive (Theorem 4.8, (2)) yields $\mu_{\vec{l}}\left(\boldsymbol{\omega}^{-1}(j)\right)>0$. By (6.1), this proves part (2b) of the theorem.

Finally, to prove part (2c) of the theorem, we use the inversion map, $\mathcal{I}: \vec{\kappa} \mapsto[-\vec{\kappa}]$ which acts on $\Sigma^{\text {gen }}$. Since by Lemma 4.12, (2), $\mathcal{I}$ preserves the measure $\mu_{\vec{l}}$ we have

$$
\begin{aligned}
\forall j \quad \mu_{\vec{l}}\left(\boldsymbol{\omega}^{-1}(j)\right) & =\mu_{\vec{l}}\left(\mathcal{I}\left(\boldsymbol{\omega}^{-1}(j)\right)\right) \\
& =\mu_{\vec{l}}\left((\boldsymbol{\omega} \circ \mathcal{I})^{-1}(j)\right) \\
& =\mu_{\vec{l}}\left(\boldsymbol{\omega}^{-1}(\beta-|\partial \Gamma|-j)\right),
\end{aligned}
$$

where in the second line we used that $\mathcal{I}$ is an involution and the third line is obtained from (5.6), which reads $(\boldsymbol{\omega} \circ \mathcal{I})(\vec{\kappa})=\beta-|\partial \Gamma|-\boldsymbol{\omega}(\vec{\kappa})$. Combining (6.2) with (6.1) gives the desired symmetry of the probability distribution.

The next proof is quite similar to the previous one. The only essential difference ${ }^{8}$ is in introducing an auxiliary random variable with respect to $d_{\mathcal{G}}$.

Proof of Proposition 2.11. We start by recalling the function $N^{(v)}: \Sigma^{\text {gen }} \rightarrow \mathbb{N}$, whose existence and properties were established in Lemma 5.2 and introduce $\widetilde{N}^{(v)}: \mathcal{G} \rightarrow \mathbb{N}$ by

$$
\forall n \in \mathcal{G}, \quad \widetilde{N}^{(v)}(n)=\boldsymbol{N}^{(v)}\left(\varphi_{\vec{l}}(n)\right) .
$$

Following exactly the same arguments as in the beginning of the preceding proof (proof of Theorem 2.2. (2) ), we get that $\widetilde{N}^{(v)}$ is a finite random variable with respect to $d_{\mathcal{G}}$. We need to obtain a similar statement for $N^{(v)}$. To do so, we note that by 5.4 and 6.3

$$
\forall n \in \mathcal{G} \text { s.t. } n \geq 2 \frac{|\Gamma|}{L_{\min }}, \quad N^{(v)}(n)=\widetilde{N}^{(v)}(n),
$$

where $L_{\min }$ is the minimal edge length of $\Gamma$ and $|\Gamma|$ is the sum of all edge lengths. Thanks to 6.4, for any Borel set $B \subset \mathbb{R}$ the pre-images $\left(N^{(v)}\right)^{-1}(B)$ and $\left(\widetilde{N}^{(v)}\right)^{-1}(B)$ differ by a finite number of elements from $\mathcal{G}_{\text {singletons. }}$. Since the density of $\left(\widetilde{N}^{(v)}\right)^{-1}(B)$ exists (because $\widetilde{N}^{(v)}$ is a random variable with respect to $d_{\mathcal{G}}$ ), so does the density of $\left(N^{(v)}\right)^{-1}(B)$, and both are equal. A similar argument shows that $d_{\mathcal{G}}$ is a probability measure on the

\footnotetext{
${ }^{7}$ This was also proven in Theorem 2.2, (1).

${ }^{8}$ This difference is due to the special role which is played by a finite number of eigenvalues appearing in the beginning of the spectrum - see details within the proof.
} 
$\sigma$-algebra generated by $N^{(v)}$, and so $N^{(v)}$ is a random variable with respect to $d_{\mathcal{G}}$. It is finite since $\widetilde{N}^{(v)}$ is finite and their images may differ by at most a finite number of values. This proves the first part of the proposition.

To prove the second part, we use the inversion map, $\mathcal{I}: \vec{\kappa} \mapsto[-\vec{\kappa}]$ which acts on $\Sigma^{\text {gen }}$. By Lemma $2 \mathcal{I}$ preserves the measure $\mu_{\vec{l}}$, and so

$$
\begin{aligned}
\forall j \quad \mu_{\vec{l}}\left(\left(\boldsymbol{N}^{(\boldsymbol{v})}\right)^{-1}(j)\right) & =\mu_{\vec{l}}\left(\mathcal{I}\left(\left(\boldsymbol{N}^{(\boldsymbol{v})}\right)^{-1}(j)\right)\right) \\
& =\mu_{\vec{l}}\left(\left(\boldsymbol{N}^{(\boldsymbol{v})} \circ \mathcal{I}\right)^{-1}(j)\right) \\
& =\mu_{\vec{l}}\left(\left(\boldsymbol{N}^{(\boldsymbol{v})}\right)^{-1}(\operatorname{deg}(v)-j)\right),
\end{aligned}
$$

where in the second line we used that $\mathcal{I}$ is an involution and the third line is obtained from $(5.7)$, which reads $\left(\boldsymbol{N}^{(\boldsymbol{v})} \circ \mathcal{I}\right)(\vec{\kappa})=\operatorname{deg}(v)-\boldsymbol{N}^{(\boldsymbol{v})}(\vec{\kappa})$. To finish the proof observe that

$$
\forall j \quad d_{\mathcal{G}}\left(\left(N^{(v)}\right)^{-1}(j)\right)=d_{\mathcal{G}}\left(\left(\tilde{N}^{(v)}\right)^{-1}(j)\right)=\mu_{\vec{l}}\left(\left(\boldsymbol{N}^{(v)}\right)^{-1}(j)\right),
$$

where the first equality was shown above, and the second follows from 5.25 in Lemma 5.3 .

\subsection{The Neumann count distribution of $(3,1)$-regular trees (proof of Theorem 2.6).}

Theorem 2.6 is proved using the following.

Definition 6.1. A bridge (or cut-edge) of a graph $\Gamma$ is an edge of $\Gamma$, whose removal increases the number of connected components of $\Gamma$.

Proposition 6.2. Let $\Gamma$ be a standard graph with rationally independent edge lengths. Let $\widetilde{\Gamma}$ be a sub-graph of $\Gamma$ such that all edges between $\widetilde{\Gamma}$ and $\Gamma \backslash \widetilde{\Gamma}$ are bridges of $\Gamma$. Let $\tilde{v}$ be a vertex of $\widetilde{\Gamma}$ with degree $\operatorname{deg}(\tilde{v})>1$ and denote its spectral position random variable by $N^{(\tilde{v})}$. Further denote by $\vec{N}^{(\Gamma \backslash \widetilde{\Gamma})}$ the vector of all the spectral position random variables of interior vertices (of $\Gamma$ ) in $\Gamma \backslash \widetilde{\Gamma}$. Then

$$
\forall 1 \leq j \leq d_{\tilde{v}}-1, \quad \mathbb{P}\left(N^{(\tilde{v})}=j \mid \vec{N}^{(\Gamma \backslash \widetilde{\Gamma})}\right)=\mathbb{P}\left(N^{(\tilde{v})}=d_{\tilde{v}}-j \mid \vec{N}^{(\Gamma \backslash \widetilde{\Gamma})}\right) .
$$

Namely, $N^{(\tilde{v})}$ is symmetrically distributed, independently of all spectral position random variables of $\Gamma \backslash \widetilde{\Gamma}$.

Before proving the proposition above we first point out and prove a few of its implications, the last of which is exactly the statement of Theorem 2.6.

Corollary 6.3. Let $\Gamma$ be a standard graph with rationally independent edge lengths.

(1) If $\Gamma$ is a tree graph and $v, u \in \mathcal{V} \backslash \partial \Gamma$ are two different vertices then their spectral positions are uncorrelated random variables.

(2) If $\Gamma$ is a $(3,1)$-regular tree graph then its spectral position random variables, $\left\{N^{(v)}\right\}_{v \in \mathcal{V} \backslash \partial \Gamma}$ are mutually independent.

(3) If $\Gamma$ is a $(3,1)$-regular tree graph then the probability distribution of the random variable $-\omega-1$ is binomial, $\operatorname{Bin}\left(|\partial \Gamma|-2, \frac{1}{2}\right)$. 
Proof. Proof of part (11). To apply Proposition 6.2 choose for $\widetilde{\Gamma}$ any sub-graph of $\Gamma$ which contains $v$, but does not contain $u$ (we may even choose $\widetilde{\Gamma}$ to be just $v$ ). Since $\Gamma$ is a tree, each of its edges is a bridge and hence indeed any such choice of $\widetilde{\Gamma}$ satisfies the conditions of Proposition 6.2.

Now, as a particular case of 6.6 we get that

$$
\forall 1 \leq j \leq d_{v}-1, \quad \mathbb{P}\left(N^{(v)}=j \mid N^{(u)}\right)=\mathbb{P}\left(N^{(v)}=d_{v}-j \mid N^{(u)}\right)
$$

and so

$$
\begin{gathered}
\mathbb{E}\left[\left(N^{(v)}-\frac{d_{v}}{2}\right)\left(N^{(u)}-\frac{d_{u}}{2}\right)\right]=\sum_{j=1}^{d_{v}-1} \sum_{i=1}^{d_{u}-1}\left(j-\frac{d_{v}}{2}\right)\left(i-\frac{d_{u}}{2}\right) \mathbb{P}\left(N^{(v)}=j \wedge N^{(u)}=i\right) \\
=\sum_{j=1}^{d_{v}-1} \sum_{i=1}^{d_{u}-1}\left(j-\frac{d_{v}}{2}\right)\left(i-\frac{d_{u}}{2}\right) \mathbb{P}\left(N^{(v)}=j \mid N^{(u)}=i\right) \mathbb{P}\left(N^{(u)}=i\right) \\
=\sum_{i=1}^{d_{u}-1}\left(i-\frac{d_{u}}{2}\right) \mathbb{P}\left(N^{(u)}=i\right) \sum_{j=1}^{d_{v}-1}\left(j-\frac{d_{v}}{2}\right) \mathbb{P}\left(N^{(v)}=j \mid N^{(u)}=i\right)=0,
\end{gathered}
$$

where the sum over $j$ in the last line contains terms which cancel each other by (6.7) and so the whole sum vanishes. By Proposition 2.11, (2) we have $\mathbb{E}\left[N^{(v)}-\frac{d_{v}}{2}\right]=\mathbb{E}\left[N^{(u)}-\frac{d_{u}}{2}\right]=0$, and so $\mathbb{E}\left[\left(N^{(v)}-\frac{d_{v}}{2}\right)\left(N^{(u)}-\frac{d_{u}}{2}\right)\right]=\mathbb{E}\left[N^{(v)}-\frac{d_{v}}{2}\right] \mathbb{E}\left[N^{(u)}-\frac{d_{u}}{2}\right]$ and $N^{(v)}-\frac{d_{v}}{2}, N^{(u)}-\frac{d_{u}}{2}$ are uncorrelated random variables. We conclude that $N^{(v)}, N^{(u)}$ are uncorrelated random variables.

We remark that the calculation in (6.8) may be extended to include not just two individual vertices, but any two sets of vertices, and thus prove a more general statement.

Proof of part (2)

Let $\tilde{v}$ be a vertex of $\Gamma \backslash \partial \Gamma$. Namely, $\tilde{v}$ is not a boundary vertex, and so as $\Gamma$ is $(3,1)$ regular, $d_{\tilde{v}}=3$. Choose $\widetilde{\Gamma}$ to be $\tilde{v}$ and apply 6.6 in Proposition 6.2 , with $d_{\tilde{v}}=3$ and $j=1$ to get

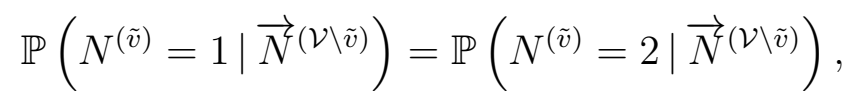

where $\vec{N}(\mathcal{V} \backslash \tilde{v})$ indicates the vector of random variables of spectral positions of all nonboundary vertices of $\Gamma$ except $\tilde{v}$. Now, as $N^{(\tilde{v})}$ gets only the two values 1 and 2, we obtain that it is independent from all other random variables in $\vec{N}^{(\mathcal{V} \backslash \tilde{v})}$, as stated in part (2) of the Proposition.

Proof of part (3)

Applying (2.12) in Proposition 2.13 gives

$$
\sum_{v \in \mathcal{V} \backslash \partial \Gamma} N^{(v)}=\sigma-\omega+(|\mathcal{E}|-|\partial \Gamma|) .
$$

As $\Gamma$ is a tree graph, we have $|\mathcal{E}|=|\mathcal{V}|-1$ and $\sigma \equiv 0$ (see $(3.2)$ ) and so

$$
\omega=-\sum_{v \in \mathcal{V} \backslash \partial \Gamma} N^{(v)}+|\mathcal{V} \backslash \partial \Gamma|-1,
$$


which we rewrite as

$$
-\omega-1=\sum_{v \in \mathcal{V} \backslash \partial \Gamma}\left(N^{(v)}-1\right) .
$$

The right hand side of 6.12 is a sum of independent random variables, $N^{(v)}-1$, as is proven in the previous part. In addition, by Proposition 2.11, (2), each $N^{(v)}-1$ is a symmetric Bernoulli random variable (it obtains 0 and 1, each with probability $\frac{1}{2}$ ), and so their sum is distributed as $\operatorname{Bin}\left(|\mathcal{V} \backslash \partial \Gamma|, \frac{1}{2}\right)$. To prove the required statement we only need to show that $|\mathcal{V} \backslash \partial \Gamma|=|\partial \Gamma|-2$, which follows from

$$
|\mathcal{E}|=|\mathcal{V}|-1 \quad \text { and } \quad 2|\mathcal{E}|=3|\mathcal{V} \backslash \partial \Gamma|+|\partial \Gamma|
$$

The first equality above comes from $\Gamma$ being a tree and the second from summing all vertex degrees and using that $\Gamma$ is $(3,1)$-regular.

We proceed to prove Proposition 6.2. The next lemma forms an essential tool towards this proof. This lemma is of similar spirit to Lemma 4.12, it provides an involution of the secular manifold, which is applicable for a graph that contains a bridge.

Lemma 6.4. [5, Lem. 4.15]

Let $\Gamma$ be a standard connected graph. Let e be a bridge of $\Gamma$ and denote by $\Gamma_{1}, \Gamma_{2}$ the sub-graphs of $\Gamma$ which are the two connected components of $\Gamma \backslash\{e\}$. Let $v$ be the vertex of $\Gamma_{1}$ which is connected to e. There exists a map $\mathcal{R}_{v, e}: \mathbb{T}^{E} \rightarrow \mathbb{T}^{E}$ (which we call the bridge-inversion map) such that

(1) The bridge-inversion is an involution, $\left(\mathcal{R}_{v, e}\right)^{2}=\mathrm{Id}$.

(2) Each of the manifolds, $\Sigma, \Sigma^{\mathrm{reg}}$ and $\Sigma^{\mathrm{gen}}$ is invariant under the bridge-inversion.

(3) The restriction of the bridge-inversion to the generic part of the secular manifold, $\left.\mathcal{R}_{v, e}\right|_{\Sigma_{\text {gen }}}$, is a map which preserves the Barra-Gaspard measure, $\mu_{\vec{l}}$.

(4) For every $u \in \mathcal{V}$ and $e \in \mathcal{E}_{u}$ the functions $p_{u, u}$ and $q_{u, u, e}$ (defined in Lemmas 4.2 and 4.12) satisfy the following symmetry $\backslash$ anti-symmetry with respect to $\mathcal{R}_{v, e}$ :

$$
\forall \vec{\kappa} \in \Sigma^{\mathrm{reg}}, \quad p_{u, u}\left(\mathcal{R}_{v, e}(\vec{\kappa})\right)=p_{u, u}(\vec{\kappa}),
$$

and

$$
\forall \vec{\kappa} \in \Sigma^{\mathrm{reg}}, \quad q_{u, u, e}\left(\mathcal{R}_{v, e}(\vec{\kappa})\right)=\left\{\begin{array}{ll}
q_{u, u, e}(\vec{\kappa}) & u \in \mathcal{V}_{1} \\
-q_{u, u, e}(\vec{\kappa}) & u \in \mathcal{V}_{2}
\end{array} .\right.
$$

The proof of this lemma may be found within the proofs of [5, Lem. 4.15] and [3, Lem. $4.42]$.

Proof of Proposition 6.2. This proof is somewhat similar in spirit to the one of Proposition 2.11, (2), where it was proven that the spectral position of any vertex is a symmetric random variable. Yet, to prove that it is not only symmetric, but independently symmetric (when conditioning on spectral position of other vertices), requires some more work.

Let $\Gamma, \widetilde{\Gamma}$ and $\tilde{v}$ as in the statement of the proposition. Let $j \in \mathbb{N}$ and let $\vec{n}$ be a vector of natural numbers of length $|\Gamma \backslash(\partial \Gamma \cup \widetilde{\Gamma})|$. So, to prove the proposition we need to show that

$$
\mathbb{P}\left(N^{(\tilde{v})}=j \wedge \vec{N}^{(\Gamma \backslash \widetilde{\Gamma})}=\vec{n}\right)=\mathbb{P}\left(N^{(\tilde{v})}=d_{\tilde{v}}-j \wedge \vec{N}^{(\Gamma \backslash \widetilde{\Gamma})}=\vec{n}\right)
$$


Translating (6.16) into densities gives

$$
d_{\mathcal{G}}\left(\left(N^{(\tilde{v})}\right)^{-1}(j) \cap\left(\vec{N}^{(\Gamma \backslash \widetilde{\Gamma})}\right)^{-1}(\vec{n})\right)=d_{\mathcal{G}}\left(\left(N^{(\tilde{v})}\right)^{-1}\left(d_{\tilde{v}}-j\right) \cap\left(\vec{N}^{(\Gamma \backslash \widetilde{\Gamma})}\right)^{-1}(\vec{n})\right) .
$$

This may be further translated into the corresponding Barra-Gaspard measure on the secular manifold,

$$
\mu_{\vec{l}}\left(\left(\boldsymbol{N}^{(\tilde{\boldsymbol{v}})}\right)^{-1}(j) \cap\left(\overrightarrow{\boldsymbol{N}}^{(\boldsymbol{\Gamma} \backslash \widetilde{\boldsymbol{\Gamma}})}\right)^{-1}(\vec{n})\right)=\mu_{\vec{l}}\left(\left(\boldsymbol{N}^{(\tilde{\boldsymbol{v}})}\right)^{-1}\left(d_{\tilde{v}}-j\right) \cap\left(\overrightarrow{\boldsymbol{N}}^{(\boldsymbol{\Gamma} \backslash \widetilde{\boldsymbol{\Gamma}})}\right)^{-1}(\vec{n})\right)
$$

where $\boldsymbol{N}^{(\tilde{\boldsymbol{v}})}$ and $\overrightarrow{\boldsymbol{N}}^{(\Gamma \backslash \widetilde{\boldsymbol{\Gamma}})}$ are the spectral position functions on the secular manifold which were introduced in Lemma 5.2. The argument for translating the natural densities, $d_{\mathcal{G}}$ to the Barra-Gaspard measure, $\mu_{\vec{l}}$, is as in the proof of Proposition 2.11 (see (6.5) there).

We proceed to prove 6.18). Let $\left\{e_{i}\right\}_{i=1}^{m}$ be all the edges which connect $\widetilde{\Gamma}$ to $\Gamma \backslash \widetilde{\Gamma}$. By assumption, all those edges are bridges. For each $e_{i}$ denote by $v_{i}$ the vertex of $\widetilde{\Gamma}$ which is connected to $e_{i}$. Further denote by $\Gamma_{i}$ the connected component of $\Gamma \backslash e_{i}$ which does not contain $\widetilde{\Gamma}$. With those notations, we may write the following decomposition of the graph

$$
\Gamma=\widetilde{\Gamma} \cup \bigcup_{i=1}^{m} e_{i} \cup \bigcup_{i=1}^{m} \Gamma_{i},
$$

which is disjoint up to the vertices at the endpoints of the bridges $\left\{e_{i}\right\}_{i=1}^{m}$. Consider the following composition of involutions, $\mathcal{I} \cdot \mathcal{R}_{v_{1}, e_{1}} \ldots \cdot \mathcal{R}_{v_{m}, e_{m}}$ and note that all those involutions are $\mu_{\vec{l}}$ measure preserving (Lemma 4.12, (2) and Lemma 6.4.(3)). Therefore, in order to prove (6.18) it is enough to show that

$$
\mathcal{I} \circ \mathcal{R}_{v_{1}, e_{1}} \circ \ldots \circ \mathcal{R}_{v_{m}, e_{m}}\left(\left(\boldsymbol{N}^{(\tilde{\boldsymbol{v}})}\right)^{-1}(j)\right)=\left(\boldsymbol{N}^{(\tilde{\boldsymbol{v}})}\right)^{-1}\left(d_{\tilde{\boldsymbol{v}}}-j\right)
$$

and

$$
\mathcal{I} \circ \mathcal{R}_{v_{1}, e_{1}} \circ \ldots \circ \mathcal{R}_{v_{m}, e_{m}}\left(\left(\overrightarrow{\boldsymbol{N}}^{(\Gamma \backslash \widetilde{\Gamma})}\right)^{-1}(\vec{n})\right)=\left(\overrightarrow{\boldsymbol{N}}^{(\Gamma \backslash \widetilde{\Gamma})}\right)^{-1}(\vec{n}) .
$$

To verify both, we recall that (see $(5.20)$

$$
\forall v \in \mathcal{V}, \quad \boldsymbol{N}^{(v)}(\vec{\kappa})=\frac{1}{2} d_{v}-\frac{1}{2} \sum_{e \in \mathcal{E}_{v}} \operatorname{sgn}\left(q_{v, v, e}(\vec{\kappa})\right)
$$

and that (by Lemmas 4.12 and 6.4)

$$
\begin{gathered}
\forall v \in \mathcal{V} \forall e \in \mathcal{E}_{v}, \quad q_{v, v, e}(\mathcal{I}(\vec{\kappa}))=-q_{v, v, e}(\vec{\kappa}), \\
\forall v \in \mathcal{V} \forall e \in \mathcal{E}_{v}, \quad q_{v, v, e}\left(\mathcal{R}_{v_{i}, e_{i}}(\vec{\kappa})\right)=\left\{\begin{array}{ll}
q_{v, v, e}(\vec{\kappa}) & v \in \Gamma \backslash \Gamma_{i} \\
-q_{v, v, e}(\vec{\kappa}) & v \in \Gamma_{i}
\end{array} .\right.
\end{gathered}
$$

Each vertex $v \in \Gamma \backslash \widetilde{\Gamma}$ belongs to exactly one of the $\Gamma_{i}$ graphs, so by 6.23) and 6.24) we have that 6.22 is invariant under $\mathcal{I} \circ \mathcal{R}_{v_{1}, e_{1}} \circ \ldots \circ \mathcal{R}_{v_{m}, e_{m}}$ (as each $\operatorname{sgn}\left(q_{v, v, e}\right)$ is inverted twice by its action and hence unchanged). This implies

$$
\boldsymbol{N}^{(\boldsymbol{v})}\left(\mathcal{I} \circ \mathcal{R}_{v_{1}, e_{1}} \circ \ldots \circ \mathcal{R}_{v_{m}, e_{m}}(\vec{\kappa})\right)=\boldsymbol{N}^{(\boldsymbol{v})}(\vec{\kappa}),
$$

which proves 6.21 . Similarly, any vertex $\tilde{v} \in \widetilde{\Gamma}$ is not contained in any of the $\Gamma_{i}$ graphs and so by 6.23$)$ and $(6.24)$ we have that each $\operatorname{sgn}\left(q_{\tilde{v}, \tilde{v}, e}\right)$ in 6.22 is inverted once by the 
action of $\mathcal{I} \circ \mathcal{R}_{v_{1}, e_{1}} \circ \ldots \circ \mathcal{R}_{v_{m}, e_{m}}$. This implies

$$
\boldsymbol{N}^{(\tilde{v})}\left(\mathcal{I} \circ \mathcal{R}_{v_{1}, e_{1}} \circ \ldots \circ \mathcal{R}_{v_{m}, e_{m}}(\vec{\kappa})\right)=\operatorname{deg}(v)-\boldsymbol{N}^{(\tilde{v})}(\vec{\kappa})
$$

and proves 6.20.

\section{Probability distribution of the WaVelength CApacity \\ (Proof of Proposition 2.12)}

The proof of Proposition 2.12 is based on the existence and properties of the function $\rho^{(v)}$ defined on $\Sigma^{\text {gen }}$ (Lemma 5.2). In the proof we need to argue that some level sets of $\rho^{(v)}$ are of measure zero. To show it we use that $\Sigma^{\text {gen }}$ (and actually even $\Sigma^{\text {reg }}$ ) is a real analytic manifold [25, 5, 3] and the following.

Lemma 7.1. Let $\mathcal{M} \subset \mathbb{T}^{E}$ be a connected real analytic manifold of dimension $E-1$ and let $d \mu$ be its volume element. Let $g$ be a real analytic function on $\mathcal{M}$.

(1) Either $\left.g\right|_{\mathcal{M}} \equiv 0$ or the zero set of $g$ is of co-dimension at least one in $\mathcal{M}$.

(2) If $g$ is not constant on $\mathcal{M}$ then the pre-image by $g$ of any set of (Lebesgue) measure zero is of $\mu$-measure zero.

Proof. Denote the zero set of $g$ by $Z_{g}:=\{\vec{k} \in \mathcal{M} \mid g(\vec{\kappa})=0\}$. Choose some atlas $\left\{\left(U_{n}, \varphi_{n}\right)\right\}$ for $\mathcal{M}$, such that for all $n, \varphi_{n}: U_{n} \rightarrow O_{n} \subset \mathbb{R}^{E-1}$. Since $\mathcal{M}$ is a real analytic manifold and $g$ is real analytic, we get that for all $n, g_{n}:=g \circ \varphi_{n}^{-1}$ is real analytic on $O_{n} \subset \mathbb{R}^{E-1}$. By [40, Prop. 3] we get that either $\left.g_{n}\right|_{O_{n}} \equiv 0$ or its zero set, $\varphi_{n}\left(Z_{g} \cap U_{n}\right)$ is of co-dimension at least one in $\mathbb{R}^{E-1}$. Hence, either $\left.g\right|_{U_{n}} \equiv 0$ or $Z_{g} \cap U_{n}$ has a positive co-dimension in $U_{n}$. We use this dichotomy to define

$$
\begin{aligned}
& N_{1}:=\left\{n: U_{n} \subset Z_{g}\right\} \\
& N_{2}:=\left\{n: Z_{g} \cap U_{n} \text { has a positive co-dimension in } U_{n}\right\}
\end{aligned}
$$

and

$$
A=\cup_{n \in N_{1}} U_{n}, \quad B=\cup_{n \in N_{2}} U_{n} .
$$

Clearly, $A \cap B=\emptyset$ and so we get the partition of $\mathcal{M}=A \sqcup B$ as a union of disjoint open sets. Since $\mathcal{M}$ is connected we get either $A=\mathcal{M}$ which implies $\left.g\right|_{\mathcal{M}} \equiv 0$ or $B=\mathcal{M}$ which implies that $Z_{g}$ is of positive co-dimension in $\mathcal{M}$ and proves the first part of the lemma.

To prove the second part of the Lemma, consider the gradient of $g_{n}$ which we denote by $\nabla g_{n}: O_{n} \rightarrow \mathbb{R}^{E-1}$ and its zero set which we denote by $Z_{\nabla g_{n}}$. Each of the components of $\nabla g_{n}$ is real analytic, since $g_{n}$ itself is real analytic. Next, apply the argument from the first part of the proof for each component $\partial_{i} g_{n}$ of the gradient. We get that either the zero set of $\partial_{i} g_{n}$ is $O_{n}$ or it is of positive co-dimension. If for all components $\partial_{i} g_{n}$ the zero set is $O_{n}$ this implies that $g_{n}$ is constant on $U_{n}$. We denote

$$
\begin{aligned}
& N_{3}:=\left\{n: g_{n} \text { is constant }\right\} \\
& N_{4}:=\left\{n: g_{n} \text { is not constant }\right\}
\end{aligned}
$$

and

$$
C=\cup_{n \in N_{3}} U_{n}, \quad D=\cup_{n \in N_{4}} U_{n} .
$$

Exactly as above we get that since $\mathcal{M}$ is connected either $C=\mathcal{M}$ or $D=\mathcal{M}$. In the former case $g$ is constant on $\mathcal{M}$. But, $g$ is not constant by the assumption of our lemma 
and so $D=\mathcal{M}$ and $g_{n}$ is not constant (for any $n$ ). By the argument above, this means that for each $n$ there exists $\partial_{i} g_{n}$, whose zero set is of positive co-dimension, which implies that the zero set of the gradient, $\nabla g_{n}$ is of positive co-dimension and hence is of measure zero (with respect to Lebesgue measure on $\mathbb{R}^{E-1}$ ). This is the condition stated in 44, Thm. 1], from which we deduce that if $B \subset \mathbb{R}$ is of measure zero then $g_{n}^{-1}(B) \subset \mathbb{R}^{E-1}$ is of measure zero. Therefore, $\varphi_{n}^{-1}\left(g_{n}^{-1}(B)\right)=g^{-1}(B) \cap U_{n}$ is of $\mu$-measure zero and this holds for all charts (since $D=\mathcal{M})$. Hence, we get that $g^{-1}(B)$ is of $\mu$-measure zero.

Proof of Proposition 2.12. The first step in the proof is to express the LHS of (2.11) as

$$
\forall a<b, \quad d_{\mathcal{G}}\left(\left(\rho^{(v)}\right)^{-1}((a, b))\right)=\mu_{\vec{l}}\left(\left(\boldsymbol{\rho}^{(v)}\right)^{-1}((a, b))\right),
$$

where $\rho^{(v)}: \Sigma^{\text {gen }} \rightarrow \mathbb{R}$ is the real analytic function whose existence and properties specified in Lemma 5.2. In order to show 7.1) we need to argue that $\left(\boldsymbol{\rho}^{(\boldsymbol{v})}\right)^{-1}((a, b))$ is a Jordan set and then apply Corollary 4.11 .

To prove that $\left(\boldsymbol{\rho}^{(\boldsymbol{v})}\right)^{-1}((a, b))$ is a Jordan set, we start by noting that $\boldsymbol{\rho}^{(\boldsymbol{v})}: \Sigma^{\text {gen }} \rightarrow \mathbb{R}$ is a continuous function (being even real analytic). Hence, for every open $I \subset \mathbb{R}$

$$
\begin{aligned}
\partial\left\{\left(\boldsymbol{\rho}^{(v)}\right)^{-1}(I)\right\} & =\overline{\left(\boldsymbol{\rho}^{(v)}\right)^{-1}(I) \backslash}\left(\boldsymbol{\rho}^{(v)}\right)^{-1}(I) \\
& \subset\left(\boldsymbol{\rho}^{(\boldsymbol{v})}\right)^{-1}(\bar{I}) \backslash\left(\boldsymbol{\rho}^{(\boldsymbol{v})}\right)^{-1}(I)=\left(\boldsymbol{\rho}^{(v)}\right)^{-1}(\partial I)
\end{aligned}
$$

where ${ }^{-}$and $\partial$ denote, correspondingly, the closure and boundary operators (in $\mathbb{R}$ or in $\Sigma^{\text {gen }}$ according to the context). Now using that $I$ is open we get $I \cap \partial I=\emptyset$ and in particular, $\left(\boldsymbol{\rho}^{(\boldsymbol{v})}\right)^{-1}(I) \cap\left(\boldsymbol{\rho}^{(\boldsymbol{v})}\right)^{-1}(\partial I)=\emptyset$. As the first set is open and the second is closed we have

$$
\partial\left\{\left(\boldsymbol{\rho}^{(\boldsymbol{v})}\right)^{-1}(I)\right\} \cap\left\{\left(\boldsymbol{\rho}^{(\boldsymbol{v})}\right)^{-1}(\partial I)\right\}^{\circ}=\emptyset,
$$

where ${ }^{\circ}$ denotes the interior operator. Combining (7.3) with $(7.2)$ yields the stronger inclusion

$$
\partial\left\{\left(\boldsymbol{\rho}^{(\boldsymbol{v})}\right)^{-1}(I)\right\} \subset \partial\left\{\left(\boldsymbol{\rho}^{(\boldsymbol{v})}\right)^{-1}(\partial I)\right\} .
$$

In particular, choosing $I=(a, b)$ gives

$$
\partial\left\{\left(\boldsymbol{\rho}^{(\boldsymbol{v})}\right)^{-1}((a, b))\right\} \subset \partial\left\{\left(\boldsymbol{\rho}^{(\boldsymbol{v})}\right)^{-1}(a)\right\} \cup \partial\left\{\left(\boldsymbol{\rho}^{(\boldsymbol{v})}\right)^{-1}(b)\right\} .
$$

Hence, since $\Sigma^{\text {gen }}$ has finitely many connected components (Lemma 4.3) and by Lemma 7.1. we get that each of $\left\{\left(\boldsymbol{\rho}^{(\boldsymbol{v})}\right)^{-1}(a)\right\}$ and $\left\{\left(\boldsymbol{\rho}^{(\boldsymbol{v})}\right)^{-1}(b)\right\}$ is a finite union of connected components of $\Sigma^{\text {gen }}$ and sets of positive co-dimension in $\Sigma^{\text {gen }}$. The boundary of a connected component of $\Sigma^{\text {gen }}$ is an empty set $^{9}$. The boundary of a set of positive co-dimension has itself positive co-dimension and is therefore of measure zero. Hence, the boundaries $\partial\left\{\left(\boldsymbol{\rho}^{(\boldsymbol{v})}\right)^{-1}(a)\right\}$ and $\partial\left\{\left(\boldsymbol{\rho}^{(\boldsymbol{v})}\right)^{-1}(b)\right\}$ are of measure zero and by 7.5 we conclude that $\partial\left\{\left(\boldsymbol{\rho}^{(\boldsymbol{v})}\right)^{-1}((a, b))\right\}$ is also of measure zero. We conclude that $\left(\boldsymbol{\rho}^{(\boldsymbol{v})}\right)^{-1}((a, b))$ is indeed

\footnotetext{
${ }^{9}$ We have shown in the proof of Theorem 2.2, (2) that a connected component of $\Sigma^{\text {gen }}$ is open and closed and hence it has an empty boundary.
} 
a Jordan set and may now apply Corollary 4.11 and get

$$
d_{\mathcal{G}}\left(\varphi_{\vec{l}}^{-1}\left(\left(\boldsymbol{\rho}^{(\boldsymbol{v})}\right)^{-1}((a, b))\right)\right)=\mu_{\vec{l}}\left(\left(\boldsymbol{\rho}^{(\boldsymbol{v})}\right)^{-1}((a, b))\right) .
$$

From here, we obtain (7.1) from the beginning of the proof by observing that the sets $\varphi_{\vec{l}}^{-1}\left(\left(\boldsymbol{\rho}^{(\boldsymbol{v})}\right)^{-1}((a, b))\right)$ and $\left(\rho^{(v)}\right)^{-1}((a, b))$ differ by at most finitely many elements. Indeed, this was shown in $(5.5)$ of Lemma 5.2 .

Having shown (7.1), the first part of the Proposition is therefore equivalent to

$$
\mu_{\vec{l}}\left(\left(\boldsymbol{\rho}^{(\boldsymbol{v})}\right)^{-1}((a, b))\right)=\int_{a}^{b} \pi^{(v)}(x) d x+\sum_{x_{j} \in(a, b)} p^{(v)}\left(x_{j}\right),
$$

for any interval $(a, b)$, where $\pi^{(v)}$ is a density function and $p^{(v)}$ is a discrete measure supported on the finite set $\left\{x_{j}\right\}_{j=1}^{m}$. To define $p^{(v)}$ recall that $\Sigma^{\text {gen }}$ has finitely many connected components (Lemma 4.3), denote the connected components on which $\boldsymbol{\rho}^{(\boldsymbol{v})}$ is constant by $\left\{M_{i}\right\}_{i=1}^{m}$ and denote their union by

$$
\Sigma_{\mathrm{disc}}^{\mathrm{gen}}=\cup_{j=1}^{m} M_{j} .
$$

Let $\left\{x_{j}\right\}_{j=1}^{m}$ be the values of $\boldsymbol{\rho}^{(\boldsymbol{v})}$ on $\Sigma_{\text {disc }}^{\text {gen }}$ such that $\left.\boldsymbol{\rho}^{(\boldsymbol{v})}\right|_{M_{i}} \equiv x_{i}$. Note that not all $x_{i}$ are necessarily different. Define a discrete measure on $\mathbb{R}$ by

$$
p^{(v)}:=\sum_{j=1}^{m} \mu_{\vec{l}}\left(M_{j}\right) \delta_{x_{j}}
$$

where $\delta_{x_{j}}$ is the Dirac measure at $x_{j}$. For any Borel set $B \subset \mathbb{R}$,

$$
p^{(v)}(B)=\mu_{\vec{l}}\left(\Sigma_{\operatorname{disc}}^{\text {gen }} \cap\left(\boldsymbol{\rho}^{(\boldsymbol{v})}\right)^{-1}(B)\right) .
$$

Denote the complement $\Sigma_{\text {cont }}^{\text {gen }}=\Sigma^{\text {gen }} \backslash \Sigma_{\text {disc }}^{\text {gen }}$ and define $\zeta^{(v)}:=\boldsymbol{\rho}^{(v)}{ }_{*}\left(\mu_{\vec{l}} \mid \Sigma_{\text {cont }}^{\text {gen }}\right)$ as the pushforward by $\rho^{(v)}$ of the restricted Barra-Gaspard measure $\left.\mu_{\vec{l}}\right|_{\Sigma_{\text {cont }}^{\text {gen }}}$. Namely, for any Borel $B \subset \mathbb{R}$,

$$
\zeta^{(v)}(B)=\mu_{\vec{l}}\left(\Sigma_{\text {cont }}^{\text {gen }} \cap\left(\boldsymbol{\rho}^{(v)}\right)^{-1}(B)\right) .
$$

Summing (7.10) and (7.11) gives that for any Borel $B \subset \mathbb{R}$,

$$
\zeta^{(v)}(B)+p^{(v)}(B)=\mu_{\vec{l}}\left(\left(\boldsymbol{\rho}^{(v)}\right)^{-1}(B)\right) .
$$

We will now show that $\zeta^{(v)}(B)+p^{(v)}(B)$ is a decomposition of a measure to an absolutely continuous part and a discrete part ${ }^{10}$. As $p^{(v)}$ was defined as a discrete measure, we only need to show that that $\zeta^{(v)}$ is absolutely continuous. Once proving that $\zeta^{(v)}$ is absolutely continuous, we may prove $\sqrt{7.7}$ by defining $\pi^{(v)}(x)$ as the Radon-Nykodim derivative of $\zeta^{(v)}$ with respect to the Lebesgue measure, so that we get $d \zeta^{(v)}(x)=\pi^{(v)}(x) d x$.

The measure $\zeta^{(v)}$ is absolutely continuous if for every $B \subset \mathbb{R}$ of Lebesgue measure zero, $\zeta^{(v)}(B)=0$. Let $B \subset \mathbb{R}$ of Lebesgue measure zero and let $M$ be a connected component of $\Sigma_{\text {cont }}^{\text {gen }}$, with the volume element $d \mu$. As $M$ is a connected real analytic manifold and the restriction $\left.\boldsymbol{\rho}^{(v)}\right|_{M}$ is a non-constant real analytic function, we may use Lemma 7.1, (2) to deduce that $\mu\left(\left(\boldsymbol{\rho}^{(\boldsymbol{v})}\right)^{-1}(B) \cap M\right)=0$. Since $\mu$ and the restriction of the Barra-Gaspard

\footnotetext{
${ }^{10}$ Actually, we conjecture that there is no connected component on which $\rho^{(v)}$ is constant, and so $p^{(v)} \equiv 0$. See Remark 7.2 after the proof.
} 
measure $\left.\mu_{\vec{l}}\right|_{M}$ are equivalent, we get that also $\mu_{\vec{l}}\left(\left(\boldsymbol{\rho}^{(\boldsymbol{v})}\right)^{-1}(B) \cap M\right)=0$. As this holds for every connected component of $\Sigma_{\text {cont }}^{\text {gen }}$, taking the union gives

$$
\zeta^{(v)}(B)=\mu_{\vec{l}}\left(\boldsymbol{\rho}^{(\boldsymbol{v})^{-1}}(B) \cap \Sigma_{\mathrm{cont}}^{\mathrm{gen}}\right)=0,
$$

which finishes the first part of the proof.

Next, we prove part (2) of the proposition,

$$
\pi^{(v)}(x)=\pi^{(v)}(\operatorname{deg}(v)-x) \quad \text { and } \quad p^{(v)}(x)=p^{(v)}(\operatorname{deg}(v)-x) .
$$

By 5.8 in Lemma 5.2, we have that $\boldsymbol{\rho}^{(\boldsymbol{v})}(\mathcal{I}(\vec{\kappa}))=\operatorname{deg}(v)-\boldsymbol{\rho}^{(\boldsymbol{v})}(\vec{\kappa})$, where $\mathcal{I}: \vec{\kappa} \mapsto[-\vec{\kappa}]$ is the inversion (see Section 4.3). If $M_{i}$ is a connected component of $\Sigma_{\text {disc }}^{\text {gen }}$, on which $\left.\boldsymbol{\rho}^{(v)}\right|_{M_{i}} \equiv$ $x_{i}$ then $\mathcal{I}\left(M_{i}\right)$ is a connected open subset of $\Sigma^{\text {gen }}$ on which $\left.\boldsymbol{\rho}^{(v)}\right|_{\mathcal{I}\left(M_{i}\right)} \equiv \operatorname{deg}(v)-x_{i}$. It follows $\mathcal{I}\left(M_{i}\right)$ is contained in some $M_{j}$, connected component of $\Sigma_{\text {disc }}^{\text {gen }}$. Since $\mathcal{I}^{2}\left(M_{i}\right)=M_{i}$ then in fact $\mathcal{I}\left(M_{i}\right)=M_{j}$, with $x_{j}=\operatorname{deg}(v)-x_{i}$. This argument shows that $\Sigma_{\text {disc }}^{\text {gen }}$ is $\mathcal{I}$ invariant, and since we know that $\Sigma^{\text {gen }}$ is also $\mathcal{I}$ invariant (Lemma 4.12,(1)) then so does $\Sigma_{\text {cont }}^{\text {gen }}$. Given the relation $\boldsymbol{\rho}^{(\boldsymbol{v})}(\mathcal{I}(\vec{\kappa}))=\operatorname{deg}(v)-\boldsymbol{\rho}^{(\boldsymbol{v})}(\vec{\kappa})$, then for any Borel set $B \subset \mathbb{R}$ and its reciprocal set:

$$
\operatorname{deg}(v)-B:=\{\operatorname{deg}(v)-x: x \in B\}
$$

we have

$$
\begin{aligned}
& \mathcal{I}\left(\Sigma_{\text {disc }}^{\text {gen }} \cap\left(\boldsymbol{\rho}^{(\boldsymbol{v})}\right)^{-1}(B)\right)=\Sigma_{\text {disc }}^{\text {gen }} \cap\left(\boldsymbol{\rho}^{(\boldsymbol{v})}\right)^{-1}(\operatorname{deg}(v)-B), \text { and } \\
& \mathcal{I}\left(\Sigma_{\text {cont }}^{\text {gen }} \cap\left(\boldsymbol{\rho}^{(\boldsymbol{v})}\right)^{-1}(B)\right)=\Sigma_{\text {cont }}^{\text {gen }} \cap\left(\boldsymbol{\rho}^{(\boldsymbol{v})}\right)^{-1}(\operatorname{deg}(v)-B) .
\end{aligned}
$$

The symmetry of both $p^{(v)}$ and $\zeta^{(v)}$ is now a consequence of $\mathcal{I}$ being $\mu_{\vec{l}}$ preserving (Lemma 4.12):

$$
\begin{aligned}
& p^{(v)}(B)=\mu_{\vec{l}}\left(\Sigma_{\text {disc }}^{\text {gen }} \cap\left(\boldsymbol{\rho}^{(\boldsymbol{v})}\right)^{-1}(B)\right)=\mu_{\vec{l}}\left(\Sigma_{\text {disc }}^{\text {gen }} \cap\left(\boldsymbol{\rho}^{(\boldsymbol{v})}\right)^{-1}(\operatorname{deg}(v)-B)\right)=p^{(v)}(\operatorname{deg}(v)-B) \\
& \zeta^{(v)}(B)=\mu_{\vec{l}}\left(\Sigma_{\text {cont }}^{\text {gen }} \cap\left(\boldsymbol{\rho}^{(\boldsymbol{v})}\right)^{-1}(B)\right)=\mu_{\vec{l}}\left(\Sigma_{\text {cont }}^{\text {gen }} \cap\left(\boldsymbol{\rho}^{(\boldsymbol{v})}\right)^{-1}(\operatorname{deg}(v)-B)\right)=\zeta^{(v)}(\operatorname{deg}(v)-B) .
\end{aligned}
$$

Remark 7.2. We conjecture that the probability distribution of the wavelength capacity does not contain a discrete part, namely that $p^{(v)} \equiv 0$. In view of the proof above, this is equivalent to the function $\rho^{(v)}$ not being constant on connected components of $\Sigma^{\text {gen }}$. Apart from an intuition (as demanding $\boldsymbol{\rho}^{(\boldsymbol{v})}$ to be constant is highly restrictive) our conjecture is supported by numerical investigations, which show no atoms for the probability distribution of $\rho^{(v)}$ on various graphs (see discussion in Section 8 and Figure 8.3 .

\section{DisCUSSION AND OPEN PROBLEMS}

This paper presents Neumann domains on metric (quantum) graphs. Neumann domains may be perceived as the counterpart of nodal domains. Nodal domains are sub-graphs which are bounded by the zeros of the eigenfunction, whereas Neumann domains are subgraphs bounded by zeros of the eigenfunction's derivative. This close similarity between nodal domains and Neumann domains calls for a comparison. On one hand there are analogous results for both: bounds and probability distributions of their counts (compare 
Theorem 5.1 for Neumann count with [5, Theorem 2.1] for nodal count). Yet, there are also similar statements which have different incarnations. The nodal count of graphs with disjoint cycles is similar to the Neumann count of $(3,1)$-regular tree graphs - both counts have binomial distributions (compare [5, Theorem 2.3] with Theorem 2.6 here).

A useful viewpoint for comparison between the nodal count and the Neumann count is the perspective of inverse problems. Namely, which information on the underlying graph is stored in the nodal $\backslash$ Neumann count sequence. A partial (but quite satisfying) answer lies in Corollary 2.3. By this corollary, the expected value of the nodal surplus distribution equals $\frac{1}{2} \beta$, where $\beta$ is the first Betti number of the graph; and the expected value of the Neumann nodal surplus distribution equals $\frac{1}{2}(\beta-|\partial \Gamma|)$, where $|\partial \Gamma|$ is the size of the graph's boundary. An easy demonstration of the difference between these two 'pieces' of information may be given in terms of tree graphs. By Corollary 2.3 it is evident that the nodal surplus distribution reveals whether the underlying graph is a tree $(\beta=0)$ or not. Furthermore, all tree graphs have exactly the same nodal count sequence ([45, 1, 43]), which distinguishes them from any non-tree graph [7]. But, this also means that the nodal count sequence does not store any information which allows to distinguish between different trees. In this case, the Neumann surplus sequence is more informative as it predicts $|\partial \Gamma|$, the size of the tree's boundary. Furthermore, we find numerically that there are trees, which share the same boundary size, yet their Neumann surplus distributions are different (though with the same expected value); See Figure 8.1. This raises the additional challenge of exposing which information is stored in the Neumann surplus sequence beyond the value of $\beta-|\partial \Gamma|$.

Having demonstrated the differences between the nodal count and the Neumann count, let us consider also uniting their forces. Namely, what we can infer on the underlying graph if we know both its nodal count sequence and its Neumann count sequence. A simple calculation, taking into account that the minimal degree of an interior vertex is at least three gives

$$
\begin{aligned}
& V \leq 2 \beta+2|\partial \Gamma|-2 \\
& E \leq 3 \beta+2|\partial \Gamma|-3 .
\end{aligned}
$$

The above shows that knowing the values of $\beta$ and $|\partial \Gamma|$ (which are stored in the nodal and Neumann count sequences), bounds the number of vertices and edges and by this restricts the possible candidates to a finite set of graphs. This is a huge progress in solving the inverse problem, as it reduces the infinitely many possible graphs (e.g., in the example of tree graphs mentioned above) to a finite number.

A related inverse problem concerns isospectrality. Isospectral graphs are graphs which share the same eigenvalues. It was conjectured that such graphs would have different nodal count [31], or in other words that nodal count resolves isospectrality ${ }^{11}$. This conjecture in its most general form have been refuted by now in [16, 41, 34, 24]. Yet, given the discussion above, one may ask whether isospectrality is resolved by combining both the nodal count and the Neumann count.

The next step would be to investigate the joint probability distribution of the nodal and Neumann surpluses. See Figure 8.2. This figure shows in particular that the random variables $\sigma$ and $\omega$ are dependent. It is interesting to study the correlation coefficient between both, $\operatorname{corr}(\sigma, \omega):=\frac{\mathbb{E}[(\sigma-\mathbb{E}[\sigma])(\omega-\mathbb{E}[\omega])]}{\sqrt{\mathbb{V}[\sigma] \mathbb{V}[\omega]}}$, where $\mathbb{E}$ and $\mathbb{V}$ indicate the corresponding

\footnotetext{
${ }^{11}$ The scope of the conjecture was actually broader than just for quantum graphs and it was stated also for isospectral manifolds and isospectral discrete graphs.
} 


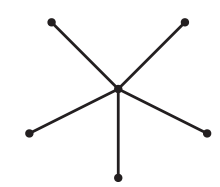

(i) $\Gamma_{1}$

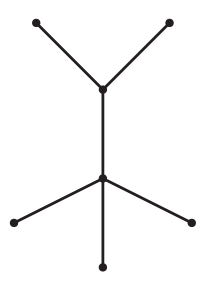

(ii) $\Gamma_{2}$

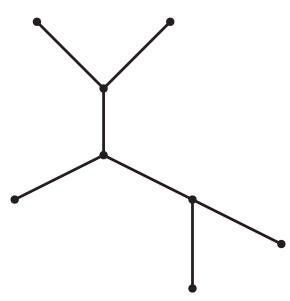

(iii) $\Gamma_{3}$

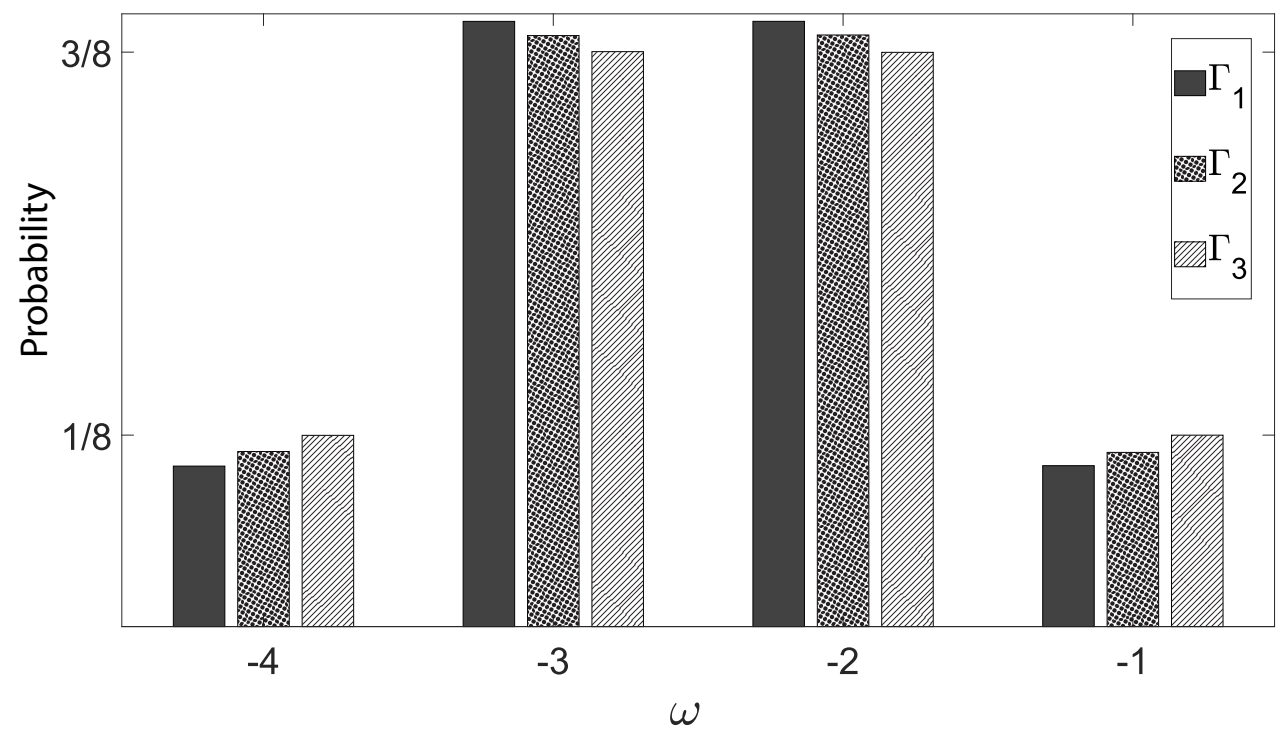

FiguRE 8.1. The Neumann surplus distribution of three different trees with the same boundary size, $|\partial \Gamma|=5$. The expected value of all three probability distributions is the same, but the distributions themselves are different. The numerical data was calculated for $\sim 10^{6}$ eigenfunctions per graph.

expected values and variances. In this context, see also in Appendix A a related discussion on the bounds for $\omega-\sigma$ and for $\omega$.

We end the discussion of the Neumann surplus distribution with

Conjecture 8.1. Let $\left\{\Gamma^{(m)}\right\}_{m=1}^{\infty}$ be a sequence of standard quantum graphs each with rationally independent edge lengths. Denote by $\beta^{(m)}$ and $\left|\partial \Gamma^{(m)}\right|$ the first Betti number and the boundary size of the graph $\Gamma^{(m)}$, correspondingly. Denote by $\omega^{(m)}$ the Neumann surplus random variable of $\Gamma^{(m)}$. If we assume that the graphs in the sequence increase, in the sense $\lim _{m \rightarrow \infty}\left(\beta^{(m)}+\left|\partial \Gamma^{(m)}\right|\right)=\infty$, then

$$
\frac{\omega^{(m)}-\mathbb{E}\left[\omega^{(m)}\right]}{\sqrt{\mathbb{V}\left[\omega^{(m)}\right]}} \underset{m \rightarrow \infty}{\stackrel{\mathcal{D}}{\longrightarrow}} N(0,1),
$$

where the convergence is in distribution and $N(0,1)$ is the standard normal distribution.

Observe that 8.1 describes a convergence of finitely supported distributions, $\frac{\omega^{(m)}-\mathbb{E}\left[\omega^{(m)}\right]}{\sqrt{\mathbb{V}\left[\omega^{(m)}\right]}}$, in to a continuous distribution, $N(0,1)$. This can only happen if the size of the support of $\omega^{(m)}$ is unbounded. The size of the support of $\omega^{(m)}$ is bounded by $3 \beta+|\partial \Gamma|-2$ according to 

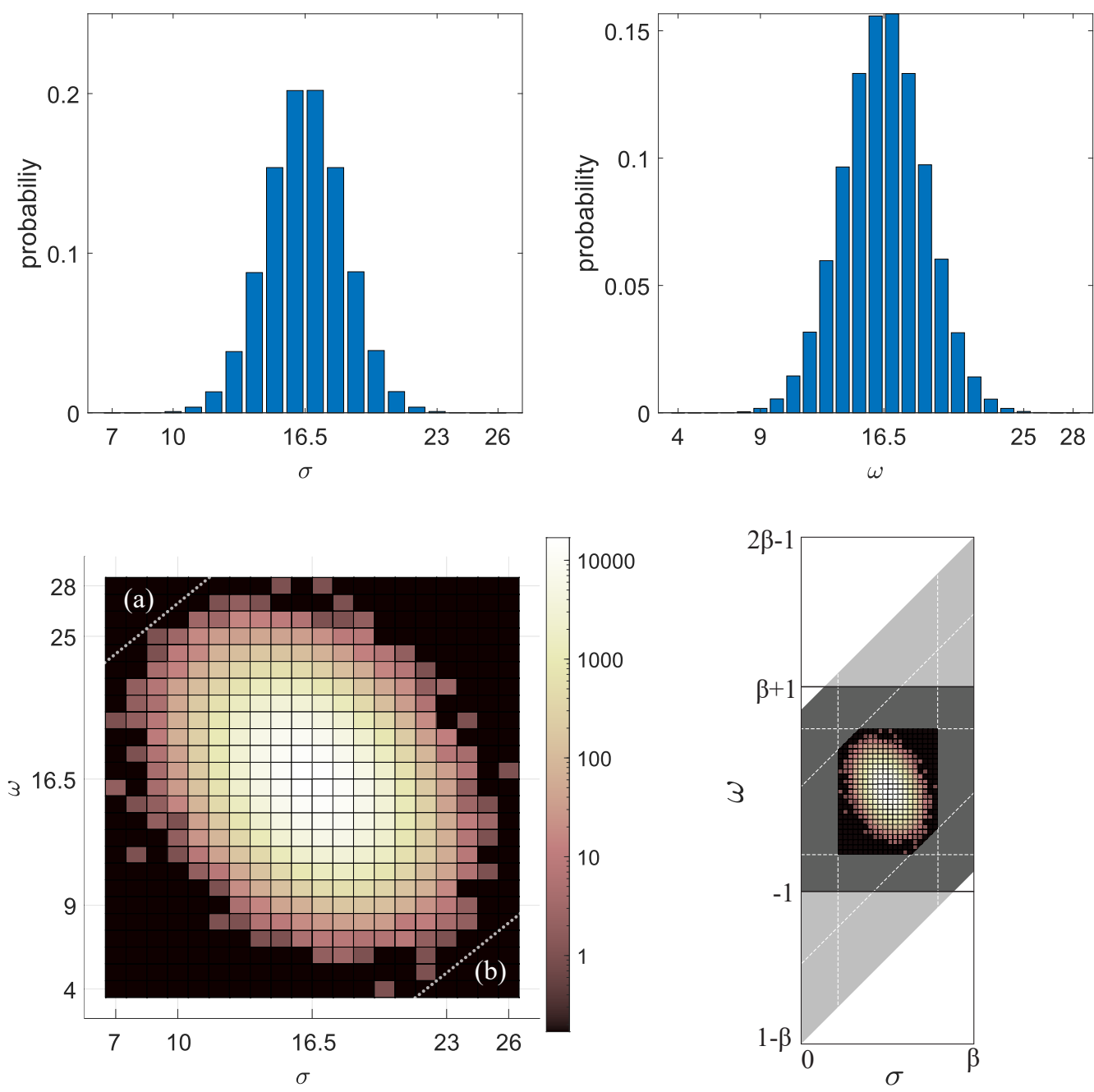

Figure 8.2. The nodal and Neumann statistics for a random 6 regular graph with 16 vertices. The upper two figures show the probability distributions of the nodal surplus, $\sigma$, and the Neumann surplus, $\omega$. The lower left figure shows the joint probability distribution, as a $2 \mathrm{~d}$ histogram of the pair $(\sigma, \omega)$ in a log scale. The support of $(\sigma, \omega)$ in this example is bounded by $7 \leq \sigma \leq 26,4 \leq \omega \leq 28$ and $-16 \leq \sigma-\omega \leq 17$. The white diagonal lines (a) and (b) in the lower left figure represent the support of $\sigma-\omega$. In the lower right figure, the support of $(\sigma, \omega)$ is presented with respect to the bounds on $\omega$ and $\sigma-\omega$. The region which contains the allowed values for $\sigma-\omega$ is shaded with gray colors; light gray is for the $\omega$ bounds of Theorem 2.2 (1) and dark gray for Conjecture 2.4. The numerical data was calculated for $\sim 10^{6}$ eigenfunctions.

Theorem 2.2 (1), and by $\beta+|\partial \Gamma|+2$ if Conjecture 2.4 is true. Both bounds diverge if and only if $\beta+|\partial \Gamma|$ diverges, which is why we consider the $\operatorname{limit} \lim _{m \rightarrow \infty}\left(\beta^{(m)}+\left|\partial \Gamma^{(m)}\right|\right)=\infty$, in the conjecture. 
Note that by Corollary 2.3 it is known that $\mathbb{E}\left[\omega^{(m)}\right]=\frac{1}{2}\left(\beta^{(m)}-\left|\partial \Gamma^{(m)}\right|\right)$, but we do not have a general expression for the variance. There are a few sources of support towards this conjecture. First, by Theorem 2.6, we get that the conjecture holds for an increasing family of $(3,1)$-regular tree graphs. Indeed, the Neumann surplus of each such graph is binomially distributed, so that the central limit theorem guarantees the convergence to the normal distribution, as in the conjecture. Furthermore, we tested the validity of this conjecture by various numerical explorations. We have checked increasing families of graphs such as random d-regular graphs and complete graphs. For each such family we have calculated the Kolmogorov-Smirnov distance from a normal distribution and observed that this distance converges to zero in the limit of increasing graphs. We also provide an analytic evidence to the above conjecture in an ongoing work, where we prove that families of stowers and mandarins (see Appendix A) satisfy the above conjecture. Finally, both authors together with Berkolaiko conjecture a similar statement for the nodal surplus. See 4] for the exact details, as well as supportive evidence. We believe that the work on these two conjectures should be done in parallel and that their confirmation might occur simultaneously.

We dedicate the last part of the discussion to the role of the local observables; the spectral position and the wavelength capacity. Those serve as a fundamental tool for studying Neumann domains. Analogous observables are defined for Neumann domains on manifolds [11, 12, 13, 14. For manifolds, estimating the spectral position may serve as a tool towards calculating the asymptotics of the Neumann count. But, determining the spectral position of a Neumann domain on a manifold is a hard task (even numerically). To aid this, it was shown in [6] that the wavelength capacity ${ }^{12}$ is bounded from above in terms of the spectral position. Hence, computation of the former allows to estimate the latter and tackle the problem mentioned above. In the current paper we show that even a stronger connection exists for quantum graphs - bounds from both sides of the wavelength capacity are expressed in terms of the spectral position.

Taking the perspective of inverse problems, we consider the probability distributions of the wavelength capacity, $\pi^{(v)}$ and $p^{(v)}$, as described in Proposition 2.12. We ask what information on the graph is stored in those distributions. First, as those distributions are symmetric around $\frac{1}{2} \operatorname{deg}(v)$, the degree of that particular vertex is revealed. But, those distributions obviously do not solely depend on $\operatorname{deg}(v)$, as is demonstrated in Figure 8.3 .

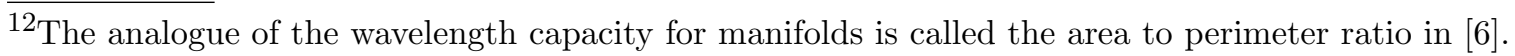



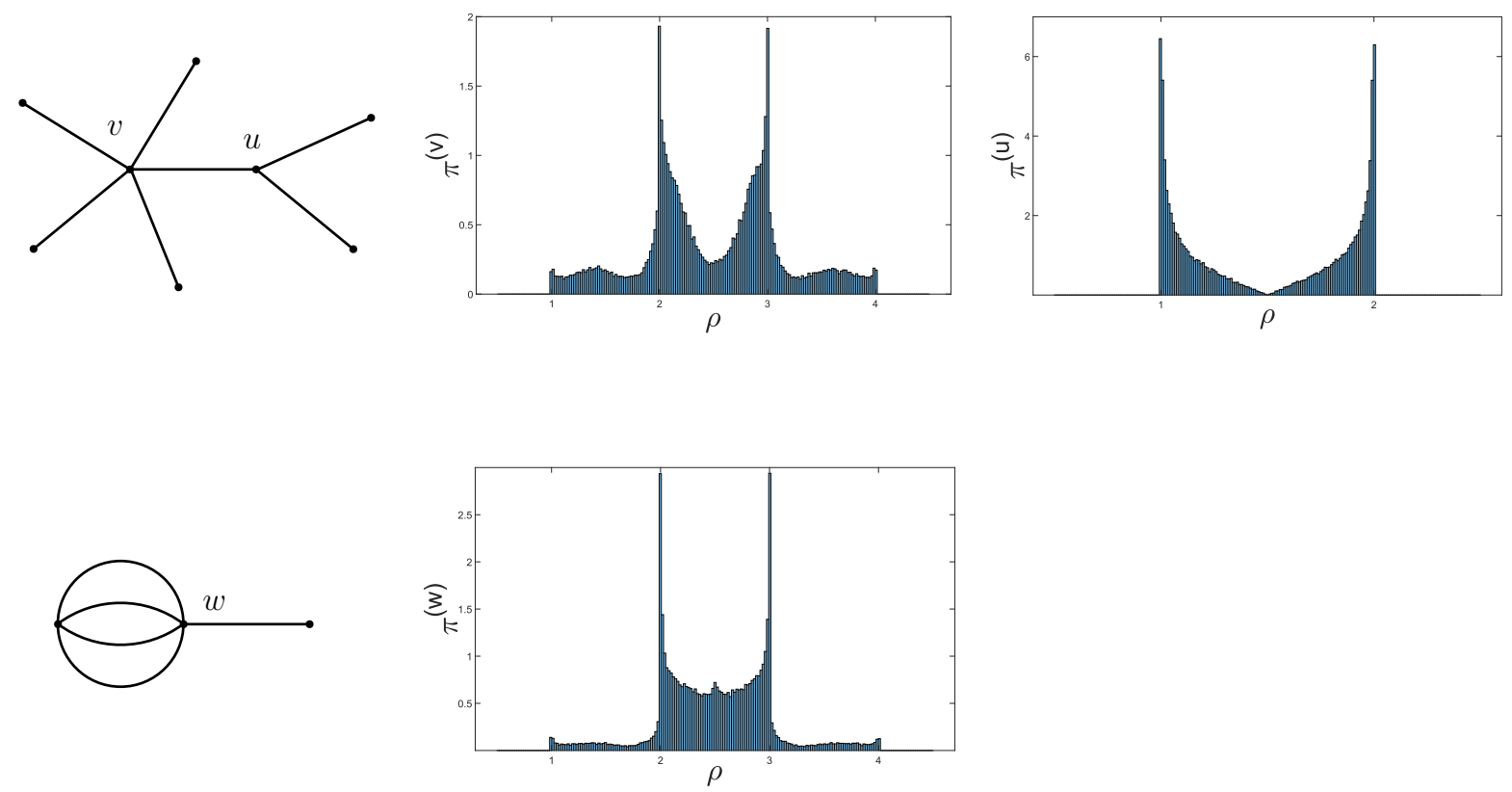

FIGURE 8.3. The top line shows a graph with two marked vertices $v$ and $u$ such that $\operatorname{deg}(v)=5$ and $\operatorname{deg}(u)=3$. Next to the graph are the probability distributions $\pi^{(v)}$ and $\pi^{(u)}$. The bottom line shows a different graph with a marked vertex $w$ of $\operatorname{deg}(w)=5$ and the probability distribution $\pi^{(w)}$. The numerical data was calculated for $\sim 10^{6}$ eigenfunctions per graph.

A stimulating problem would be to understand better the probability distribution $\pi^{(v)}$ and the information it stores on the graph. In particular, the exact values at which it gets minima and maxima (see Figure 8.3) are yet unknown. Another numerical observation is that the probability distribution of the wavelength capacity contains no atoms. Namely, $p^{(v)} \equiv 0$. We conjecture that this is always the case - see Remark 7.2 .

Acknowledgments. We acknowledge Michael Bersudsky and Sebastian Egger for their permission to split this work from the review paper [6] co-authored with them and for the discussions in the course of the common work. We thank Ron Rosenthal and Uri Shapira for the interesting discussions throughout the work. We thank Adi Alon for her graphical assistance with the figures.

The authors were supported by ISF (Grant No. 844/19) and by the Binational Science Foundation Grant (Grant No. 2016281). LA was also supported by the Ambrose Monell Foundation and the Institute for Advanced Study.

\section{Appendix A. Nodal and Neumann surpluses of particular examples}

An intensive numerical investigation led us to believe that the Neumann surplus bounds in Theorem 2.2 are not optimal and to propose better bounds in Conjecture 2.4. In this appendix we provide analytic evidence supporting the conjectured bounds, by calculating the support of the Neumann surplus for three families of graphs:

(1) Stowers - Proposition A.1.

(2) Mandarins - Proposition A.5.

(3) Trees - Proposition A.11. 
See Figure A.1 for examples of a stower and a mandarin. Using the support of the stowers we deduce that the bounds on $\sigma-\omega$, as presented in (3.1), are optimal in terms of $\beta$ and $|\partial \Gamma|$.
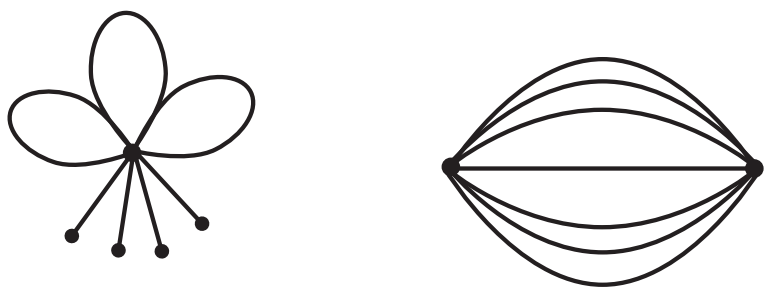

Figure A.1. On the left, a stower graph with $n=3$ loops and $m=4$ tails.

On the right, a mandarin graph with $E=7$ edges.

A.1. Stowers. We say that $\Gamma$ is an $(n, m)$-stower graph with $n$ loops (petals) and $m$ tails (leaves), if it has only one interior vertex $v$ (the central vertex), $m$ boundary vertices, each connected to the central vertex, and $n$ loops connecting the central vertex to itself. See figure A.1 for example. In such case the first Betti number is $\beta=n$ and the boundary size is $|\partial \Gamma|=m$.

Proposition A.1. Let $\Gamma$ be a stower, then its Neumann surplus $\omega$ is bounded by

$$
1-|\partial \Gamma| \leq \omega \leq \beta-1 .
$$

Moreover, if $\Gamma$ has rationally independent edge lengths then its Neumann surplus and nodal surplus distributions satisfy

$$
\begin{aligned}
\forall j \in\{1-|\partial \Gamma|, \ldots, \beta-1\} & P(\omega=j)>0 \\
\forall j \in\{1-\beta, \ldots, \beta+|\partial \Gamma|-1\} & P(\sigma-\omega=j)>0,
\end{aligned}
$$

and if $|\partial \Gamma|>0$, then

$$
\forall j \in\{0, \ldots, \beta\} \quad P(\sigma=j)>0 .
$$

In the case of $|\partial \Gamma|=0$, namely $\Gamma$ is a flower graph, the nodal surplus us bounded by

$$
1 \leq \sigma \leq \beta-1
$$

and

$$
\forall j \in\{1, \ldots, \beta-1\} \quad P(\sigma=j)>0 .
$$

This result shows that for any possible choice of $\beta$ and $|\partial \Gamma|$ there is a corresponding stower graph such that its $\sigma-\omega$ sequence achieves both upper and lower bounds in (3.1). This result supports Conjecture 2.4 and shows that the bounds in (3.1) are optimal.

To prove Proposition A.1 let us first state the following.

Definition A.2. Let $\Gamma$ be a stower with $m$ tails and $n$ loops. For distinction, denote points in $\mathbb{T}^{E}$ by $(\vec{y}, \vec{z})$ such that $\vec{y} \in \mathbb{T}^{n}$ corresponds to loops and $\vec{z} \in \mathbb{T}^{m}$ corresponds to tails. Call a tail coordinate $z_{j}$ "bad" if either $\sin \left(z_{j}\right)=0$ or $\cos \left(z_{j}\right)=0$. Similarly, a loop coordinate $y_{i}$ is "bad" if either $\sin \left(\frac{y_{i}}{2}\right)=0$ or $\cos \left(\frac{y_{i}}{2}\right)=0$.

Denote the set of points in $\mathbb{T}^{E}$ which have at least one "bad" coordinate by $\boldsymbol{B}^{(1)}$, and the similarly denote the set of points with at least two "bad" coordinates by $\boldsymbol{B}^{(2)}$. 
Remark A.3. Notice that $\operatorname{dim}\left(\boldsymbol{B}^{(j)}\right)=E-j$.

Lemma A.4. Let $\Gamma$ be a stower with $m$ tails and $n$ loops and consider the notations of Definition (A.2). Then,

(1) $\Sigma^{\text {gen }}$ is given by

$$
\Sigma^{\text {gen }}=\left\{(\vec{y}, \vec{z}) \in \mathbb{T}^{E} \backslash \boldsymbol{B}^{(1)}: \sum_{j=1}^{m} \tan \left(z_{j}\right)+2 \sum_{i=1}^{n} \tan \left(\frac{y_{i}}{2}\right)=0\right\} .
$$

(2) We denote for $(\vec{y}, \vec{z}) \in \Sigma^{\text {gen }}$

$$
\begin{aligned}
\boldsymbol{i}_{\text {tails }}(\vec{z}) & :=\left|\left\{1 \leq j \leq m: \tan \left(z_{j}\right)<0\right\}\right|, \\
\boldsymbol{i}_{\text {loops }}(\vec{y}) & :=\left|\left\{1 \leq i \leq n: \tan \left(\frac{y_{i}}{2}\right)<0\right\}\right| .
\end{aligned}
$$

Then the Neumann surplus and nodal surplus functions (introduced in Lemma 5.2 satisfy,

$$
\begin{aligned}
& \boldsymbol{\omega}(\vec{y}, \vec{z})=n-\left(\boldsymbol{i}_{\text {tails }}(\vec{z})+\boldsymbol{i}_{\text {loops }}(\vec{y})\right), \\
& \boldsymbol{\sigma}(\vec{y}, \vec{z})=\boldsymbol{i}_{\text {loops }}(\vec{y}) .
\end{aligned}
$$

The proof of Lemma A.4 appears after the proof of Proposition A.1.

Proof of Proposition A.1. According to Theorem 2.2 (2b), Theorem 4.8 and Lemma 5.2. it is enough to prove that the functions $\boldsymbol{\sigma}$ and $\boldsymbol{\omega}$, defined on $\Sigma^{\text {gen }}$, satisfy

$$
\begin{aligned}
\operatorname{Image}(\boldsymbol{\omega}) & =\{1-|\partial \Gamma|, \ldots, \beta-1\}, \\
\operatorname{Image}(\boldsymbol{\sigma}-\boldsymbol{\omega}) & =\{1-\beta, \ldots, \beta+|\partial \Gamma|-1\},
\end{aligned}
$$

and

$$
\operatorname{Image}(\boldsymbol{\sigma})=\left\{\begin{array}{ll}
\{0, \ldots \beta\} & |\partial \Gamma|>0 \\
\{1, \ldots \beta-1\} & |\partial \Gamma|=0
\end{array} .\right.
$$

Let $\Gamma$ be a stower with $m$ tails and $n$ loops and consider the notations of Definition (A.2). For every point $(\vec{y}, \vec{z}) \in \mathbb{T}^{E}$, define $t_{j}, s_{i} \in \mathbb{R} \cup\{\infty\}$ by $t_{j}:=\tan \left(z_{j}\right)$ and $s_{i}:=\tan \left(\frac{y_{i}}{2}\right)$ for all coordinates $z_{j}$ and $y_{i}$. Notice that $(\vec{y}, \vec{z}) \in \mathbb{T}^{E} \backslash \boldsymbol{B}^{(1)}$ if and only if all $t_{j}$ 's and $s_{j}$ ' lie in $\mathbb{R} \backslash\{0\}$. According to Lemma A.4 (1), $(\vec{y}, \vec{z}) \in \Sigma^{\text {gen }}$ if and only if $(\vec{y}, \vec{z}) \in \mathbb{T}^{E} \backslash \boldsymbol{B}^{(1)}$ and

$$
\sum_{j=1}^{m} t_{j}+2 \sum_{j=1+m}^{m+n} s_{j}=0
$$

The $\boldsymbol{i}_{\text {tails }}(\vec{z})$ and $\boldsymbol{i}_{\text {loops }}(\vec{y})$ indices, defined in Lemma A.4 (2), are equal to the number of negative $t_{j}$ 's and $s_{j}$ 's correspondingly. Observe that

$$
\begin{aligned}
& 0 \leq \boldsymbol{i}_{\text {tails }} \leq m, \text { and } \\
& 0 \leq \boldsymbol{i}_{\text {loops }} \leq n,
\end{aligned}
$$

however a solution to A.9 with non-zero $t_{j}$ 's and $s_{j}$ ' must have at least one positive and one negative summands, and therefore

$$
1 \leq \boldsymbol{i}_{\text {tails }}+\boldsymbol{i}_{\text {loops }} \leq m+n-1 .
$$

Using Lemma A.4 (2) and the bounds in A.10, A.11 and (A.12), proves the corresponding bounds on $\boldsymbol{\omega}, \boldsymbol{\sigma}$ and $\boldsymbol{\sigma}-\boldsymbol{\omega}$ and hence inclusion in A.6, A.7) and A.8. 
In order to prove the actual equalities in $(\mathrm{A.6}),(\mathrm{A} .7)$ and $(\mathrm{A.8})$, namely that every value is attained, we show that for any integers $i_{t}$ and $i_{s}$ satisfying

$$
\begin{aligned}
& 0 \leq i_{t} \leq m, \\
& 0 \leq i_{s} \leq n, \text { and } \\
& 1 \leq i_{t}+i_{s} \leq m+n-1,
\end{aligned}
$$

there exist $(\vec{y}, \vec{z}) \in \Sigma^{\text {gen }}$ for which $\boldsymbol{i}_{\text {tails }}(\vec{z})=i_{t}$ and $\boldsymbol{i}_{\text {loops }}(\vec{y})=i_{s}$. Clearly, one can construct a solution to $\mathrm{A} .9$ for which all $t_{j}$ 's and $s_{j}$ ' lie in $\mathbb{R} \backslash\{0\}$ and there are exactly $i_{t}$ negative $t_{j}$ 's and $i_{s}$ negative $s_{j}$ 's. Consider $\tan ^{-1}: \mathbb{R} \backslash\{0\} \rightarrow\left(0, \frac{\pi}{2}\right) \cup\left(\frac{\pi}{2}, \pi\right)$ and define $z_{j}=\tan ^{-1}\left(t_{j}\right)$ for all $j \leq m$ and $y_{i}=2 \tan ^{-1}\left(s_{i}\right)$ for $1 \leq i \leq n$. By construction, $(\vec{y}, \vec{z}) \in \Sigma^{\text {gen }}$ and satisfies $\boldsymbol{i}_{\text {tails }}(\vec{z})=i_{t}$ and $\boldsymbol{i}_{\text {loops }}(\vec{y})=i_{s}$.

It remains to prove Lemma A.4.

Proof of Lemma A.4. The first part of the lemma is an explicit characterization $\Sigma^{\text {gen }}$. To do so consider the standard graph $\Gamma_{(\vec{y}, \vec{z})}$, i.e., with edge lengths $\vec{l}=(\vec{y}, \vec{z})$. In the following, we construct an eigenfunction $f$ of $\Gamma_{\vec{\kappa}}$ with eigenvalue $k=1$, providing the conditions on $(\vec{y}, \vec{z}) \in \mathbb{T}^{E}$ for which such an eigenfunction exists.

Let $v$ be the central vertex of $\Gamma$, and consider a parametrization of each tail $e_{j}$ by arclength parametrization $x_{j} \in\left[0, z_{j}\right]$ with $x_{j}=0$ at $v$. Consider a similar parametrization for each loop $e_{i}$ but let $x_{i} \in\left[-\frac{y_{i}}{2}, \frac{y_{i}}{2}\right]$ such that $x_{i}= \pm \frac{y_{i}}{2}$ at $v$. Let $f$ be an eigenfunction of eigenvalue $k=1$, then its restriction to every tail $e_{j}$ can be written as:

$$
\left.f\right|_{e_{j}}\left(x_{j}\right)=A_{j} \cos \left(z_{j}-x_{j}\right),
$$

and its restriction to every loop $e_{i}$ can be written as:

$$
\left.f\right|_{e_{i}}\left(x_{i}\right)=C_{i} \cos \left(x_{i}\right)+B_{i} \sin \left(x_{i}\right) .
$$

For every loop $e_{i}$, consider the inversion defined by $x_{i} \mapsto-x_{i}$ on $e_{i}$ while fixing $\Gamma \backslash e_{i}$. It is an isometry of $\Gamma_{(\vec{y}, \vec{z})}$ and so we may choose all eigenfunctions of $\Gamma_{(\vec{y}, \vec{z})}$ to be either symmetric or anti-symmetric with respect to this inversion. Anti-symmetric eigenfunctions are loop-eigenfunctions (as defined in Subsection 1.3) and are not generic eigenfunctions (in the sense of Definition 1.3). A loop-eigenfunction which is supported on $e_{i}$ exists if and only if $y_{i}=2 \pi$, in which case $(\vec{y}, \vec{z}) \in \boldsymbol{B}^{(1)}$.

We call $f$ symmetric if it is symmetric on every loop. If $f$ is symmetric then all $B_{i}$ 's in A.14 are zero. For symmetric eigenfunctions, the Neumann vertex condition at $v$ can be written as,

$$
\begin{array}{r}
\forall j \leq m \quad A_{j} \cos \left(z_{j}\right)=f(v), \\
\forall i \leq n \quad C_{i} \cos \left(\frac{y_{i}}{2}\right)=f(v), \text { and } \\
-k\left(\sum_{j=1}^{m} A_{j} \sin \left(z_{j}\right)+2 \sum_{i=1}^{m} C_{i} \sin \left(\frac{y_{i}}{2}\right)\right)=0 .
\end{array}
$$

In particular, for A.17 to hold there must be at least two non zero amplitudes among all $A_{j}$ 's and $C_{i}$ 's. We may deduce from A.15 and A.16 that if $f$ is symmetric with $f(v)=0$, then $(\vec{y}, \vec{z}) \in \boldsymbol{B}^{(2)}$. 
A symmetric eigenfunction $f$, with $f(v) \neq 0$, implies that $\cos \left(z_{j}\right) \neq 0$ for every tail and $A_{j}=\frac{f(v)}{\cos \left(z_{j}\right)}$ by A.15. Similarly, for every loop, $\cos \left(\frac{y_{i}}{2}\right) \neq 0$ and $C_{i}=\frac{f(v)}{\cos \left(\frac{y_{i}}{2}\right)}$ by A.16. Dividing A.17 by $-k f(v) \neq 0$, gives

$$
\sum_{j=1}^{m} \tan \left(x_{j}\right)+2 \sum_{i=1}^{n} \tan \left(\frac{y_{i}}{2}\right)=0 .
$$

In such case, the outgoing derivatives of $f$ at $v$ do not vanish if and only if,

$$
\forall j \leq m \sin \left(z_{j}\right) \neq 0 \text { and } \forall 1 \leq i \leq n \sin \left(\frac{y_{i}}{2}\right) \neq 0 .
$$

We may now deduce that $f$ is not generic if $(\vec{y}, \vec{z}) \in \boldsymbol{B}^{(1)}$. Moreover, if $(\vec{y}, \vec{z}) \notin \boldsymbol{B}^{(1)}$ then $\Gamma_{(\vec{y}, \vec{z})}$ has an eigenfunction $f$ of eigenvalue $k=1$ if and only if A.18) holds, and in such case the construction implies that $f$ is unique (up to a scalar multiplication), $f(v) \neq 0$, and non of the outgoing derivatives of $f$ at $v$ vanish. Namely, $f$ is generic. This proves Lemma A.4 (1), that is:

$$
\Sigma^{\mathrm{gen}}=\left\{(\vec{z}, \vec{y}) \in \mathbb{T}^{E} \backslash \boldsymbol{B}^{(1)}: \sum_{j=1}^{m} \tan \left(x_{j}\right)+2 \sum_{i=1}^{n} \tan \left(\frac{y_{i}}{2}\right)=0\right\} .
$$

To prove Lemma A.4 (2), let $(\vec{y}, \vec{z}) \in \Sigma^{\text {gen }}$ and let $f$ be the corresponding generic eigenfunction of eigenvalue $k=1$. Since $f$ must be symmetric, all $B_{i}$ 's in are zero, and we may derive the nodal and Neumann counts from (A.13) and (A.14):

$$
\begin{aligned}
& \phi(f)=\left|\left\{j \leq m: z_{j}>\frac{\pi}{2}\right\}\right|+\left|\left\{j \leq m: z_{j}>\frac{3 \pi}{2}\right\}\right|+\ldots \\
& \quad \ldots+2\left|\left\{1 \leq i \leq n: y_{i}>\pi\right\}\right| \\
& \xi(f)=\left|\left\{j \leq m: z_{j}>\pi\right\}\right|+n .
\end{aligned}
$$

The nodal and Neumann surpluses are given by subtracting the spectral position $N(\vec{z}, \vec{y})$ from the above. The spectral position is defined by,

$$
\begin{aligned}
N(\vec{y}, \vec{z}): & :=\mid\left\{k \in(0,1]: \quad k \text { is an eigenvalue of } \Gamma_{(\vec{y}, \vec{z})}\right\} \mid, \\
& =|\{t \in(0,1]:(t \vec{y}, t \vec{z}) \in \Sigma\}|,
\end{aligned}
$$

both counts including multiplicity. In order to avoid multiplicities and to ease the computations define the "good" set:

$$
M=\left\{(\vec{y}, \vec{z}) \in \Sigma^{\text {gen }}: \forall t \in(0,1] \quad(t \vec{y}, t \vec{z}) \notin \boldsymbol{B}^{(2)} \cup \Sigma^{\text {sing }}\right\} .
$$

Let $(\vec{y}, \vec{z}) \in M$ and let $t \in(0,1)$ such that $(t \vec{y}, t \vec{z}) \in \Sigma$, then $(t \vec{y}, t \vec{z}) \in \Sigma^{\mathrm{reg}}$ and corresponds to an eigenfunction that satisfies $f(v) \neq 0$ since $(t \vec{y}, t \vec{z}) \notin \boldsymbol{B}^{(2)}$ and $t y_{i}<2 \pi$ for all $i \leq n$. Therefore, $N(\vec{y}, \vec{z})$ for $(\vec{y}, \vec{z}) \in M$ is given by

$$
N(\vec{y}, \vec{z})=\left|\left\{t \in(0,1]: \sum_{j=1}^{m} \tan \left(t z_{j}\right)+2 \sum_{i=1}^{n} \tan \left(t \frac{y_{i}}{2}\right)=0\right\}\right| .
$$

Define $F_{(\vec{y}, \vec{z})}:(0,1] \rightarrow \mathbb{R} \cup\{\infty\}$ by

$$
F_{(\vec{y}, \vec{z})}(t)=\sum_{j=1}^{m} \tan \left(t z_{j}\right)+2 \sum_{i=1}^{n} \tan \left(t \frac{y_{i}}{2}\right) \text {. }
$$


If $(\vec{y}, \vec{z}) \in M$ then only one of the summands of $F_{(\vec{y}, \vec{z})}(t)$ can diverge at each time $t \in(0,1]$ since $(t \vec{y}, t \vec{z}) \notin \boldsymbol{B}^{(2)}$. Therefore, the poles of $F_{(\vec{y}, \vec{z})}(t)$ are simple. Since $\frac{d}{d t} F_{(\vec{y}, \vec{z})}(t)>0$ whenever $t$ is not a pole then the zeros of $F_{(\vec{y}, \vec{z})}$ are simple and they interlace with the poles. Since the interlacing sequence of zeros and poles ends with a zero at $F_{(\vec{y}, \vec{z})}(1)=0$, we can find $N(\vec{y}, \vec{z})$ by counting poles:

$$
\begin{aligned}
N(\vec{y}, \vec{z}) & =\left|\left\{j \leq m: z_{j}>\frac{\pi}{2}\right\}\right|+\left|\left\{j \leq m: z_{j}>\frac{3 \pi}{2}\right\}\right|+\ldots \\
\ldots+ & +\left\{1 \leq i \leq n: y_{i}>\pi\right\} \mid .
\end{aligned}
$$

Subtracting $N(\vec{y}, \vec{z})$ from A.21 and A.23) gives the nodal and Neumann surplus for any $(\vec{y}, \vec{z}) \in M:$

$$
\begin{aligned}
& \boldsymbol{\omega}(\vec{y}, \vec{z})=n+\left|\left\{j \leq m: z_{j}>\pi\right\}\right|-N(\vec{z}, \vec{y}), \\
& \boldsymbol{\sigma}(\vec{y}, \vec{z})=\left|\left\{1 \leq i \leq n: y_{i}>\pi\right\}\right|,
\end{aligned}
$$

which can be rewritten, using $\boldsymbol{i}_{\text {tails }}$ and $\boldsymbol{i}_{\text {loops }}$ (as defined in Lemma A.4 (2)), by

$$
\begin{aligned}
& \boldsymbol{\omega}(\vec{y}, \vec{z})=n-\left(\boldsymbol{i}_{\text {tails }}(\vec{z})-\boldsymbol{i}_{\text {loops }}(\vec{y})\right) . \\
& \boldsymbol{\sigma}(\vec{y}, \vec{z})=\boldsymbol{i}_{\text {loops }}(\vec{y}) .
\end{aligned}
$$

This proves the statement of Lemma A.4 (2) for points in $M$.

In order to extend this result from $M$ to $\Sigma^{\text {gen }}$ we recall that $\boldsymbol{\sigma}$ and $\boldsymbol{\omega}$ are constant on each connected component of $\Sigma^{\text {gen }}$ (Lemma 5.2 and Theorem 5.1). Observe that $\boldsymbol{i}_{\text {tails }}$ and $\boldsymbol{i}_{\text {loops }}$ are constant on connected components of $\mathbb{T}^{E} \backslash \boldsymbol{B}^{(1)}$ and are therefore constant on connected components of $\Sigma^{\text {gen }} \subset \mathbb{T}^{E} \backslash \boldsymbol{B}^{(1)}$ as well. It is thus enough to show that $M$ intersects each of the connected components of $\Sigma^{\text {gen }}$ to conclude that Lemma A.4 (2) holds. To do so, we prove that $M$ is dense in $\Sigma^{\text {gen }}$.

Consider the open set $O=\Sigma^{\text {gen }} \backslash \bar{M}$ so that we need to prove that $O=\emptyset$. Consider the cone $P O:=\{(t \vec{y}, t \vec{z}): t \in(0,1],(\vec{y}, \vec{z}) \in O\}$. By the definition of $P O$, it is a union of lines of the form $\{(t \vec{y}, t \vec{z}): t \in(0,1]\}$ for some $(\vec{y}, \vec{z}) \in O$. Each such line intersect $\boldsymbol{B}^{(2)} \cup \Sigma^{\text {sing }}$, by the definition of $O$, and each two such lines are either disjoint or one is contained in the other. It follows that the dimension of $P O$ is bounded by

$$
\operatorname{dim}(P O) \leq \operatorname{dim}\left(\boldsymbol{B}^{(2)} \cup \Sigma^{\text {sing }}\right)+1=E-1 .
$$

In the last equality we use Remark A.3 which states that $\operatorname{dim}\left(\boldsymbol{B}^{(2)}\right)=E-2$ and the fact that $\operatorname{dim}\left(\Sigma^{\mathrm{sing}}\right) \leq E-2$. On the other hand, by Lemma A.4 11],

$$
\Sigma^{\text {gen }}=\left\{(\vec{y}, \vec{z}) \in \mathbb{T}^{E} \backslash \boldsymbol{B}^{(1)}: F_{(\vec{y}, \vec{z})}(1)=0\right\},
$$

and since $\left.\frac{d}{d t} F_{(\vec{y}, \vec{z})}(t)\right|_{t=1}>0$ on every $(\vec{y}, \vec{z}) \in \Sigma^{\text {gen }}$, then each of the lines in $P O$ intersect $\Sigma^{\text {gen }}$ transversely. It follows that

$$
\operatorname{dim}(P O)=\operatorname{dim}(O)+1 .
$$

It follows from $(\mathrm{A} .29)$ that $\operatorname{dim}(O) \leq E-2$. However, $O$ is open in $\Sigma^{\text {gen }}$ which is of $\operatorname{dim} \Sigma^{\text {gen }}=E-1$ so $O=\emptyset$. Therefore, $M$ is dense in $\Sigma^{\text {gen }}$. 
A.2. Mandarins. A mandarin graph $\Gamma$ is a graph with only two vertices and $E \geq 3$ edges such that every edge is connected to both vertices. See figure A.1 for example. In such case $\beta=E-1$ and $\partial \Gamma=\emptyset$.

Proposition A.5. If $\Gamma$ is a mandarin then its Neumann surplus is bounded by

$$
0 \leq \omega \leq \beta,
$$

its nodal surplus is bounded by

$$
1 \leq \sigma \leq \beta-1,
$$

and the difference $\sigma-\omega$ is supported inside the set $\{1-\beta, 3-\beta, 5-\beta, \ldots,-1+\beta\}$.

Moreover, if $\Gamma$ has rationally independent edge lengths, then its nodal surplus and Neumann surplus distributions satisfy

$$
\begin{aligned}
\forall j \in\{0,1, \ldots \beta-1\} & P(\omega=j)+P(\omega=j+1)>0, \\
\forall j \in\{1, \ldots \beta-2\} & P(\sigma=j)+P(\sigma=j+1)>0, \text { and } \\
\forall j \in\{1-\beta, 3-\beta, 5-\beta, \ldots,-1+\beta\} & P(\sigma-\omega=j)>0 .
\end{aligned}
$$

This example supports Conjecture 2.4 and shows that the bounds in (3.1) can be achieved. To prove Proposition A.5 let us first state the following.

Remark A.6. The bound $1 \leq \sigma \leq \beta-1$ for mandarin graphs was already shown in [10].

Definition A.7. Let $\Gamma$ be a mandarin graph with $E$ edges. Call a coordinate $\kappa_{j}$ "bad" if either $\sin \left(\frac{\kappa_{j}}{2}\right)=0$ or $\cos \left(\frac{\kappa_{j}}{2}\right)=0$. Denote the set of points in $\mathbb{T}^{E}$ which have at least one "bad" coordinate by $\boldsymbol{B}^{(1)}$, and the similarly denote the set of points with at least two "bad" coordinates by $\boldsymbol{B}^{(2)}$.

Lemma A.8. Let $\Gamma$ be a mandarin with $E$ edges. Define two functions on $\mathbb{T}^{E}$ by,

$$
\begin{aligned}
& F_{s}(\vec{\kappa}):=\sum_{j=1}^{E} \tan \left(\frac{\kappa_{j}}{2}\right), \text { and } \\
& F_{a}(\vec{\kappa}):=\sum_{j=1}^{E} \cot \left(\frac{\kappa_{j}}{2}\right) .
\end{aligned}
$$

Then $\Sigma^{\text {gen }}$ has a disjoint decomposition $\Sigma^{\text {gen }}=\Sigma_{s}^{\text {gen }} \sqcup \Sigma_{a}^{\text {gen }}$ such that,

(1) $\sum_{s}^{\text {gen }}$ is given by

$$
\Sigma_{s}^{\text {gen }}=\left\{\vec{\kappa} \in \mathbb{T}^{E} \backslash B^{(1)}: F_{s}(\vec{\kappa})=0 \text {, and } F_{a}(\vec{k}) \neq 0\right\},
$$

and $\Sigma_{a}^{\text {gen }}$ is given by

$$
\Sigma_{s}^{\text {gen }}=\left\{\vec{\kappa} \in \mathbb{T}^{E} \backslash \boldsymbol{B}^{(1)}: F_{s}(\vec{\kappa}) \neq 0 \text {, and } F_{a}(\vec{\kappa})=0\right\} .
$$

In particular, the inversion $T(\vec{\kappa}):=[\vec{\kappa}+(\pi, \pi, \pi \ldots)$.$] , is an isometry between the$ two components, $T\left(\Sigma_{s}^{\text {gen }}\right)=\Sigma_{a}^{\text {gen }}$.

(2) Denote for $\vec{\kappa} \in \Sigma^{\text {gen }}$

$$
\begin{aligned}
\boldsymbol{i}(\vec{\kappa}) & :=\left|\left\{1 \leq j \leq m: \tan \left(\frac{\kappa_{j}}{2}\right)<0\right\}\right|, \\
C(\vec{\kappa}) & :=\left\{\begin{array}{ll}
1 & F_{a}(\vec{\kappa}) \leq 0 \\
0 & F_{a}(\vec{\kappa})>0
\end{array} .\right.
\end{aligned}
$$


The Neumann surplus and nodal surplus functions (introduced in Lemma 5.2 and Theorem 5.1 and satisfy, for $\vec{\kappa} \in \Sigma_{s}^{\text {gen }}$,

$$
\begin{aligned}
& \boldsymbol{\sigma}(\vec{\kappa})=\boldsymbol{i}(\vec{\kappa})-C(\vec{\kappa}), \text { and } \\
& \boldsymbol{\omega}(\vec{\kappa})=E-\boldsymbol{i}(\vec{\kappa})-C(\vec{\kappa}) .
\end{aligned}
$$

For $\vec{\kappa} \in \Sigma_{a}^{\text {gen }}, T(\vec{\kappa}) \in \Sigma_{s}^{\text {gen }}$ and

$$
\begin{aligned}
\boldsymbol{\sigma}(\vec{\kappa}) & =\boldsymbol{\sigma}(T(\vec{\kappa})), \text { and } \\
\boldsymbol{\omega}(\vec{\kappa}) & =\boldsymbol{\omega}(T(\vec{\kappa})) .
\end{aligned}
$$

Remark A.9. The $s, a$ labeling of $\Sigma_{s}^{\text {gen }}, \Sigma_{a}^{\text {gen }}$ stands for symmetric and anti-symmetric, as these parts are defined according to a certain symmetry of the eigenfunctions. It will be introduced in the proof of the Lemma.

The proof of Lemma A.8 appears after the proof of the Proposition A.5.

Proof of Proposition A.5. Define $t_{j}:=\tan \left(\frac{\kappa_{j}}{2}\right)$ so that $\vec{\kappa} \in \Sigma_{s}^{\text {gen }}$ if and only if $t_{j} \in \mathbb{R} \backslash\{0\}$ for every $j$ and the following conditions hold:

$$
\begin{aligned}
& \sum_{j=1}^{E} t_{j}=0, \text { and } \\
& \sum_{j=1}^{E} \frac{1}{t_{j}} \neq 0 .
\end{aligned}
$$

In such case $\boldsymbol{i}(\vec{\kappa})$ is the number of negative $t_{j}$ 's, and it is not hard to show that for any $1 \leq i \leq E-1$, one can construct a tuple of $t_{j}$ 's that satisfy the above conditions such that the number of negative $t_{j}$ 's is equal to $i$. Namely, there exist $\vec{\kappa} \in \Sigma_{s}^{\text {gen }}$ with $\boldsymbol{i}(\vec{\kappa})=i$ for any $1 \leq i \leq E-1=\beta$. In such case, by Lemma A.8 (2),

$$
\begin{aligned}
\boldsymbol{\sigma}(\vec{\kappa}) & =i-C(\vec{\kappa}) \in\{i, i-1\}, \\
\boldsymbol{\omega}(\vec{\kappa}) & =E-i-C(\vec{\kappa}) \in\{\beta+1-i, \beta-i\}, \text { and so } \\
\boldsymbol{\sigma}(\vec{\kappa})-\boldsymbol{\omega}(\vec{\kappa}) & =2 i-E .
\end{aligned}
$$

It follows that the image of $\boldsymbol{\sigma}-\boldsymbol{\omega}$ is $\{2-E, 4-E \ldots, E-2\}$, and that the Neumann surplus is bounded by

$$
0 \leq \boldsymbol{\omega} \leq \beta
$$

Moreover, it follows that for any $j \in\{0,1, \ldots \beta-1\}$, Image $(\boldsymbol{\omega})$ contains at least one of $j, j+1$. Same for Image $(\boldsymbol{\sigma})$. Due to the $T$ invariance the restriction of each of the above functions to $\Sigma_{a}^{\text {gen }}$ has the same image as the restriction to $\Sigma_{s}^{\text {gen }}$. This ends the proof of Proposition A.5 by the same arguments used in the proof of Proposition A.1.

As already stated, the bounds $1 \leq \sigma \leq \beta-1$ was shown in [10], but for completeness we will prove that $\boldsymbol{\sigma}(\vec{\kappa}) \neq 0$ from which the above bounds follows from symmetry arguments. Assume by contradiction that there is a point $\vec{\kappa} \in \Sigma_{s}^{\text {gen }}$ for which $\boldsymbol{\sigma}(\vec{\kappa})=0$. It follows that $\boldsymbol{i}(\vec{\kappa})=1$ and $C(\vec{\kappa})=1$. Without loss of generality assume that the negative $t_{j}$ is $t_{1}$ so that the $t_{j}$ 's satisfy

$$
\left|t_{1}\right|=\sum_{j=2}^{E} t_{j}
$$


and $C(\vec{\kappa})=1$ implies that

$$
\sum_{j=2}^{E} \frac{1}{t_{j}} \leq \frac{1}{\left|t_{1}\right|}=\frac{1}{\sum_{j=2}^{E} t_{j}} .
$$

As the $t_{j}$ 's for $j \geq 2$ are strictly positive, we get

$$
\frac{\sum_{j=2}^{E} t_{j}}{E-1} \leq \frac{1}{E-1} \frac{1}{\sum_{j=2}^{E} \frac{1}{t_{j}}}<\frac{E-1}{\sum_{j=2}^{E} \frac{1}{t_{j}}}
$$

which contradicts the means inequality.

The proof of Lemma A.8 is very similar to that of Lemma A.4 and we will therefore provide less details.

Proof of Lemma A.8. Let $\Gamma$ be a mandarin graph, and let $v_{-}$and $v_{+}$denote its two vertices. Consider $\Gamma_{\vec{\kappa}}$ for some $\vec{\kappa} \in \mathbb{T}^{E}$ with arc-length parametrization $x_{j} \in\left[-\frac{\kappa_{j}}{2}, \frac{\kappa_{j}}{2}\right]$ for every edge $e_{j}$, such that $x_{j}= \pm \frac{\kappa_{j}}{2}$ at $v_{ \pm}$. As discussed in [10], the inversion $x_{j} \mapsto-x_{j}$ for all edges simultaneously, is an isometry of $\Gamma_{\vec{\kappa}}$ and we can choose all eigenfunctions to be symmetric \anti-symmetric. In particular, define

$$
\begin{aligned}
& \Sigma_{s}^{\text {gen }}:=\left\{\vec{\kappa} \in \Sigma^{\text {gen }}: f_{\vec{\kappa}} \text { is symmetric }\right\}, \text { and } \\
& \Sigma_{a}^{\text {gen }}:=\left\{\vec{\kappa} \in \Sigma^{\text {gen }}: f_{\vec{\kappa}} \text { is anti-symmetric }\right\} .
\end{aligned}
$$

A symmetric eigenfunction $f^{(s)}$ of eigenvalue $k=1$ has the structure:

$$
\left.f^{(s)}\right|_{e_{j}}\left(x_{j}\right)=A_{j} \cos \left(x_{j}\right) .
$$

As in the proof of Lemma A.4 if $f^{(s)}\left(v_{ \pm}\right)=0$ then $\vec{\kappa} \in \boldsymbol{B}^{(2)}$, and if $f^{(s)}\left(v_{ \pm}\right) \neq 0$ then

$$
\forall j \leq E \quad \cos \left(\kappa_{j}\right) \neq 0,
$$

and the Neumann vertex condition (on each of the vertices) gives

$$
\sum_{j=1}^{E} \tan \left(\kappa_{j}\right)=F_{s}(\vec{\kappa})=0 .
$$

In such case, the outgoing derivatives of $f^{(s)}$ does not vanish if and only if

$$
\forall j \leq E \quad \sin \left(\kappa_{j}\right) \neq 0 .
$$

In particular, as in Lemma A.4 in the case of $\vec{\kappa} \notin \boldsymbol{B}^{(2)}$ there is a unique (up to scalar multiplication) construction of a $k=1$ symmetric eigenfunction with $f^{(s)}\left(v_{ \pm}\right) \neq 0$ is and only if $F_{s}(\vec{\kappa})=0$. Moreover, if the eigenfunction is generic then (A.45) and (A.47) implies that $\vec{\kappa} \notin \boldsymbol{B}^{(1)}$.

An anti-symmetric eigenfunction $f^{(a)}$ of eigenvalue $k=1$ has the structure:

$$
\left.f^{(a)}\right|_{e_{j}}\left(x_{j}\right)=B_{j} \sin \left(x_{j}\right) .
$$

A similar argument would show that in the case of $\vec{\kappa} \notin \boldsymbol{B}^{(2)}$ there is a unique (up to scalar multiplication) construction of a $k=1$ anti-symmetric eigenfunction with $f^{(a)}\left(v_{ \pm}\right) \neq 0$ is and only if $F_{a}(\vec{\kappa})=0$. Moreover, if the eigenfunction is generic then (A.45) and A.47) implies that $\vec{\kappa} \notin \boldsymbol{B}^{(1)}$. 
We may conclude that,

$$
\begin{aligned}
& \Sigma_{s}^{\text {gen }}=\left\{\vec{\kappa} \in \mathbb{T}^{E} \backslash \boldsymbol{B}^{(1)}: F_{s}(\vec{\kappa})=0, \text { and } F_{a}(\vec{\kappa}) \neq 0\right\}, \text { and } \\
& \Sigma_{a}^{\text {gen }}=\left\{\vec{\kappa} \in \mathbb{T}^{E} \backslash \boldsymbol{B}^{(1)}: F_{s}(\vec{\kappa}) \neq 0, \text { and } F_{a}(\vec{\kappa})=0\right\} .
\end{aligned}
$$

Observe that $\left(\begin{array}{c}\cos \left(\frac{\kappa+\pi}{2}\right) \\ \sin \left(\frac{\kappa+\pi}{2}\right)\end{array}\right)=\left(\begin{array}{c}-\sin \left(\frac{\kappa}{2}\right) \\ \cos \left(\frac{\kappa}{2}\right)\end{array}\right)$, and therefore $\boldsymbol{B}^{(1)}$ is invariant to $T$ and $F_{s} \circ T=$ $-F_{a}$. It follows that

$$
T\left(\Sigma_{s}^{\text {gen }}\right)=\Sigma_{a}^{\text {gen }} .
$$

This proves Lemma A.8 (1). To prove Lemma A.8 $(2)$, consider $i(\vec{\kappa}):=\left|\left\{j \leq E: \tan \left(\frac{\kappa_{j}}{2}\right)<0\right\}\right|$ and let $\vec{\kappa} \in \Sigma_{s}^{\text {gen }}$ so that $f_{\vec{\kappa}}$ is symmetric and generic. Using A.44 we derive the nodal and Neumann counts:

$$
\begin{aligned}
& \phi\left(f_{\vec{\kappa}}\right)=2\left|\left\{j \leq E: \frac{\kappa_{j}}{2}>\frac{\pi}{2}\right\}\right|=2 i(\vec{\kappa}), \text { and } \\
& \xi\left(f_{\vec{\kappa}}\right)=E .
\end{aligned}
$$

Similarly, for $\vec{\kappa} \in \Sigma_{a}^{\text {gen }}$, using A.48,

$$
\begin{aligned}
& \phi\left(f_{\vec{\kappa}}\right)=E, \text { and } \\
& \xi\left(f_{\vec{\kappa}}\right)=2\left|\left\{j \leq E: \frac{\kappa_{j}}{2}>\frac{\pi}{2}\right\}\right|=2 \boldsymbol{i}(\vec{\kappa}) .
\end{aligned}
$$

As in the proof of Lemma A.4 (2), we define a "good" set:

$$
M:=\left\{\vec{\kappa} \in \Sigma^{\text {gen }}: \forall t \in(0,1] t \vec{\kappa} \notin \boldsymbol{B} \cup \Sigma^{\text {sing }}\right\},
$$

such that the spectral position for $\vec{\kappa} \in M$ is given by

$$
N(\vec{\kappa})=\left|\left\{t \in(0,1]: F_{s}(t \vec{\kappa})=0\right\}\right|+\left|\left\{t \in(0,1]: F_{a}(t \vec{\kappa})=0\right\}\right| .
$$

For $\vec{\kappa} \in M$ and $t \in(0,1)$ the function $t \mapsto F_{a}(t \vec{\kappa})$ is continuous, and decreasing from infinity and so

$$
\left|\left\{t \in(0,1]: F_{a}(t \vec{\kappa})=0\right\}\right|=\left\{\begin{array}{ll}
1 & F_{a}(\vec{\kappa}) \leq 0 \\
0 & F_{a}(\vec{\kappa})>0
\end{array}=C(\vec{\kappa})\right.
$$

For $\vec{\kappa} \in M$, the function $t \mapsto F_{s}(t \vec{\kappa})$ is increasing and has interlacing poles and zeros as in the proof of Lemma A.1 and using the same argument we get:

$$
\begin{gathered}
\left|\left\{t \in(0,1]: F_{s}(t \vec{\kappa})=0\right\}\right|=\left|\left\{j \leq E: \tan \left(\frac{\kappa_{j}}{2}\right)<0\right\}\right|-1+ \begin{cases}1 & F_{s}(\vec{\kappa}) \geq 0 \\
0 & F_{s}(\vec{\kappa})<0\end{cases} \\
=\boldsymbol{i}(\vec{\kappa})-1+C(T(\vec{\kappa})),
\end{gathered}
$$

so that for any $\vec{\kappa} \in M$,

$$
N(\vec{\kappa})=\boldsymbol{i}(\vec{\kappa})-1+C(\vec{\kappa})+C(T(\vec{\kappa}))
$$

Observe that $C(\vec{\kappa}) \equiv 1$ on $\Sigma_{a}^{\text {gen }}$ and so $C(T(\vec{\kappa})) \equiv 1$ on $\Sigma_{s}^{\text {gen }}$. Subtracting $N(\vec{\kappa})$ from A.50 and (A.51), for any $\vec{\kappa} \in M \cap \Sigma_{s}^{\text {gen }}$, gives

$$
\begin{aligned}
& \boldsymbol{\sigma}(\vec{\kappa})=\boldsymbol{i}(\vec{\kappa})-C(\vec{\kappa}), \text { and } \\
& \boldsymbol{\omega}(\vec{\kappa})=E-\boldsymbol{i}(\vec{\kappa})-C(\vec{\kappa}) .
\end{aligned}
$$


Similarly for $\vec{\kappa} \in M \cap \Sigma_{a}^{\text {gen }}$,

$$
\begin{aligned}
& \boldsymbol{\sigma}(\vec{\kappa})=E-\boldsymbol{i}(\vec{\kappa})-C(T(\vec{\kappa}))=\boldsymbol{i}(T(\vec{\kappa}))-C(T(\vec{\kappa}))=\boldsymbol{\sigma}(T(\vec{\kappa})), \\
& \boldsymbol{\omega}(\vec{\kappa})=\boldsymbol{i}(\vec{\kappa})-C(T(\vec{\kappa}))=E-\boldsymbol{i}(T(\vec{\kappa}))-C(T(\vec{\kappa}))=\boldsymbol{\omega}(T(\vec{\kappa})) .
\end{aligned}
$$

The same argument as in the proof of Lemma A.1 shows that $M$ is dense in $\Sigma^{\text {gen }}$ and so the above holds for all $\vec{\kappa} \in \Sigma^{\text {gen }}$.

A.3. Trees. As a particular case of Proposition A.1, the Neumann surplus of a star graph is bounded and gets all integer values between -1 to $1-|\partial \Gamma|$. This result can be generalized to tree graphs using the following lemma:

Lemma A.10. Let $\Gamma^{(1)}$ and $\Gamma^{(2)}$ be tree graphs with edge lengths $\vec{l}^{(1)}$ and $\vec{l}^{(2)}$, and let $\Gamma$ be the tree graph obtained by gluing the two graphs at two boundary vertices $v_{1} \in \partial \Gamma^{(1)}$ and $v_{2} \in \partial \Gamma^{(2)}$. Let $f^{(1)}$ and $f^{(2)}$ be generic eigenfunctions of $\Gamma^{(1)}$ and $\Gamma^{(2)}$ with the same eigenvalue $k$ and define the function $f$ on $\Gamma$ by:

$$
\begin{aligned}
\left.f\right|_{\Gamma^{(1)}} & =\frac{1}{f^{(1)}\left(v_{1}\right)} f^{(1)}, \\
\left.f\right|_{\Gamma^{(2)}} & =\frac{1}{f^{(2)}\left(v_{2}\right)} f^{(2)} .
\end{aligned}
$$

Then $f$ is a generic eigenfunction of $\Gamma$ with Neumann surplus:

$$
\omega(f)=\omega\left(\left.f\right|_{\Gamma^{(1)}}\right)+\omega\left(\left.f\right|_{\Gamma^{(2)}}\right)+1 .
$$

Proof. First we show that $f$ is an eigenfunction of $\Gamma$. Let $x_{0}$ be the point in $\Gamma$ which is identified with both $v_{1}$ and $v_{2}$. As $\operatorname{deg}\left(x_{0}\right)=2$ we will consider it as an interior point $x_{0} \in \Gamma \backslash \partial \Gamma$ and denote the edge containing $x_{0}$ by $e_{0}$. By construction, the Neumann vertex conditions are satisfied on all vertices of $\Gamma$, and the restrictions of $f$ on every edge $e \neq e_{0}$ satisfies the ODE, $\left.f\right|_{e} ^{\prime \prime}=-\left.k^{2} f\right|_{e}$. It is also clear that the latter ODE holds for $f$ restricted to $e_{0} \backslash\left\{x_{0}\right\}$, and we only need to show that both $f$ and $f^{\prime}$ are continuous at $x_{0}$ in order to conclude that $\left.f\right|_{e_{0}} ^{\prime \prime}=-\left.k^{2} f\right|_{e_{0}}$. The normalization of the two parts of $f$ was chosen such that, $\left.f\right|_{\Gamma^{(1)}}\left(x_{0}\right)=\frac{1}{f^{(1)}\left(v_{1}\right)} f^{(1)}\left(v_{1}\right)=1$, and in the same way, $\left.f\right|_{\Gamma^{(2)}}\left(x_{0}\right)=1$. The two directional derivatives at $x_{0}$ are

$$
\begin{aligned}
& \left.f\right|_{\Gamma^{(1)}} ^{\prime}\left(x_{0}\right) \propto \partial_{e_{1}} f^{(1)}\left(v_{1}\right)=0, \\
& \left.f\right|_{\Gamma^{(2)}} ^{\prime}\left(x_{0}\right) \propto \partial_{e_{2}} f^{(2)}\left(v_{2}\right)=0 .
\end{aligned}
$$

This proves that $f$ is an eigenfunction of $\Gamma$. By its construction, it satisfies both conditions (2) and (3) of the genericity in Definition 1.3, and is therefore generic (see Remark 1.4). Since $f$ is generic, we may consider its nodal count $\phi(f)$ and Neumann count $\xi(f)$. By construction, $\phi(f)=\phi\left(f^{(1)}\right)+\phi\left(f^{(2)}\right)$ and since $x_{0}$ is an interior point on which $f^{\prime}\left(x_{0}\right)=0$ then $\xi(f)=\xi\left(f^{(1)}\right)+\xi\left(f^{(2)}\right)+1$. Since $\Gamma, \Gamma^{(1)}$ and $\Gamma^{(2)}$ are trees then $\sigma(f), \sigma\left(f^{(1)}\right)$ and $\sigma\left(f^{(2)}\right)$ are zero (see 3.2$)$. Therefore,

$$
\omega\left(f^{(1)}\right)=\omega\left(f^{(1)}\right)-\sigma\left(f^{(1)}\right)=\xi\left(f^{(1)}\right)-\phi\left(f^{(1)}\right),
$$

and in the same way

$$
\begin{aligned}
\omega\left(f^{(2)}\right) & =\xi\left(f^{(2)}\right)-\phi\left(f^{(2)}\right), \text { and } \\
\omega(f) & =\xi(f)-\phi(f) .
\end{aligned}
$$


Therefore,

$$
\begin{aligned}
\omega(f) & =\xi(f)-\phi(f) \\
& =\left(\xi\left(f^{(1)}\right)-\phi\left(f^{(1)}\right)\right)+\left(\xi\left(f^{(2)}\right)-\phi\left(f^{(2)}\right)\right)+1 \\
& =\omega\left(f^{(1)}\right)+\omega\left(f^{(2)}\right)+1 .
\end{aligned}
$$

Proposition A.11. If $\Gamma$ is a tree graph then its Neumann surplus is bounded by

$$
-|\partial \Gamma|+1 \leq \omega \leq-1 .
$$

Moreover, if $\Gamma$ has rationally independent edge lengths then its Neumann surplus distribution satisfies:

$$
\forall j \in\{-|\partial \Gamma|+1,-|\partial \Gamma|+2 \ldots,-1\} \quad P(\omega=j)>0 .
$$

Proof. Theorem 2.2 (1) ensures that the Neumann surplus of a tree is bounded between $-|\partial \Gamma|+1$ and -1 . According to Theorem 2.2 (2b), Theorem 4.8 and Lemma 5.2 , given a fixed value $j$, if there exist some $\vec{l} \in \mathbb{R}_{+}^{E}$ and some generic eigenfunction $f$ of $\Gamma_{\vec{l}}$ such that $\omega(f)=j$, then for any rationally independent $\vec{l}$ the Neumann surplus distribution has $P(\omega=j)>0$.

We will prove that for any tree $\Gamma$ and any $j \in\{-|\partial \Gamma|+1, \ldots-1\}$, there exist some $\vec{l} \in \mathbb{R}_{+}^{E}$ and some generic eigenfunction $f$ of $\Gamma_{\vec{l}}$ for which $\omega(f)=j$. The proof is done by induction on the number of interior vertices $V_{\text {in }}:=|\mathcal{V} \backslash \partial \Gamma|$, where the case $V_{\text {in }}=1$ is a star graph, for which the statement is true by Lemma A.1.

Let $m>1$ and assume that the statement holds for any tree with $V_{i n}<m$. Let $\Gamma$ be a tree with $V_{i n}=m$, then there is an edge $e_{0}$ which is not a tail. For $x_{0} \in e_{0}$ which is an interior point, $\Gamma \backslash\left\{x_{0}\right\}$ has two connected components, $\Gamma^{(1)}$ and $\Gamma^{(2)}$, which are both trees. Clearly, both $\Gamma^{(1)}$ and $\Gamma^{(2)}$ have less then $m$ interior vertices. By the assumption, the statement holds for both $\Gamma^{(1)}$ and $\Gamma^{(2)}$. That is, for each $i \in\{1,2\}$, for any $j_{i} \in\left\{-\left|\partial \Gamma_{i}\right|+1, \ldots-1\right\}$, there exist $\vec{l}^{(i)}$ and a generic eigenfunction $f^{(i)}$ of $\Gamma_{\vec{l}(i)}^{(i)}$ with Neumann surplus $\omega\left(f^{(i)}\right)=j_{i}$. We may scale $\vec{l}^{(i)}$ such that $f^{(i)}$ has eigenvalue $k=1$. Consider $\Gamma$ with edge lengths according to the gluing of $\Gamma_{\vec{l}^{(1)}}^{(1)}$ and $\Gamma_{\vec{l}^{(2)}}^{(2)}$ at $x_{0}$. By Lemma A.10, $\Gamma$ has a generic eigenfunction $f$ with

$$
\omega(f)=j_{1}+j_{2}+1 .
$$

By construction $|\partial \Gamma|=\left|\partial \Gamma_{1}\right|+\left|\partial \Gamma_{2}\right|-2$ and so $j:=j_{1}+j_{2}+1$ ranges over all values between

$$
-|\partial \Gamma|+1=\left(-\left|\partial \Gamma_{1}\right|+1\right)+\left(-\left|\partial \Gamma_{2}\right|+1\right)+1 \leq j \leq-1-1+1=-1 .
$$

By induction, the statement is true for any choice of $V_{i n}$. This proves the Lemma.

\section{Appendix B. Proofs of Lemma 4.2 and Lemma 4.12}

Proof of Lemma 4.2. We start by providing a basic tool in the spectral analysis of metric graphs. Let $\Gamma$ be a standard graph with $E:=|\mathcal{E}|$ edges. For every $\vec{\kappa} \in \mathbb{T}^{E}$ we abuse notation and denote by $\mathrm{e}^{\mathrm{i} \vec{\kappa}} \in U(2 E)$ the following unitary diagonal matrix

$$
\mathrm{e}^{\mathrm{i} \vec{\kappa}}:=\operatorname{diag}\left(\mathrm{e}^{\mathrm{i} \kappa_{1}}, \mathrm{e}^{\mathrm{i} \kappa_{1}}, \mathrm{e}^{\mathrm{i} \kappa_{2}}, \mathrm{e}^{\mathrm{i} \kappa_{2}}, \ldots, e^{\mathrm{i} \kappa_{E}}, e^{\mathrm{i} \kappa_{E}}\right)
$$


such that every exponent $\mathrm{e}^{\mathrm{i} \kappa_{j}}$ appears twice. Given a choice of edge lengths $\vec{l}$ and an eigenvalue $k$ it is convenient to denote the matrix $\mathrm{e}^{\mathrm{i} \vec{\kappa}}$ for $\vec{\kappa}=[k \vec{l}]$ by $\mathrm{e}^{\mathrm{i} k \vec{l}}$. There exists an orthogonal matrix $S \in O(2 E)$, uniquely determined by the connectivity of $\Gamma$, which is independent of the edge lengths and has the following property. A positive $k>0$ is an eigenvalue of $\Gamma$ if and only if $\operatorname{ker}\left(\mathbb{I}-\mathrm{e}^{\mathrm{i} k \vec{l}} S\right)$ is nontrivial [5, Section 3.2]. This relation is a consequence of an isomorphism between $\operatorname{ker}\left(\mathbb{I}-\mathrm{e}^{\mathrm{i} k \vec{l}} S\right)$ and the $k$-eigenspace of $\Gamma$. Explicitly, this isomorphism sends $\boldsymbol{a} \in \operatorname{ker}\left(\mathbb{I}-\mathrm{e}^{\mathrm{i} k \vec{l}} S\right)$ to an eigenfunction $f$ of the eigenvalue $k$ such that the restriction to every edge $e \in \mathcal{E}$, is given by

$$
\left.f\right|_{e}(x)=a_{e} \mathrm{e}^{\mathrm{i} k x} e^{-i k l_{e}}+a_{\hat{e}} \mathrm{e}^{-\mathrm{i} k x},
$$

where $x \in\left[0, l_{e}\right]$ is an arc-length parametrization of the edge $e \in \mathcal{E}$, and $a_{e}, a_{\hat{e}}$ are the two entries of the vector $\boldsymbol{a}$ which correspond to edge $e$ (according to the indexing of $\mathrm{e}^{\mathrm{i} \vec{k}}$ which was introduced above). As an implication we get for each $\vec{l}$ an isomorphism between the $k$-eigenspace of $\Gamma_{\vec{l}}$ and the 1-eigenspace of $\Gamma_{\vec{k}}$, where $\vec{\kappa}=[k \vec{l}]$. This, together with 4.1 proves part (1) of the Lemma.

The above argument also implies that $\vec{\kappa} \in \Sigma^{\text {reg }}$ if and only if $\operatorname{dim} \operatorname{ker}\left(1-e^{i \vec{\kappa}} S\right)=1$. For any such $\vec{\kappa}$ value, the adjugate matrix $\operatorname{adj}\left(\mathbb{I}-\mathrm{e}^{\mathrm{i} \vec{\kappa}} S\right)$ is a rank one matrix. Hence, there exists a non-trivial $\boldsymbol{a} \in \operatorname{ker}\left(\mathbb{I}-\mathrm{e}^{\mathrm{i} \vec{k}} S\right)$ such that $\operatorname{adj}\left(\mathbb{I}-\mathrm{e}^{\mathrm{i} \vec{k}} S\right)=\boldsymbol{a} \boldsymbol{a}^{*}$. We use this $\boldsymbol{a}$ to introduce a function $f_{\vec{k}}$ by $(\bar{B} .2)$. This provides a family of canonical eigenfunctions $\left\{f_{\vec{k}}\right\}_{\vec{\kappa} \in \Sigma^{\mathrm{reg}}}$, as in the statement of the lemma. We should note that these functions are not uniquely determined. This is since the vectors $\boldsymbol{a}$ above are determined only up to a multiplication by some unitary factor $c \in U(1)$; indeed $\operatorname{adj}\left(\mathbb{I}-\mathrm{e}^{\mathrm{i} \vec{\kappa}} S\right)=\boldsymbol{a} \boldsymbol{a}^{*}$ is invariant with respect to such a multiplication of $\boldsymbol{a}$, and the factor might depend on $\vec{\kappa}$. Next, we show that this family of canonical eigenfunctions $\left\{f_{\vec{k}}\right\}_{\vec{k} \in \Sigma^{\text {reg }}}$ indeed satisfy the requirements in the lemma.

First, note that the arguments above already prove parts (2a) and (3) of the lemma (the $c \in \mathbb{C}$ factor appears in part (3) since the canonical eigenfunction, $f_{\vec{k}}$, is not necessarily real). We proceed to prove part (2b) of the lemma.

Let $v \in \mathcal{V}$ and $e \in \mathcal{E}_{v}$ with arc-length parametrization $x \in\left[0, l_{e}\right]$ such that $v$ is at $x=0$. By (B.2) we get that for each $\vec{\kappa} \in \Sigma^{\text {reg }}$,

$$
\begin{aligned}
f_{\vec{\kappa}}(v) & =\left.f_{\vec{\kappa}}\right|_{e}(0)=a_{e} \mathrm{e}^{-\mathrm{i} \kappa_{e}}+a_{\hat{e}} \\
\partial_{e} f_{\vec{\kappa}}(v) & =\left.f_{\vec{k}}\right|_{e} ^{\prime}(0)=\mathrm{i}\left(a_{e} \mathrm{e}^{-\mathrm{i} \kappa_{e}}-a_{\hat{e}}\right) .
\end{aligned}
$$

Observe that each term $f_{\vec{k}}(v)$ or $\partial_{e} f_{\vec{k}}(v)$ is linear in the $\boldsymbol{a}$ entries with coefficients which are taken from $\left(\mathrm{e}^{-\mathrm{i} \kappa_{1}}, \ldots, \mathrm{e}^{-\mathrm{i} \kappa_{E}}\right)$. Therefore, each product of the form $f_{\vec{k}}(u) \overline{f_{\vec{k}}(v)}$ or $f_{\vec{k}}(u) \overline{\partial_{e} f_{\vec{k}}(v)}$ is linear in the $\boldsymbol{a a}^{*}$ entries with coefficients which are trigonometric polynomials (in $\left.\left(\kappa_{1}, \ldots, \kappa_{E}\right)\right)$. But, the entries of $\boldsymbol{a} \boldsymbol{a}^{*}=\operatorname{adj}\left(\mathbb{I}-\mathrm{e}^{\mathrm{i} \vec{k}} S\right)$ are minors of $\mathbb{I}-\mathrm{e}^{\mathrm{i} \vec{k}} S$ and so themselves trigonometric polynomials in $\left(\kappa_{1}, \ldots, \kappa_{E}\right)$. Hence, $f_{\vec{k}}(u) \overline{f_{\vec{k}}(v)}$ is a trigonometric polynomial in $\left(\kappa_{1}, \ldots, \kappa_{E}\right)$, which we denote by $p_{u, v}(\vec{\kappa})$; and $f_{\vec{\kappa}}(u) \overline{\partial_{e} f_{\vec{\kappa}}(v)}$ is a trigonometric polynomial in $\left(\kappa_{1}, \ldots, \kappa_{E}\right)$, which we denote by $q_{u, v, e}(\vec{\kappa})$. This proves the relations $(4.2)$ and (4.3) in the lemma and it is left to show that $p_{u, v}$ and $q_{u, v, e}$ are actually real trigonometric polynomials. To do this, we redefine $p_{u, v}$ and $q_{u, v, e}$ to be equal to their real parts and argue that (4.2) and (4.3) are still valid after such a modification. Indeed, all products $f_{\vec{k}}(u) \overline{f_{\vec{k}}(v)}$ and $f_{\vec{k}}(u) \overline{\partial_{e} f_{\vec{k}}(v)}$ are real since $f_{\vec{k}}$ is an eigenfunction of $\Gamma$ corresponding a simple eigenvalue and as such it is real up to a global multiplicative factor. 
Proof of Lemma 4.12. We begin by referring to the proof of Lemma 4.2 above where it was argued that

$$
\vec{\kappa} \in \Sigma \Leftrightarrow \operatorname{dim} \operatorname{ker}\left(\mathbb{I}-\mathrm{e}^{\mathrm{i} \vec{\kappa}} S\right)>0
$$

and

$$
\vec{\kappa} \in \Sigma^{\mathrm{reg}} \Leftrightarrow \operatorname{dim} \operatorname{ker}\left(\mathbb{I}-\mathrm{e}^{\mathrm{i} \vec{\kappa}} S\right)=1 .
$$

Combining this with the relation

$$
\forall \vec{\kappa} \in \mathbb{T}^{E}, \quad \mathbb{I}-\mathrm{e}^{\mathrm{i} \mathcal{I}(\vec{\kappa})} S=\overline{\left(\mathbb{I}-\mathrm{e}^{\mathrm{i} \vec{\kappa}} S\right)},
$$

gives

$$
\vec{\kappa} \in \Sigma \Leftrightarrow \mathcal{I}(\vec{\kappa}) \in \Sigma \quad \text { and } \quad \vec{\kappa} \in \Sigma^{\mathrm{reg}} \Leftrightarrow \mathcal{I}(\vec{\kappa}) \in \Sigma^{\mathrm{reg}},
$$

and proves that $\Sigma$ and $\Sigma^{\text {reg }}$ are each invariant under $\mathcal{I}$. We may further deduce that $\operatorname{adj}\left(\mathbb{I}-\mathrm{e}^{\mathrm{i} \mathcal{I}(\vec{\kappa})} S\right)=\overline{\operatorname{adj}\left(\mathbb{I}-\mathrm{e}^{\mathrm{i} \vec{\kappa}} S\right)}$ and so if $\boldsymbol{a}$ is a vector for which $\boldsymbol{a} \boldsymbol{a}^{*}=\operatorname{adj}\left(\mathbb{I}-\mathrm{e}^{\mathrm{i} \vec{\kappa}} S\right)$ then $\overline{\boldsymbol{a}}$ is such that $\overline{\boldsymbol{a \boldsymbol { a }}}^{*}=\operatorname{adj}\left(\mathbb{I}-\mathrm{e}^{\mathrm{i} \mathcal{I}(\vec{\kappa})} S\right)$. These vectors define $f_{\vec{\kappa}}$ and $f_{\mathcal{I}(\vec{\kappa})}$ by $($ B.3 and (B.4) and so we get

$$
\begin{aligned}
\forall v & \in \mathcal{V}, \quad f_{\mathcal{I}(\vec{\kappa})}(v)=\overline{a_{e}} \mathrm{e}^{-\mathrm{i}[\mathcal{I}(\vec{\kappa})]_{e}}+\overline{a_{\hat{e}}}=\overline{a_{e} \mathrm{e}^{-\mathrm{i} \kappa_{e}}+a_{\hat{e}}} \quad \text { and } \\
\forall v \in \mathcal{V}, \forall e \in \mathcal{E}_{v} \quad \partial_{e} f_{\mathcal{I}(\vec{\kappa})}(v) & =\mathrm{i}\left(\overline{a_{e}} \mathrm{e}^{-\mathrm{i}[\mathcal{I}(\vec{\kappa})]_{e}}-\overline{a_{\hat{e}}}\right)=-\overline{\mathrm{i}\left(a_{e} \mathrm{e}^{-\mathrm{i} \kappa_{e}}-a_{\hat{e}}\right)} .
\end{aligned}
$$

Comparing the RHS above with $(\mathrm{B} .3)$ and $(\mathrm{B} .4)$ shows

$$
\forall v \in \mathcal{V}, \forall e \in \mathcal{E}_{v} \quad f_{\mathcal{I}(\vec{\kappa})}(v)=c \overline{f_{\vec{\kappa}}(v)} \quad \text { and } \quad \partial_{e} f_{\mathcal{I}(\vec{\kappa})}(v)=-\bar{c} \overline{\partial_{e} f_{\vec{\kappa}}(v)},
$$

where $c \in U(1)$ is a multiplicative factor which expresses a degree of freedom in determining $\boldsymbol{a}$ (alternatively $f_{\vec{k}}$ ) and $c$ might depend on $\vec{\kappa}$; see also in proof of Lemma 4.2 . From (B.11) together with the definition of $\Sigma^{\text {gen }}$, (4.6) we get that $\Sigma^{\text {gen }}$ is invariant under $\mathcal{I}$, which finishes the proof of part (1) of the Lemma.

To continue proving the second part of the lemma, we note that (B.11) implies in particular that all products $f_{\vec{\kappa}}(u) \overline{f_{\vec{\kappa}}(v)}$ are $\mathcal{I}$ invariant and all products $f_{\vec{\kappa}}(u) \overline{\partial_{e} f_{\vec{\kappa}}(v)}$ are $\mathcal{I}$ anti-symmetric. As a conclusion we get that the real trigonometric polynomials $p_{u, v}$ and $q_{u, v, e}$ that are defined in the proof of Lemma 4.2 satisfy

$$
\begin{aligned}
\forall \vec{\kappa} \in \Sigma^{\text {reg }} p_{u, v}(\mathcal{I}(\vec{\kappa})) & =p_{u, v}(\vec{\kappa}), \text { and } \\
\forall \vec{\kappa} \in \Sigma^{\text {reg }} q_{u, v, e}(\mathcal{I}(\vec{\kappa})) & =-q_{u, v, e}(\vec{\kappa}) .
\end{aligned}
$$

Note that these are almost the required relations (1), (3). But (B.12) holds for $\vec{\kappa} \in \Sigma^{\mathrm{reg}}$, whereas $(1),(3)$ are stated for all $\vec{\kappa} \in \mathbb{T}^{E}$. Let us abuse notation once again and redefine $p_{u, v}$ as $\frac{p_{u, v}+p_{u, v} \mathcal{I}}{2}$ and $q_{u, v, e}$ as $\frac{q_{u, v, e}-q_{u, v, e} \circ \mathcal{I}}{2}$. This ensures that $p_{u, v}$ and $q_{u, v, e}$ are $\mathcal{I}$ symmetric and anti-symmetric respectively, while being real trigonometric polynomials whose restrictions are equal to the products $f_{\vec{\kappa}}(u) \overline{f_{\vec{\kappa}}(v)}$ and $f_{\vec{\kappa}}(u) \overline{\partial_{e} f_{\vec{\kappa}}(u)}$ for any $\vec{\kappa} \in \Sigma^{r e g}$. To show that $\Sigma^{\text {gen }}$ is $\mathcal{I}$ invariant, recall that $\Sigma^{\text {gen }}$ is the set of $\vec{\kappa} \in \Sigma^{\text {reg }}$ for which $f_{\vec{\kappa}}$ is generic. Definition 1.3 for genericity has three conditions, where condition (1) holds if and only if $\vec{\kappa} \in \Sigma^{\text {reg }}$. The other two conditions, (2) and (3), hold if and only if $p_{u, u}(\vec{\kappa}) \neq 0$ and $q_{v, v, e}(\vec{\kappa}) \neq 0$ for all $u \in \mathcal{V}, v \in \mathcal{V} \backslash \partial \Gamma$ and $e \in \mathcal{E}_{v}$. As $\Sigma^{\text {reg }}$ is $\mathcal{I}$ invariant and $p_{u, u}$ and $q_{v, v, e}$ are $\mathcal{I}$ symmetric $\backslash$ anti-symmetric, then $f_{\mathcal{I}(\vec{\kappa})}$ is generic if and only if $f_{\vec{\kappa}}$ is and therefore $\Sigma^{\text {gen }}$ is $\mathcal{I}$ invariant.

The third part of our Lemma is stated and proved in [5, lem. 3.13]. 


\section{Appendix C. An analogue of Proposition 2.7 for nodal domains}

This appendix provides bounds on the wavelength capacity and the Neumann count of nodal domains.

Proposition C.1. Let $\Gamma$ be a standard graph with minimal edge length $L_{\min }$. Let $k^{2}$ be an eigenvalue of $\Gamma$ which satisfies $k>\frac{\pi}{L_{\min }}$. Let $\Omega_{D}$ be a nodal domain of a generic eigenfunction $f$ which corresponds to $k$. Denote the total length of $\Omega_{D}$ by $\left|\Omega_{D}\right|$, its wavelength capacity by $\rho\left(\Omega_{D}\right):=\frac{\left|\Omega_{D}\right| k}{\pi}$, and its Neumann count by $\xi\left(\Omega_{D}\right):=\xi\left(\left.f\right|_{\Omega_{D}}\right)$. The following bounds hold

$$
\begin{aligned}
1 & \leq \xi\left(\Omega_{D}\right) \leq\left|\partial \Omega_{D}\right|-1 \\
1 \leq \frac{1}{2}\left(\xi\left(\Omega_{D}\right)+1\right) & \leq \rho\left(\Omega_{D}\right) \leq \frac{1}{2}\left(\xi\left(\Omega_{D}\right)+\left|\partial \Omega_{D}\right|-1\right) \leq\left|\partial \Omega_{D}\right|-1
\end{aligned}
$$

Remark C.2. The spectral position of a Neumann domain is equal to its nodal count. Hence, the bounds (2.7) in Proposition 2.7 may be perceived as the bounds on the nodal count of a Neumann domain. Therefore, we consider the bounds in (C.1) of the proposition above as the analogous bounds. Furthermore, there is no interest in bounds for the spectral position of a nodal domain, as it trivially equals to 1 (see Section 1.5).

Proof. We start by noting that the condition $k>\frac{\pi}{L_{\min }}$ guarantees that $\Omega_{D}$ is either a star graph or an interval (see also Lemma 3.1). If $\Omega_{D}$ is an interval the bounds follow trivially as $\xi\left(\Omega_{D}\right)=1$ and $\rho\left(\Omega_{D}\right)=1$. We proceed by assuming that $\Omega_{D}$ is a star graph. From here, the main argument in the proof is a map from star graphs which are nodal domains (such as $\Omega_{D}$ ) to star graphs which are Neumann domains, as described next. Denote the edge lengths of $\Omega_{D}$ by $\left\{l_{j}\right\}_{j=1}^{\left|\partial \Omega_{D}\right|}$. We may write $\left.f\right|_{\Omega_{D}}$ on every edge $e_{j}$ of $\Omega_{D}$ as

$$
\left.f\right|_{e_{j}}(x)=A_{j} \sin \left(k\left(x-l_{j}\right)\right) \quad x \in\left[0, l_{j}\right],
$$

where $x=0$ at the central vertex and $x=l_{j}$ at the boundary vertex. The absence of nodal points in the interior of $\Omega_{D}$ together with the genericity of $f$ imply that $k l_{j} \in\left(0, \frac{\pi}{2}\right) \cup\left(\frac{\pi}{2}, \pi\right)$ for each $j$. We use this to construct an auxiliary star graph, $\Omega_{N}$, which has the same number of edges, $\left|\partial \Omega_{N}\right|=\left|\partial \Omega_{D}\right|$ and its edge lengths are given by

$$
\forall 1 \leq j \leq\left|\partial \Omega_{N}\right|, \quad \tilde{l}_{j}= \begin{cases}l_{j}-\frac{\pi}{2 k} & k l_{j} \in\left(\frac{\pi}{2}, \pi\right) \\ l_{j}+\frac{\pi}{2 k} & k l_{j} \in\left(0, \frac{\pi}{2}\right) .\end{cases}
$$

Define a function $\tilde{f}$ on $\Omega_{N}$ by

$$
\left.\tilde{f}\right|_{\tilde{e}_{j}}(x)=A_{j} \sin \left(k\left(x-l_{j}\right)\right) \quad x \in\left[0, \tilde{l}_{j}\right],
$$

for any edge $\tilde{e}_{j}$ of $\Omega_{N}$, where $A_{j}$ and $l_{j}$ are the same as in (C.3). This construction guarantees $\left.\tilde{f}^{\prime}\right|_{e_{j}}\left(\tilde{l}_{j}\right)=A_{j} \sin \left( \pm \frac{\pi}{2}\right)=0$ and so $\tilde{f}$ satisfies Neumann conditions at the boundary vertices of $\Omega_{N}$. In addition, $\tilde{f}$ and $f$ share the same value and derivatives at the corresponding central vertex (of $\Omega_{N}$ and of $\Omega_{D}$ ). Hence, $\tilde{f}$ satisfies Neumann vertex conditions at the central vertex of $\Omega_{N}$. We conclude that $\tilde{f}$ is an eigenfunction of $\Omega_{N}$ with eigenvalue $k$.

Since $f$ and $\tilde{f}$ share the same value and derivatives at the central vertex, and $f$ is generic, then $\tilde{f}$ satisfies both conditions $(2)$ and $(3)$ of the genericity in Definition 1.3 . In addition, $k$ is a simple eigenvalue of $\Omega_{N}$ by an argument similar to the one which was given in the proof of Lemma 3.1] (this argument is based on [20, Corollary 3.1.9] and on $\Omega_{N}$ being 
a tree). Therefore, $\tilde{f}$ is generic. By the construction, $\tilde{f}$ has no interior Neumann points, so that $\Omega_{N}$ is a single Neumann domain of the generic eigenfunction $\tilde{f}$. In particular, the bounds on $N\left(\Omega_{N}\right)$ and $\rho\left(\Omega_{N}\right)$ in Proposition 2.7 apply to it. To finish the proof we just need to relate $N\left(\Omega_{N}\right)$ and $\rho\left(\Omega_{N}\right)$ to $\xi\left(\Omega_{D}\right)$ and $\rho\left(\Omega_{D}\right)$ and apply those bounds. The needed relations follow from a simple calculations based on (C.3) and (C.5):

$$
\phi\left(\left.\tilde{f}\right|_{\Omega_{N}}\right)+\xi\left(\Omega_{D}\right)=\left|\partial \Omega_{D}\right|
$$

and

$$
\rho\left(\Omega_{N}\right)=\frac{1}{\pi} \sum_{j=1}^{\left|\partial \Omega_{N}\right|} \tilde{l}_{j}=\frac{1}{\pi}\left(\sum_{j=1}^{\left|\partial \Omega_{D}\right|}\left(l_{j}+\frac{\pi}{2}\right)-\pi \xi\left(\left.f\right|_{\Omega_{D}}\right)\right)=\rho\left(\Omega_{D}\right)+\frac{\left|\partial \Omega_{D}\right|}{2}-\xi\left(\Omega_{D}\right) .
$$

Using the relations above with $\phi\left(\left.\tilde{f}\right|_{\Omega_{N}}\right)=N\left(\Omega_{N}\right)$ (see 3.11$)$ ) and applying the bounds on $N\left(\Omega_{N}\right)$ and $\rho\left(\Omega_{N}\right)$ in Proposition 2.7 yields all the desired bounds in (C.1) and (C.2).

\section{REFERENCES}

[1] O. AL-OBeID, On the number of the constant sign zones of the eigenfunctions of a dirichlet problem on a network (graph), tech. rep., Voronezh: Voronezh State University, 1992. in Russian, deposited in VINITI 13.04.93, N 938 - B 93. - 8 p. 1.8

[2] L. Alon, Generic eigenfunctions of quantum graphs, In preperation. 1.6, 3, 4.3, 6

[3] L. Alon, Quantum graphs - Generic eigenfunctions and their nodal count and Neumann count statistics, PhD thesis, Mathamtics Department, Technion - Israel Institute of Technology, 2020. 4.1. $4.2,4.3,4.1,6,6.2,7$

[4] L. Alon, R. BAnd, And G. Berkolaiko, On a universal limit conjecture for the nodal count statistics of quantum graphs, In preperation. 1, 8

[5] _ Nodal Statistics On Quantum Graphs, Communications in Mathematical Physics, (2018). 1. 1.6, 3, 2.1, 2.1, 4.1, 4.2, 4.1, 4.3, 4.1, 6, 4.3, 5.1, 5.1, 6.4, 6.2, 7, 8, B, B

[6] L. Alon, R. BAnd, M. Bersudsky, AND S. EgGER, Neumann domains on graphs and manifolds, in Analysis and Geometry on Graphs and Manifolds, vol. 461 of London Math. Soc. Lecture Note Ser., Cambridge Univ. Press, 2020, pp. 203-249. 1, 1.5, 8, 12, 8

[7] R. BAnd, The nodal count $\{0,1,2,3, \ldots\}$ implies the graph is a tree, Philos. Trans. R. Soc. Lond. A, 372 (2014), pp. 20120504, 24. preprint arXiv:1212.6710. 1. 5.1, 8

[8] R. Band, G. Berkolaiko, H. Raz, and U. Smilansky, The number of nodal domains on quantum graphs as a stability index of graph partitions, Commun. Math. Phys., 311 (2012), pp. 815-838. 1.2

[9] R. Band, G. Berkolaiko, and U. Smilansky, Dynamics of nodal points and the nodal count on a family of quantum graphs, Annales Henri Poincare, 13 (2012), pp. 145-184. 1.4

[10] R. Band, G. Berkolaiko, and T. Weyand, Anomalous nodal count and singularities in the dispersion relation of honeycomb graphs, J. Math. Phys., 56 (2015), p. 122111. 1. A.6, A.2, A.2

[11] R. BAnd, G. Cox, AND S. EGGER, Defining the spectral position of a Neumann domain, arXiv:2009.14564. 1, 1.5, 8

$[12]$ _ Spectral properties of Neumann domains via the Dirichlet-to-Neumann operator. In preparation. $1.5,8$

[13] R. Band, S. Egger, and A. TaYlor, The spectral position of Neumann domains on the torus, J. Geom. Anal., (2020).https://doi.org/10.1007/s12220-020-00444-9.1. 1.5. 8

[14] R. Band and D. Fajman, Topological properties of Neumann domains, Ann. Henri Poincaré, 17 (2016), pp. 2379-2407. 1, 1.5, 8

[15] R. BAnd And S. Gnutzmann, Quantum graphs via exercises, in Spectral theory and applications, vol. 720 of Contemp. Math., Amer. Math. Soc., Providence, RI, 2018, pp. 187-203. 1.2

[16] R. Band, T. Shapira, And U. Smilansky, Nodal domains on isospectral quantum graphs: the resolution of isospectrality?, J. Phys. A, 39 (2006), pp. 13999-14014. 1. 8 
[17] F. Barra and P. Gaspard, On the level spacing distribution in quantum graphs, J. Statist. Phys., 101 (2000), pp. 283-319. 4.8, 4.9

[18] G. Berkolaiko, A lower bound for nodal count on discrete and metric graphs, Comm. Math. Phys., 278 (2008), pp. 803-819. 1, $1.4,2$

[19] G. Berkolaiko, An elementary introduction to quantum graphs, in Geometric and computational spectral theory, vol. 700 of Contemp. Math., Amer. Math. Soc., Providence, RI, 2017, pp. 41-72. 1.2

[20] G. Berkolaiko and P. Kuchment, Introduction to Quantum Graphs, vol. 186 of Mathematical Surveys and Monographs, AMS, 2013. 1.2, 1.2, 1.4, C

[21] G. Berkolaiko And W. Liu, Simplicity of eigenvalues and non-vanishing of eigenfunctions of a quantum graph, J. Math. Anal. Appl., 445 (2017), pp. 803-818. preprint arXiv:1601.06225. 3

[22] G. Berkolaiko and T. Weyand, Stability of eigenvalues of quantum graphs with respect to magnetic perturbation and the nodal count of the eigenfunctions, Philos. Trans. R. Soc. Lond. Ser. A Math. Phys. Eng. Sci., 372 (2014), pp. 20120522, 17. 1

[23] G. Berkolaiko And B. Winn, Relationship between scattering matrix and spectrum of quantum graphs, Trans. Amer. Math. Soc., 362 (2010), pp. 6261-6277.4.8, 4.9

[24] J. Brüning and D. Fajman, On the nodal count for flat tori, Communications in Mathematical Physics, 313 (2012), pp. 791-813. 8

[25] Y. Colin DE Verdière, Semi-classical measures on quantum graphs and the Gauß map of the determinant manifold, Annales Henri Poincaré, 16 (2015), pp. 347-364. also arXiv:1311.5449.4.1. $4.2,4.2,4.8,4.9,7$

[26] R. Courant, Ein allgemeiner Satz zur Theorie der Eigenfunktione selbstadjungierter Differentialausdrücke, Nach. Ges. Wiss. Göttingen Math.-Phys. Kl., (1923), pp. 81-84. 1 ]

[27] M. Einsiedler AND T. WARD, Ergodic theory, Springer, 2013. 4.4 4.5

[28] Y. Elon, S. Gnutzmann, C. Joas, and U. Smilansky, Geometric characterization of nodal domains: the area-to-perimeter ratio, J. Phys. A: Math. Theor., 40 (2007), p. 2689. 1.5

[29] L. Friedlander, Extremal properties of eigenvalues for a metric graph, Ann. Inst. Fourier (Grenoble), 55 (2005), pp. 199-211. 2.8, 3. 5.1

[30] S. Gnutzmann AND U. Smilansky, Quantum graphs: Applications to quantum chaos and universal spectral statistics, Adv. Phys., 55 (2006), pp. 527-625. 1.2

[31] S. Gnutzmann, U. Smilansky, and N. SondergaARD, Resolving isospectral 'drums' by counting nodal domains., J. Phys. A, 38(41) (2005), pp. 8921-8933. 8

[32] S. Gnutzmann, U. Smilansky, and J. Weber, Nodal counting on quantum graphs, Waves Random Media, 14 (2004), pp. S61-S73. 1. 1.4

[33] M. Hofmann, J. B. Kennedy, D. Mugnolo, and M. PlÃCEmer, Asymptotics and estimates for spectral minimal partitions of metric graphs, 2020. 2

[34] J. S. JuUl AND C. H. Joyner, Isospectral discrete and quantum graphs with the same flip counts and nodal counts, Journal of Physics A: Mathematical and Theoretical, 51 (2018), p. 245101. 1, 8

[35] J. Kennedy, P. Kurasov, C. Léna, and D. Mugnolo, A theory of spectral partitions of metric graphs, arXiv: Spectral Theory, (2020). 2

[36] T. Kottos and U. Smilansky, Quantum chaos on graphs, Phys. Rev. Lett., 79 (1997), pp. $4794-$ 4797. 2.3

[37] _ Periodic orbit theory and spectral statistics for quantum graphs, Ann. Physics, 274 (1999), pp. $76-124.2 .3$

[38] S. G. Krantz and H. R. Parks, A primer of real analytic functions, Birkhäuser Advanced Texts: Basler Lehrbücher. [Birkhäuser Advanced Texts: Basel Textbooks], Birkhäuser Boston, Inc., Boston, MA, second ed., 2002.5.1

[39] R. B. McDonald and S. A. Fulling, Neumann nodal domains, Philos. Trans. R. Soc. Lond. Ser. A Math. Phys. Eng. Sci., 372 (2014), pp. 20120505, 6. 1

[40] B. Mityagin, The zero set of a real analytic function, arXiv:1512.07276, (2015). 7

[41] I. ORen AND R. BAND, Isospectral graphs with identical nodal counts, J. Phys. A, 45 (2012), p. 135203. preprint arXiv:1110.0158. 8

[42] A. Pleijel, Remarks on courant's nodal line theorem, Communications on Pure and Applied Mathematics, 9 (1956), pp. 543-550. 1

[43] Y. V. PokornyĬ, V. L. Pryadiev, And A. AL'-ObeId, On the oscillation of the spectrum of a boundary value problem on a graph, Mat. Zametki, 60 (1996), pp. 468-470. 1.8 
[44] S. P. Ponomarev, Submersions and preimages of sets of measure zero, Siberian Mathematical Journal, 28 (1987), pp. 153-163.7

[45] P. Schapotschnikow, Eigenvalue and nodal properties on quantum graph trees, Waves in Random and Complex Media, 16 (2006), pp. 167-78. 1. 8

[46] C. Sturm, Mémoire sur les équations différentielles linéaires du second ordre, J. Math. Pures Appl., 1 (1836), pp. 106-186. 1

[47] S. ZelDitch, Eigenfunctions and nodal sets, Surveys in Differential Geometry, 18 (2013), pp. 237308. 1

${ }^{1}$ Lior Alon, School of Mathematics, Institute for Advanced Study, Princeton, NEW JERSEY 08540, USA.

${ }^{2}$ Ram Band, Department of Mathematics, Technion-Israel Institute of TechNOLOGY, HAifa 32000, IsRAEL. 